\title{
Mathematical Formulation Requirements and Specifications for the Process Models
}

\author{
November 9, 2010
}

ASCEM-HPC-10-01-1

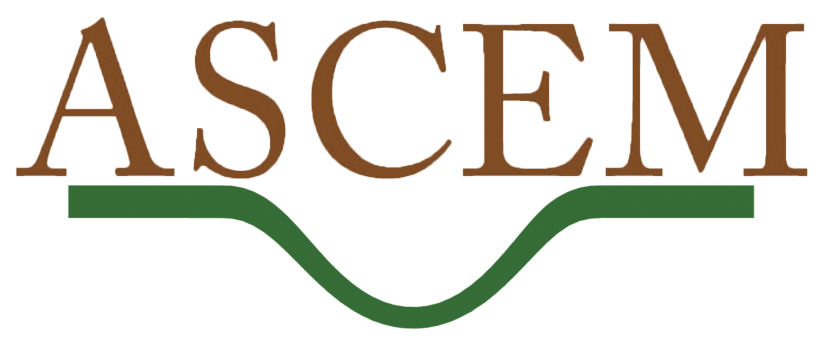

\section{United States Department of Energy}

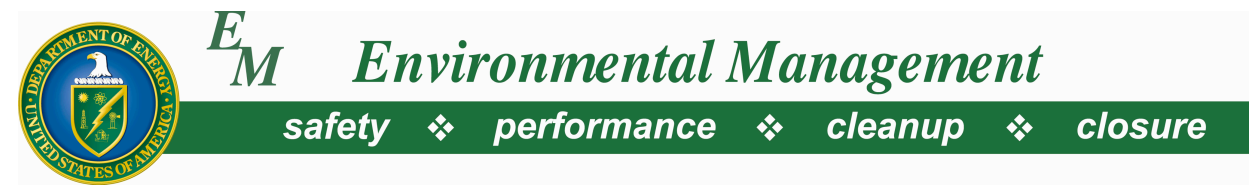

C. Steefel, LBNL

P. Lichtner, LANL J. Bell, LBNL

G. Zyvoloski, LANL

D. Moulton, LANL

T. Wolery, LLNL

G. Moridis, LBNL

B. Andre, LBNL

G. Pau, LBNL

D. Bacon, PNNL

S. Yabusaki, PNNL

L. Zheng, LBNL

K. Lipnikov, LANL

N. Spycher, LBNL

E. Sonnenthal, LBNL

J. Davis, LBNL

J. Meza, LBNL

LBNL affiliates were partially supported by U.S. DOE and LBNL under Contract No. DE-AC02-05CH11231. 


\section{DISCLAIMER}

This work was prepared under an agreement with and funded by the U.S. Government. Neither the U.S. Government or its employees, nor any of its contractors, subcontractors or their employees, makes any express or implied:

1. warranty or assumes any legal liability for the accuracy, completeness, or for the use or results of such use of any information, product, or process disclosed; or

2. representation that such use or results of such use would not infringe privately owned rights; or

3. endorsement or recommendation of any specifically identified commercial product, process, or service.

Any views and opinions of authors expressed in this work do not necessarily state or reflect those of the United States Government, or its contractors, or subcontractors.

\section{Printed in the United States of America}

Prepared for U.S. Department of Energy 


\section{Authorization:}

Dr. Paul Dixon, Los Alamos National Laboratory

Date

Multi-Lab ASCEM Program Manager

Dr. Juan Meza, Lawrence Berkeley National Laboratory

ASCEM Technical Integration Manager

Dr. J. David Moulton, Los Alamos National Laboratory

ASCEM Multi-Process HPC Simulator Thrust Lead

Dr. Carl I. Steefel, Lawrence Berkeley National Laboratory ASCEM Multi-Process HPC Simulator Deputy Thrust Lead

Date

Date

\section{Concurrence:}

Mark Williamson, ASCEM Program Manager

Date

EM-32 Groundwater \& Soil Remediation

Kurt Gerdes, EM-32

Date

Office Director for Groundwater \& Soil Remediation 


\section{Table of Contents}

1 Introduction to Process Models Requirements $\quad 7$

1.1 ASCEM Overview . . . . . . . . . . . . . . . . 7

1.2 Purpose and Scope of this Document . . . . . . . . . . . . . . 7

1.3 Links to Other Thrust Areas . . . . . . . . . . . . . . . . . 8

1.4 Organization and Layout of this Document . . . . . . . . . . . . . . . . . 9

1.5 Prioritization and Selection of Process Models . . . . . . . . . . . . . . . 9

2 Overview of Process Models $\quad 11$

2.1 Continuum Hypothesis . . . . . . . . . . . . . . . . . . 11

2.2 Fluid Description . . . . . . . . . . . . . . . . . 11

2.3 Governing Equations . . . . . . . . . . . . . . . . . . . 12

2.4 Phase Behavior . . . . . . . . . . . . . . . . . . . 14

2.5 Reactive Transport . . . . . . . . . . . . . . . . . 16

2.6 Fractured and Highly Heterogeneous Media . . . . . . . . . . . . . . . . . . 19

3 Flow Processes $\quad 21$

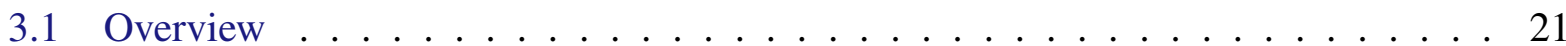

3.2 Assumptions and Applicability for Flow Equations . . . . . . . . . . . . . . 21

3.3 General Formulation for Multiphase Flow . . . . . . . . . . . . . . . . . . . . 21

3.4 Richards' Equation . . . . . . . . . . . . . . . . . . . . . . 24

3.5 Single-Phase Flow . . . . . . . . . . . . . . . . . . . . . 25

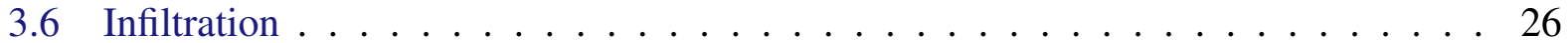

3.7 Data Requirements for Flow . . . . . . . . . . . . . . . . . . . . . . . . . . . 29

3.8 Reaction-Induced Porosity and Permeability Change . . . . . . . . . . . . . 30

4 Transport Processes $\quad 33$

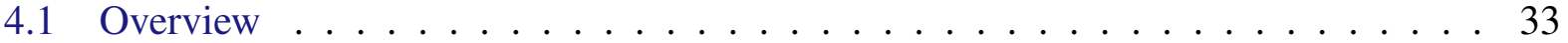

4.2 Process Model Equations for Transport . . . . . . . . . . . . . . . . . . 33

4.3 Boundary Conditions, Sources and Sinks . . . . . . . . . . . . . . . . . . . . 34

4.4 Advective Transport . . . . . . . . . . . . . . . . . . . . . . . 34

4.5 Dispersive Transport . . . . . . . . . . . . . . . . . . 35

4.6 Diffusive Transport . . . . . . . . . . . . . . . . . . . 36

5 Biogeochemical Reaction Processes $\quad 42$

5.1 Aqueous Complexation . . . . . . . . . . . . . . . . . . . 42 
5.2 Aqueous Activity Coefficients . . . . . . . . . . . . . . . . . . 44

5.3 Sorption . . . . . . . . . . . . . . . . . . . . . . 69

5.4 Mineral Precipitation and Dissolution . . . . . . . . . . . . . . . . 79

5.5 Microbially-Mediated Reactions _ . . . . . . . . . . . . . . . . . 86

6 Colloid Transport Processes $\quad 90$

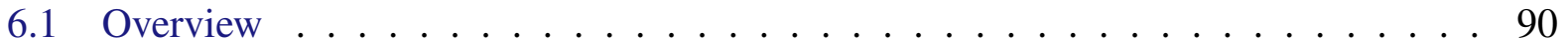

6.2 Process Model Equations . . . . . . . . . . . . . . . . . . . . 96

7 Thermal Processes $\quad 101$

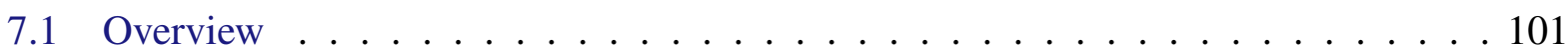

7.2 Process Model Equations . . . . . . . . . . . . . . . . . . . . . . 101

7.3 Data Needs . . . . . . . . . . . . . . . . . . . . . . . . . 101

7.4 Boundary Conditions, Sources and Sinks . . . . . . . . . . . . . . . . 101

7.5 Coupling Considerations . . . . . . . . . . . . . . . . . 102

8 Geomechanical Processes $\quad 103$

8.1 Overview . . . . . . . . . . . . . . . . . . 103

8.2 Assumptions and Applicability . . . . . . . . . . . . . . . . . . . . 103

8.3 THM Formulation . . . . . . . . . . . . . . . . . . . . . . . 103

8.4 Single-Phase Biot Model . . . . . . . . . . . . . . . . . . . . . . . . . 104

8.5 Boundary Conditions . . . . . . . . . . . . . . . . . . 105

9 Source Terms 106

9.1 Cementitious Source Terms . . . . . . . . . . . . . . . . . . . . 106

9.2 Glass Waste Forms ． . . . . . . . . . . . . . . . . . . . . . 106

9.3 High-Level Radioactive Waste Tanks as Source Terms . . . . . . . . . . . . . . 111

$\begin{array}{lr}\text { A Nomenclature } & 113\end{array}$

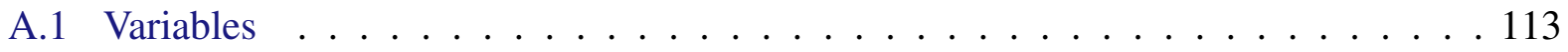




\section{List of Tables}

1 An initial list of process models proposed for the Phase I demonstration is listed along with their corresponding subsection. . . . . . . . . . . . . . 10

2 Key Pitzer interaction coefficients. . . . . . . . . . . . . . . . . 55

3 Key Pitzer parameters other than interaction coefficients. . . . . . . . . . . . . 56

4 Chebyshev polynomial coefficients for computing $J(x)$ and $J^{\prime}(x)$ (Harvie, 1981) . 60

5 List of Latin variables. Unitless variables are indicated by — . . . . . . . . . . 113

6 List of Greek variables. Unitless variables are indicated by — . . . . . . . . . . 116

7 List of variables used in the Pitzer, UNIQUAC and NEA SIT models, Section 5.2 . 117

\section{List of Figures}

1 Tortuous diffusion paths in porous media (Steefel and Maher, 2009) . . . . . . . . 38

2 Schematic of the TLM model from Gonçalvès et al. (2007) . . . . . . . . . . . . . . 80

3 A conceptual image of the colloid classes I-III is presented along with their origins. 93

4 General schematic of the stages of glass-water reaction . . . . . . . . . . 107 


\section{Introduction to Process Models Requirements}

\subsection{ASCEM Overview}

The Advanced Simulation Capability for Environmental Management (ASCEM) is intended to be a state-of-the-art scientific tool and approach for understanding and predicting contaminant fate and transport in natural and engineered systems. The ASCEM program is aimed at addressing critical EM program needs to better understand and quantify flow and contaminant transport behavior in complex geological systems. It will also address the long-term performance of engineered components including cementitious materials in nuclear waste disposal facilities, in order to reduce uncertainties and risks associated with DOE EM's environmental cleanup and closure activities. Building upon national capabilities developed from decades of Research and Development in subsurface geosciences, computational and computer science, modeling and applied mathematics, and environmental remediation, the ASCEM initiative will develop an integrated, open-source, highperformance computer modeling system for multiphase, multicomponent, multiscale subsurface flow and contaminant transport. This integrated modeling system will incorporate capabilities for predicting releases from various waste forms, identifying exposure pathways and performing dose calculations, and conducting systematic uncertainty quantification. The ASCEM approach will be demonstrated on selected sites, and then applied to support the next generation of performance assessments of nuclear waste disposal and facility decommissioning across the EM complex.

The Multi-Process High Performance Computing (HPC) Simulator is one of three thrust areas in ASCEM. The other two are the Platform and Integrated Toolsets (dubbed the Platform) and Site Applications. The primary objective of the HPC Simulator is to provide a flexible and extensible computational engine to simulate the coupled processes and flow scenarios described by the conceptual models developed using the ASCEM Platform. The graded and iterative approach to assessments naturally generates a suite of conceptual models that span a range of process complexity, potentially coupling hydrological, biogeochemical, geomechanical, and thermal processes. The Platform will use ensembles of these simulations to quantify the associated uncertainty, sensitivity, and risk. The Process Models task within the HPC Simulator focuses on the mathematical descriptions of the relevant physical processes.

\subsection{Purpose and Scope of this Document}

At the highest level of the HPC Simulator design is a set of process models that mathematically represent the physical, chemical, and biological phenomena controlling contaminant release into, and transport in, the subsurface. The objective of this requirements document is to provide a catalogue of process models, along with their detailed mathematical formulation, for potential implementation in the HPC Simulator. This concise mathematical description and accompanying analysis, provides critical information for requirements and design of both the HPC Core Framework (Task 1.1.2.2) and the HPC Toolsets (Task 1.1.2.3). This effort will also leverage ongoing efforts elsewhere in the Department of Energy, including the Cementitious Barrier Project (CBP) funded also by Environmental Management through EM-31.

It is important to note that with its focus on mathematical descriptions for a catalogue of process 
models, this requirements document is significantly different from a traditional Software Requirements Specification (SRS) document. Hence, this document does not follow the IEEE Std 8301998 template, and instead uses a process category based layout that is summarized in Section 1.4. Moreover, this mathematical focus serves multiple audiences:

1. This document provides guidance to the developers engaged in designing and implementing the HPC Core Framework and HPC Toolsets. To meet their needs, sufficient detail for each process model is provided in the form of a background discussion, supporting equations, and references to relevant literature. The process models that are currently targeted for implementation in the Phase 1 demonstration are listed in Table 1.

2. The document is also intended for "domain scientists" whose primary interest is in the processes themselves. The presentation is intended to justify the choice of process models and their mathematical detail.

3. Finally, the document is also intended for end users engaged in individual site applications.

Over time this document will evolve into a comprehensive graded presentation of models, from complex to simple, under a general mathematical framework for each process category. The prioritization and selection of process models from this catalogue for implementation is discussed in Section 1.5. Evolution of the list of processes is inevitable, and the modular design of the HPC Simulator will easily accommodate the addition of new process implementations.

\subsection{Links to Other Thrust Areas}

The set of requirements detailed in this document have a clear connection to the other two thrust areas in ASCEM. In particular, many of the process model requirements follow from recommendations made from end users as captured in the User Suggestions for ASCEM Requirements Documents (Section 5, pp. 18-19) (Seitz et al., 2010). These requirements include the need for multiphase flow and transport, reactive transport, and both groundwater flow. Surface water flow, an important process at some contaminated sites, will be added as needed at a later date, but is currently beyond the scope of the present effort. In addition, there is a need for modeling the degradation of engineered barriers, including, for example, covers, liners, cementitious materials and waste forms. Other aspects that were deemed desirable include the modeling of radionuclides, source releases, and fractured media. All of these needs have been recognized and addressed in some form within this document.

Similarly, there are several areas where the process models will need to interface with the Platform and Integrated Toolsets Thrust Area. The Platform Thrust Area is tasked with developing the tools necessary to set up the conceptual models. These models represent the subsurface flow and reactive transport processes described in this document and will include 1) the geologic setting or framework, 2) the physical, geochemical, and biological processes and their interactions, and 3) the scenarios to which the models are applied. An end user wishing to perform a simulation will have to specify features such as the process models, the initial and boundary conditions, and the material properties. These features will have to be consistent with and fully integrated with the existing capabilities of the process models. For example, for each process model that is available, 
there will be an associated set of constitutive models from which a user can select. In turn, each process model will have a set of specifications that will dictate the parameter requirements that are specific to the particular model and associated constitutive relations. Because of these links, there needs to be close ties between the development of the process models and the tools being developed by the Platform Thrust area so that the final simulation package can be reliable, robust, and easy to use.

\subsection{Organization and Layout of this Document}

The remainder of the Mathematical Formulation Requirements and Specifcations for the Process Models document is organized as follows. It presents an overview of the mathematical framework that is used to model subsurface flow and reactive transport (Section 2). This overview is followed by a series of sections focusing on individual process categories. These include a presentation of Flow Processes in Section 3, Transport Processes in Section 4, Biogeochemical Reaction Processes in Section 5, Colloid Transport Processes in Section 6, Thermal Processes in Section 7 , Geomechanical Processes in Section 8, and Source Terms, in Section 9. Each of these process categories may present multiple process models. For example, biogeochemistry includes individual process models for sorption, mineral precipitation-dissolution, microbially-mediated reactions, colloid generation, etc. In addition, models of differing fidelity can be accommodated by the HPC code so, for example, several models for sorption (e.g., classical Kd, multicomponent-multisite ion exchange, non-electrostatic surface complexation, electrostatic surface complexation) are included.

The difficult question of prioritizing and ultimately selecting process models for implementation in any given year is addressed in the following subsection (Section 1.5). Finally, the notational conventions and variables are summarized in Section A.1.

\subsection{Prioritization and Selection of Process Models}

Within each section that focuses on a particular process category (e.g., flow, transport), subsections contain the details of specific process models that may be implemented in the HPC Simulator. However, the inclusion of a process model in this document does not guarantee it will be implemented in the HPC Simulator in the near term. A combination of many factors will be considered in the prioritization of process models and selection for implementation in any given year. For example, these factors include, the relative importance of the process models to various EM sites and the availability of required data for EM sites of interest. These types of information are gathered and organized by the Site Application Thrust. In the first years of the ASCEM project, prioritization must also consider the overhead of initiating development of the supporting HPC Core Framework (i.e., supporting infrastructure), as well as the design and initial development of the HPC Toolsets (i.e., the fundamental algorithmic building blocks).

Given these constraints, preliminary discussions involving all three thrusts have led to a list of proposed process models for the first year's development and the Phase I demonstration. This list of proposed process models is presented in Table 1.

Finally, it is important to note that the set of process models described in this document is by no 
Table 1: An initial list of process models proposed for the Phase I demonstration is listed along with their corresponding subsection.

\begin{tabular}{lll} 
Process Category & Process Model & Section \\
\hline \hline Flow & Single-phase & 3.5 \\
Flow & Richards & 3.4 \\
\hline Transport & Non-reactive, single component & 4 \\
Transport & Reactive, multicomponent & 4 \\
\hline Biogeochemical Reactions & Sorption & 5.3 \\
Biogeochemical Reactions & Precipitation/Dissolution & 5.4 \\
\hline \hline
\end{tabular}

means exhaustive. Process models will be added based on the prioritization of specific EM needs during periodic reviews and updates of this document. 


\section{Overview of Process Models}

There are many formulations of the process models for contaminant transport that one could present. Here we will start with the equations for conservation of mass and energy in the presence of chemical reactions for a multiphase-multicomponent system. This leads to the basic flow equations describing non-isothermal, multiphase, multicomponent flows in heterogeneous porous media. A short description of phase behavior follows, which explains the relationship between the composition of the phases and the flow equations. An introduction to some of the chemical concepts needed concludes this section.

There are other components describing the physical processes that must be included, including more detailed descriptions of transport, chemical processes, transport of colloids, geothermal, mechanical, and source terms. In addition, within flow there are many processes that must be taken into account, the major one being infiltration from various sources including surface water, evaporation and transpiration. All of these process models are described in greater detail in subsequent chapters.

\subsection{Continuum Hypothesis}

A rock mass consisting of aggregates of mineral grains and pore spaces or voids is referred to as a porous medium. An actual porous medium is a highly heterogeneous structure containing physical discontinuities marked by the boundaries of pore walls which separate the solid framework from the void space. Although it is possible in principle at least, to describe this system at the microscale of individual pores, such a description rapidly becomes a hopeless task as the size of the system increases and many pore volumes become involved. It is therefore necessary to approximate the system by a more manageable one. One quantitative description of the transport of fluids and their interaction with rocks is based on a mathematical idealization of the real physical system referred to as a continuum. In this theory the actual discrete physical system, consisting of aggregates of mineral grains, interstitial pore spaces, and fractures, is replaced by a continuous system in which physical variables describing the system vary continuously in space. Allowance is made for the possibility of a discrete set of surfaces across which discontinuous changes in physical properties may occur. In this fictitious representation of the real physical system solids and fluids coexist simultaneously at each point in space.

\subsection{Fluid Description}

A material body may be grouped into different regions having homogeneous properties at some scale called phases. A phase may be considered a homogeneous mixture of its constituents. Jump discontinuities in various properties generally occur at the boundaries of phases. For example, a rock is considered an aggregate of different minerals each of which constitute a separate phase. For a system in thermodynamic equilibrium a minimal set of independent components are used to describe the system that may or may not correspond to the actual constituents in the system.

To start, we will denote the porosity of the medium by $\phi$. We assume that the pore space is filed with one or more phases. The saturation of each phase, $s_{\alpha}$ denotes the fraction of the pore 
volume occupied by that phase. The fluids are composed of $N$ chemical components (or lumped components) that can appear in one or more phases. The components therefore are conserved quantities that need to be tracked potentially across multiple phases. The phases themselves are not conserved. We denote by $n_{k \alpha}$ the number of moles of component $k$ in phase $\alpha$ per unit pore volume, or, in vector form $\boldsymbol{n}_{\alpha}$. Thus, $\sum_{\alpha} \boldsymbol{n}_{\boldsymbol{\alpha}} \equiv \boldsymbol{n}$ is the vector of total moles of each component per unit pore volume in the multiphase fluid system with elements $n_{k}$. We note that the system can be analogously defined by the composition of the fluid in terms of mass per unit pore volume, $M_{k \alpha}$ or, in vector form $M_{\alpha}$. We can also define $n_{\alpha}=\sum_{k} n_{k \alpha}$ and $M_{\alpha}=\sum_{k} M_{k \alpha}$ to be the total moles and mass, respectively, per unit pore volume in phase $\alpha$. With these definitions the mass density of phase $\alpha$ is $\rho_{\alpha}=M_{\alpha} / s_{\alpha}$.

From these basic definitions we can define several other useful ways to characterize the composition of the phases, some of which are typically used in subsurface groundwater systems. Mass fractions are defined as

$$
Y_{k \alpha}=\frac{M_{k \alpha}}{M_{\alpha}}
$$

mole fractions are defined as

$$
X_{k \alpha}=\frac{n_{k \alpha}}{n_{\alpha}}
$$

molarity is defined as

$$
C_{k \alpha}=\frac{n_{k \alpha}}{s_{\alpha}}
$$

and molality is defined as

$$
m_{k \alpha}=\frac{n_{k \alpha}}{M_{0 \alpha}}
$$

where $M_{0 \alpha}$ denotes the mass of solvent in phase $\alpha$ per unit pore volume. Another useful measure of concentration is the mass density $\rho_{i \alpha}$ defined as

$$
\begin{aligned}
\rho_{k \alpha} & =\frac{M_{k \alpha}}{s_{\alpha}}=\frac{M_{\alpha}}{s_{\alpha}} \frac{M_{k \alpha}}{M_{\alpha}} \\
& =\rho_{\alpha} Y_{k \alpha}=W_{k} C_{k \alpha}
\end{aligned}
$$

where $W_{k}$ is the molecular weight of component $k$. Or a molar density, $\rho_{k \alpha}^{\prime}$, can be defined

$$
\rho_{k \alpha}^{\prime}=\frac{n_{k \alpha}}{s_{\alpha}}=\rho_{\alpha}^{\prime} X_{k \alpha}
$$

With these definitions,

$$
\begin{gathered}
n_{k \alpha}=\rho_{\alpha}^{\prime} X_{k \alpha} s_{\alpha}, \\
n_{k}=\sum_{\alpha} \rho_{\alpha}^{\prime} X_{k \alpha} s_{\alpha} .
\end{gathered}
$$

\subsection{Governing Equations}

The flow is governed by the equations of mass, momentum, and energy conservation. 


\subsubsection{Conservation of Momentum}

The conservation of momentum in groundwater systems is typically given by Darcy's law, which describes the volumetric flow rate, $\boldsymbol{q}_{\alpha}$, of each phase in terms of the phase pressure, $p_{\alpha}$ by

$$
\boldsymbol{q}_{\alpha}=-\frac{\mathbf{k} k_{r \alpha}}{\mu_{\alpha}}\left(\nabla p_{\alpha}-\rho_{\alpha} \boldsymbol{g}\right)
$$

where $\mathbf{k}$ is the permeability (tensor) of the medium, $k_{r, \alpha}$ is the relative permeability, which expresses the modification of the flow rate by multiphase effects, $\mu_{\alpha}$ and $\rho_{\alpha}$ are the phase viscosity and density, respectively, and $\boldsymbol{g}$ is the gravitational force. The pressure in each phase is related to a reference pressure, $p$, typically taken to be the least wetting phase by a capillary pressure, $p_{c, \alpha}=p_{\alpha}-p$, which is a function of saturation.

\subsubsection{Conservation of Mass}

Molar (or mass) conservation for each component is given by

$$
\frac{\partial(\phi \boldsymbol{n})}{\partial t}+\nabla \cdot \sum_{\alpha} \frac{\boldsymbol{n}_{\boldsymbol{\alpha}}}{s_{\alpha}} \boldsymbol{q}_{\alpha}=\nabla \cdot \boldsymbol{D}+\boldsymbol{R}+\boldsymbol{Q},
$$

or equivalently, making use of (2.3), in notation perhaps more commonly found in groundwater hydrology

$$
\frac{\partial\left(\phi \sum_{\alpha} s_{\alpha} \boldsymbol{C}_{\boldsymbol{\alpha}}\right)}{\partial t}+\nabla \cdot \sum_{\alpha} \boldsymbol{C}_{\boldsymbol{\alpha}} \boldsymbol{q}_{\alpha}=\boldsymbol{\nabla} \cdot \boldsymbol{D}+\boldsymbol{R}+\boldsymbol{Q} .
$$

We note that the component conservation equation are often written in terms of mole fractions using Eqns. (2.8) and (2.9). Here $\boldsymbol{D}$ are diffusive terms that include multiphase molecular diffusion and dispersion, $\boldsymbol{R}$ are reaction terms and $\boldsymbol{Q}$ are source terms. Both the diffusion / dispersion, reaction terms and source terms can be quite complex. Their particular forms are discussed elsewhere.

\subsubsection{Conservation of Energy}

For non-isothermal systems it is necessary to include the energy conservation in the system of equations. The overall energy balance must include energy in the solid phase. If we assume that the porous medium and the fluids are in thermal equilibrium, the total energy balance is of the form

$$
\frac{\partial H_{\mathrm{t}}}{\partial t}+\nabla \cdot \sum_{\alpha} \frac{\boldsymbol{q}_{\alpha}}{s_{\alpha}} \boldsymbol{n}_{\boldsymbol{\alpha}}^{T} \boldsymbol{h}_{\boldsymbol{\alpha}}=\nabla \cdot Q_{T}+R_{H}+\frac{\partial \sum_{\alpha} p_{\alpha} u_{\alpha}}{\partial t}+\nabla \cdot \sum_{\alpha} \boldsymbol{q}_{\alpha} \cdot \nabla p_{\alpha}
$$

where

$$
H_{\mathrm{t}}=(1-\phi) \rho_{r} H_{r}+\phi \sum_{\alpha} \boldsymbol{n}_{\boldsymbol{\alpha}}^{T} \boldsymbol{h}_{\boldsymbol{\alpha}}
$$

is the total enthalpy of the system, $u_{\alpha}$ is the internal energy of phase $\alpha, \boldsymbol{h}_{\boldsymbol{\alpha}}$ are the partial molar phase enthalpies, $H_{r}$ is the enthalpy of the medium and $\rho_{r}$ is the density of the medium. Here $Q_{T}$ represents other energy transport processes such as thermal conduction, radiation, or dispersive 
heat transfer, and $R_{H}$ represents energy release from reactions and external heating. This represents a form of the energy equation in which the solid and the fluid phases are in thermal equilibrium. If they are not, the fluid and solid portions of the enthalpy become distinct variables and $R_{H}$ will contain relaxation terms that equilibrate the two temperatures.

In most cases, the term on the right hand side of Eqn. (2.13),

$$
\frac{\partial \sum_{\alpha} p_{\alpha} u_{\alpha}}{\partial t}+\nabla \cdot \sum_{\alpha} \boldsymbol{q}_{\alpha} \cdot \nabla p_{\alpha}
$$

can be omitted from the system. Omitting this term implicitly assumes that the change in phase pressures is slow so that these terms can be ignored. It is worth noting that many people formulate the energy equation without this term and replace total enthalpy by total internal energy. Formulations based on internal energy make a similar assumption by dropping a term of the form

$$
\sum_{\alpha} p_{\alpha} \nabla \cdot \boldsymbol{q}_{\alpha}
$$

from the energy equation.

\subsection{Phase Behavior}

The component conservation equations express the change in total moles of each of the component due to advection, diffusion, and chemical reactions. Since components are being transported in phases, it is necessary to know the composition of the phases before we can solve the flow equations. This decomposition is referred to as the phase behavior of the system. For the nonisothermal system, the phase behavior is determined by saying that the equilibrium state of the mixture occurs at the point of maximum entropy, or equivalently, a minimum of the negative entropy. For isothermal systems, phase equilibrium occurs at the minimum of the Gibbs free energy. The entropy and Gibbs free energy of the phases are derived from the chemical potential $\boldsymbol{\mu}_{\alpha}$. These chemical potentials are typically specified in terms of an equation of state to model the dependence of pressure on temperature, composition, and specific volume of the phase. For a more detailed discussion see, for example, Michelsen and Mollerup (2004), Brantferger et al. (1991).

The chemical potentials $\boldsymbol{\mu}_{\alpha}$ are functions of $p_{\alpha}, T$, and phase composition $\boldsymbol{n}_{\boldsymbol{\alpha}}$. (Here, we have preserved the role of capillary pressure in determining the thermodynamic behavior of the system. One simplification is to define the thermodynamics in terms of the reference pressure and retain capillary pressure effects only in the definition of phase velocities, see Brantferger et al. (1991).) Furthermore the major thermodynamic variables describing each phase can all be expressed in terms of the phase's chemical potential. In particular, the partial molar entropy's are given by

$$
\boldsymbol{\sigma}_{\boldsymbol{\alpha}}=-\left(\frac{\partial \boldsymbol{\mu}_{\alpha}}{\partial T}\right)_{\boldsymbol{X}_{\boldsymbol{\alpha}, p_{\alpha}}}
$$

where $\boldsymbol{X}_{\boldsymbol{\alpha}}$ are the mole fractions and the partial molar enthalpies are given by

$$
\boldsymbol{h}_{\boldsymbol{\alpha}}=\left(\boldsymbol{\mu}_{\alpha}+T \boldsymbol{\sigma}_{\boldsymbol{\alpha}}\right) \text {. }
$$


Here the phase equilibrium problem is to determine the molar composition of the phases $\boldsymbol{n}_{\boldsymbol{\alpha}}$ given the total moles $\boldsymbol{n}$, pressure $p$ and the total enthalpy $H_{t}$. The equilibrium distribution of the components is given by minimizing the negative entropy of the system. In a general multiphase setting this becomes

$$
\min -\mathrm{S}=-\sum_{\alpha} \boldsymbol{n}_{\alpha}^{\mathrm{T}} \boldsymbol{\sigma}_{\alpha}
$$

subject to

$$
\boldsymbol{n}=\sum_{\alpha} \boldsymbol{n}_{\alpha}
$$

and

$$
H_{t}=(1-\phi) \rho_{r} h_{r}+\phi\left(\sum_{\alpha} \boldsymbol{n}_{\alpha}^{T} \boldsymbol{h}_{\alpha}\right)
$$

along with inequality constraints $\left(\boldsymbol{n}_{\boldsymbol{\alpha}} \geq 0\right)$ guaranteeing non-negativity of the compositions and thermal stability of the fluid $\left(\boldsymbol{n}_{\boldsymbol{\alpha}}{ }^{T} \partial \boldsymbol{h}_{\boldsymbol{\alpha}} / \partial T>0\right)$. (As noted above, if we do not consider the solid and the fluids to be in thermal equilibrium then we hold the total fluid enthalpy, $H_{f}=$ $\boldsymbol{n}_{l}^{T} \boldsymbol{h}_{l}+\boldsymbol{n}_{v}^{T} \boldsymbol{h}_{v}$, constant rather than the total enthalpy).

Treating this minimization problem, typically referred to as an isenthalpic flash calculation, has been discussed in the literature (see Brantferger (1991); Michelsen and Mollerup (2004); Michelsen (1999)) and will not be discussed in detail here. it is worth noting, however, that the Hessian of the negative entropy is a rank one perturbation of the Hessian of the Gibbs free energy, which indicates that the two functions are closely related.

In addition, at equilibrium the chemical potentials are equal; for example, in a two phase systems where the phases are denoted by $l$ and $v$,

$$
\boldsymbol{\mu}_{l}\left(\mathbf{X}_{l}, T, p_{l}\right)=\boldsymbol{\mu}_{v}\left(\mathbf{X}_{v}, T, p_{v}\right) .
$$

Here, the chemical potentials are typically specified in terms of mole fractions rather than moles; however, we can use the definition of mole fractions to express them in this form.

In addition to determining the composition of the phases and the temperature, the phase behavior also determines the properties of the phases. In particular, given pressure, temperature, and component molar densities, we can compute the volume occupied by the phases. To complete the mathematical formulation of the system we require that the sum of the phase volumes match the available pore volume according to the relation

$$
1=\sum_{\alpha} s_{\alpha}\left(p_{\alpha}, T, \boldsymbol{n}_{\alpha}\right)
$$

This equation constrains the evolution of the component conservation and energy equations. Here we have implicitly used the capillary pressure to relate the phase pressures to the reference pressure. Note that the $s_{\alpha}$ can be expressed in terms of the total moles per pore volume of the phase, given by

$$
n_{\alpha}^{T}=e^{T} \mathbf{n}_{\alpha}
$$

where $e$ is a vector of 1 's, divided by a molar density, $\rho_{\alpha}^{\prime}$; thus,

$$
s_{\alpha}=\frac{n_{\alpha}^{T}}{\rho_{\alpha}^{\prime}} \text {. }
$$




\subsection{Reactive Transport}

Chemical reactions may take place within a single fluid phase or between different fluid phases and between solids and fluids. Consider a mixture consisting of various fluid phases designated by $\alpha$ and solid grains with each solid phase designated by the index $m$. For convenience it is assumed that the same set of species are present in all fluid phases. Solid-Solid reactions are assumed to be mediated through one or more fluid phase. Reactions within a single fluid phase are referred to as homogeneous and all other reactions that involve fluid-fluid or fluid-solid reactions as heterogeneous. Homogeneous reactions can be written in canonical form in terms of a set of basis or primary species $\left\{\mathcal{A}_{j \alpha}\right\}$ as (Lichtner, 1985)

$$
\sum_{i} \nu_{j i} \mathcal{A}_{j \alpha} \rightleftharpoons \mathcal{A}_{i \alpha}
$$

with reaction rate $I_{i \alpha}$, stoichiometric reaction coefficients $\nu_{j i}$ and secondary species $\mathcal{A}_{i \alpha}$. Such reactions are often fast and their rates may be represented through equilibrium mass action relations (see Section 5.1).

Heterogeneous reactions take the form of reaction of a fluid phase with minerals which can be written in canonical form as

$$
\sum_{j} \nu_{j m} \mathcal{A}_{j \alpha} \rightleftharpoons \mathcal{M}_{m}
$$

for mineral $\mathcal{M}_{m}$ with reaction rate $I_{m \alpha}$ and stoichiometric coefficients $\nu_{j m}$. Here it is assumed that different fluid phases may react with the same minerals in the solid aggregate. These reactions are represented as a parallel reaction network with potentially different reaction mechanisms. Since mineral-water reactions tend to be slow, they are generally represented with kinetic rate laws (Steefel and Lasaga, 1994).

In addition, phase transformations between different fluid phases may take place described by reactions of the form

$$
\mathcal{A}_{j \alpha} \rightleftharpoons \mathcal{A}_{j \beta},
$$

between phases $\alpha$ and $\beta$. If $I_{j}^{\alpha \beta}$ denotes the rate of reaction (2.28), then it follows that the rate is antisymmetric in the phases $\alpha$ and $\beta$

$$
I_{j}^{\alpha \beta}=-I_{j}^{\beta \alpha}
$$

For a system of $N_{p}$ phases, there are a total of $2^{N_{p}}-1$ different equilibrium phase combinations. The total number of independent degrees of freedom is independent of the number of phases and equal to $N_{C}+1$, where $N_{C}$ denotes the number of independent chemical components. All reaction rates are assumed to have units of moles and are converted to mass units by multiplying by the appropriate formula weights $W_{j}, W_{i}$, and $W_{m}$ associated with the subscripted species.

To develop mass conservation equations for the reacting constituents, phase $\alpha_{\circ}$ is chosen as a reference phase with respect to which interphase mass transfer is described according the reaction given in Eqn. (2.28). In this treatment it is assumed that phase $\alpha_{\circ}$ refers to the aqueous phase. For a mass-based description and taking into account kinetic mass transfer rates for homogeneous and heterogeneous reactions, Eqns.(2.26) and (2.27), the mass balance equations for each primary 
species can be written as

$$
\begin{aligned}
\frac{\partial}{\partial t}\left(\phi s_{\alpha_{\circ}} \rho_{j \alpha_{\circ}}\right)+\nabla \cdot\left(\phi s_{\alpha_{\circ}} \rho_{j \alpha_{\circ}} \boldsymbol{v}_{j \alpha_{\circ}}\right)= & -W_{j} \sum_{i} \nu_{j i} I_{i \alpha_{\circ}} \\
& -W_{j} \sum_{\beta \neq \alpha_{\circ}} I_{j}^{\alpha_{\circ} \beta} \\
& -W_{j} \sum_{m} \nu_{j m} I_{m \alpha_{\circ}}, \\
\frac{\partial}{\partial t}\left(\phi s_{\beta} \rho_{j \beta}\right)+\nabla \cdot\left(\phi s_{\beta} \rho_{j \beta} \boldsymbol{v}_{j \beta}\right)= & -W_{j} \sum_{i} \nu_{j i} I_{i \beta} \\
& +W_{j} I_{j}^{\alpha_{\circ} \beta} \\
& -W_{j} \sum_{m} \nu_{j m} I_{m \beta},
\end{aligned}
$$

and for each secondary species as

$$
\frac{\partial}{\partial t}\left(\phi s_{\alpha} \rho_{i \alpha}\right)+\nabla \cdot\left(\phi s_{\alpha} \rho_{i \alpha} \boldsymbol{v}_{i \alpha}\right)=W_{i} I_{i \alpha}
$$

Changes in mineral concentrations are described by the equations

$$
\frac{\partial \phi_{m}}{\partial t}=\bar{V}_{m} \sum_{\alpha} I_{m \alpha}
$$

with molar volume $\bar{V}_{m}$ and where the sum over $\alpha$ on the right-hand side is over all fluid phases that react with the $m$ th mineral. It is assumed that the solid phase is stationary, an assumption which must be relaxed in order to incorporate mechanical deformation effects.

In these equations $\boldsymbol{v}_{i \alpha}$ denotes the mean velocity of the $i$ th species in phase $\alpha$. The velocity $\boldsymbol{v}_{\alpha}$ of each fluid phase $\alpha$ may be represented by the baryocentric form defined as

$$
\boldsymbol{v}_{\alpha}=\frac{1}{\rho_{\alpha}} \sum_{k} \rho_{k \alpha} \boldsymbol{v}_{k \alpha} .
$$

The diffusive flux $\boldsymbol{J}_{k \alpha}^{D}$ is defined as

$$
\begin{aligned}
\boldsymbol{J}_{k \alpha}^{D} & =\rho_{k \alpha}\left(\boldsymbol{v}_{k \alpha}-\boldsymbol{v}_{\alpha}\right) \\
& =-\rho_{\alpha} D_{\alpha} \boldsymbol{\nabla} X_{k \alpha}
\end{aligned}
$$

with the property

$$
\sum_{k} \boldsymbol{J}_{k \alpha}^{D}=0
$$

Note that for simplicity it is assumed that the diffusion coefficient $D_{\alpha}$ is species-independent and only depends on the particular phase $\alpha$. This assumption is generally not correct and the effects of a local electric field must be taken into account in a more general treatment involving diffusion 
of charged species to maintain local charge balance (Section 4.6). With this definition of diffusive flux one has

$$
\rho_{k \alpha} \boldsymbol{v}_{k \alpha}=\rho_{\alpha} X_{k \alpha} \boldsymbol{v}_{\alpha}+\boldsymbol{J}_{k \alpha}^{D} .
$$

It should be noted that the choice of the baryocentric velocity is arbitrary and other definitions are also possible. Introducing the Darcy velocity $\boldsymbol{q}_{\alpha}$ for each fluid phase defined as

$$
\boldsymbol{q}_{\alpha}=\phi s_{\alpha} \boldsymbol{v}_{\alpha}
$$

the flux becomes

$$
\rho_{k \alpha} \boldsymbol{v}_{k \alpha}=\frac{1}{\phi s_{\alpha}} \rho_{\alpha} X_{k \alpha} \boldsymbol{q}_{\alpha}+\boldsymbol{J}_{k \alpha}^{D}
$$

Summing Eqns.(2.30)-(2.32) over all species and fluid phases yields the overall mass conservation equation

$$
\frac{\partial}{\partial t}\left(\phi \sum_{\alpha} s_{\alpha} \rho_{\alpha}\right)+\nabla \cdot\left(\phi \sum_{\alpha} \rho_{\alpha} \boldsymbol{v}_{\alpha}\right)=0,
$$

where the reaction rate terms disappear through conservation of mass of each chemical reaction

$$
\sum_{j} W_{j} \nu_{j i}=W_{i}
$$

and

$$
\sum_{j} W_{j} \nu_{j m}=W_{m}
$$

Typically, the molar concentration defined in Eqn.(2.3) is used in place of mass concentration. In this case under the assumption of incompressible flow the diffusive flux can be written approximately as

$$
\boldsymbol{J}_{k \alpha}^{D} \simeq-D_{\alpha} \nabla C_{k \alpha}
$$

which is Fick's First Law.

\subsubsection{Partial Equilibrium}

Typically the reaction rates $I_{i \alpha}$ for secondary species are transport-controlled and may be determined by imposing conditions of local equilibrium through algebraic mass action constraints. In this case the rates $I_{i \alpha}$ can be eliminated by substituting Eqn.(2.32) into Eqns.(2.30) and (2.31). Summing over all fluid phases and assuming molar concentration variables are used this yields conservation equations for the primary species given by

$$
\sum_{\alpha}\left\{\frac{\partial}{\partial t}\left(\phi s_{\alpha} \Psi_{j \alpha}\right)+\nabla \cdot \Omega_{j \alpha}\right\}=-\sum_{\alpha m} \nu_{j m}^{\alpha} I_{m \alpha},
$$

which may be written alternatively as

$$
\frac{\partial}{\partial t} \phi \sum_{\alpha} s_{\alpha} \Psi_{j \alpha}+\nabla \cdot \sum_{\alpha} \Omega_{j \alpha}=-\sum_{\alpha m} \nu_{j m}^{\alpha} I_{m \alpha},
$$


where the total concentration $\Psi_{j \alpha}$ and flux $\Omega_{j \alpha}$ are defined, respectively, by

$$
\Psi_{j \alpha}=\delta_{\alpha_{\circ} \alpha} C_{j \alpha}+\sum_{i} \nu_{j i} C_{i \alpha}
$$

and

$$
\boldsymbol{\Omega}_{j \alpha}=\delta_{\alpha_{\circ} \alpha} \boldsymbol{J}_{j \alpha}+\sum_{i} \nu_{j i} \boldsymbol{J}_{i \alpha}
$$

where $\boldsymbol{J}_{j \alpha}$ and $\boldsymbol{J}_{i \alpha}$ denote the solute flux for primary and secondary species, respectively.

\subsection{Fractured and Highly Heterogeneous Media}

Important processes exist at the pore scale that can affect flow and transport at the continuum scale. For example, conceptual models of fracture flow and rock-water chemical interactions have been developed and studied at various scales. Although these models are considered a priority for later years, it is important to provide background information that may impact the current requirements and design documents. Thus, the following discussion presents a high-level overview of conceptual models used in fractured and highly heterogeneous media.

\subsubsection{Overview}

The fact that subsurface flow and transport occurs in an extremely heterogeneous material environment, which is often hierarchical in nature, has led to the multi-continuum class of models. Broadly speaking, these models separate the subsurface into several distinct materials based on flow and transport properties. For example fractures and clay layers have a permeability value that is orders of magnitude different than the surrounding rock or soil. Depending on the application, the properties of the different continua can be averaged into an "upscaled" property or left distinct. Other approaches have been taken to handle heterogeneities and transport occurring at small scales. For example, the field of stochastic hydrology attempts to quantify the impact of small-scale permeability heterogeneity by casting the governing equations for hydrologic flow and solute transport as a function of random variables (Zhang, 2001). When multiple continua remain distinct, sub-grid-scale processes arise. While this section is concerned with process models and not numerical grids, we can assume that at almost all grid resolutions, there is need, based on the discussion above, that several materials and processes will need to be represented in a single gridblock. For example, fracture widths are often on the order of millimeters while a fine grid in a basin-scale simulation may be on the order of meters. Sub-grid-scale models address this need. These models include common double permeability (DK), double porosity (DP, Barenblatt et al. (1960); Warren and Root (1963)), Multiple interacting continua (MINC, Pruess and Narasimhan (1985)), and Generalized Double Porosity Method (GDPM, Zyvoloski et al. (2008)).

Permeability in soils and rocks can be dominated by fine scale structure such as fractures, macropores, and clay layers. Fractures and macropores can be thought of conceptually as globally connected flow paths surrounded by material that acts as fluid storage. Similarly, clay layers can provide a contaminant release to a high permeability aquifer through rate-limited diffusion; the double porosity conceptualization has been validated by field and laboratory data and under isothermal 
conditions. The original development of dual porosity models began with the work of Barenblatt et al. (1960) who recognized that in fractured porous media, the fractures provide the primary conduit for mass transport, whereas the rock matrix could be represented as a fluid storage medium. In such a system the normal mass and energy balance equations can be written for the fracture domain, and an additional equation is written for the matrix node, which is connected only to its corresponding fracture node in a numerical model. In this formulation, the matrix is not a continuous medium in which the full mass and energy transport equations are solved. Nevertheless, important fluid and energy storage terms within the matrix blocks can be explicitly included. A significant limitation of the dual porosity (DP) concept is that although a matrix node is introduced to capture storage in the medium surrounding each fracture, there is no ability to capture gradients into the matrix with a single node. The solution to the original dual porosity models are sometimes called "quasi-steady" matrix solutions because of this limitation. Pruess and Narasimhan (1985) and Zyvoloski et al. (1997) extended the treatment of the matrix material by introducing a second matrix node.

Dual Permeability (DK) methods are like double porosity (DP) methods in that the grid-block is divided into distinct materials with different properties. Unlike DP methods, the secondary material is not only connected to the primary material, but also to other secondary material. This method is appropriate for non-isothermal and high-capillary pressure models. In these models heat conduction and capillary flow in the secondary (matrix) material is important. 


\section{Flow Processes}

\subsection{Overview}

In this section the basic flow models for single phase flow, Richards' equation and for multiphase flow are discussed, which is then followed by a section on infiltration. At the end of this section, data requirements for flow models are discussed, although this is a potentially very broad topic that can only be treated briefly here. Surface water flow and groundwater-surface water interactions will eventually be considered formally, since this is an important effect at many DOE sites, including Oak Ridge. At this stage, however, surface water is treated as a prescribed boundary condition.

\subsection{Assumptions and Applicability for Flow Equations}

Subsurface flow simulations typically assume that Darcy's law is valid. As this law gives a relationship between velocity and pressure, it essentially replaces the momentum equation. There has been much research to support the validity of Darcy's Law (?). Most references give the applicability of Darcy's Law to be for laminar flows with Reynolds numbers less that 10 using the pore throat diameter for a soil. There has been some effort to include inertial as well as turbulence effects that can occur near the wells.

It is also assumed that thermodynamic equilibrium (mechanical and thermal) exists for each grid block. Sub-grid scale features will often play a prominent role in multi-fluid simulations. Faults and fractures will likely be fast paths for contaminant transport and can effectively be treated with multiple porosity models. Similarly, rate-limited diffusion from clay inclusions can also be modeled with a multiple porosity material.

Modeling multiphase flow in liquid-gas systems is essential for many applications ranging from nuclear waste disposal involving boiling to problems involving a variably saturated zone with oxidation-reduction reactions taking place with consequent consumption of oxygen, for example. The EM complex includes a diversity of hydrologic settings that reflects the variability of geologic and climatic condition in the United States. The Hanford and Oak Ridge (or Savannah River) sites represent end members of these conditions. The Hanford site is very dry with a large vadose zone, a deep water table, and little atmospheric recharge. The Oak Ridge and Savannah River sites have wet conditions that result in a small vadose zone and a very shallow water table. The waste forms and contaminant sources vary from the simple (increased total dissolved solids) to the complex (multi-phase air-water-NAPL at Oak Ridge and Savannah River) to the daunting (mixed NAPL and radioactive waste at Rocky Flats and the Hanford site).

\subsection{General Formulation for Multiphase Flow}

The model equations that describe the flow of multiple fluid phases in the subsurface are a combination of the continuity equation and Darcy's law, which replaces the momentum equation. 


\subsubsection{Component Conservation Equations}

For each component, $j$, we have

$$
\frac{\partial}{\partial t}\left(\phi \sum_{\alpha} \rho_{\alpha} s_{\alpha} Y_{j \alpha}\right)+\nabla \cdot\left(\sum_{\alpha} \frac{\boldsymbol{k} k_{r \alpha} \rho_{\alpha} Y_{j \alpha}}{\mu_{\alpha}} \nabla\left(p_{\alpha}-\rho_{\alpha} \boldsymbol{g}\right)\right)=Q_{j},
$$

where $\mathbf{k}$ is the absolute permeability of the medium, $k_{r \alpha}$ is the relative permeability of phase, $\rho_{\alpha}$ and $s_{\alpha}$ are the density and saturation of phase $\alpha$ respectively, $Y_{j \alpha}$ is the mass fraction, and $Q_{j}$ is the net source term for component $j$.

\subsubsection{Relative Permeability and Capillary Pressure}

For both Richards' equation and more general multiphase problems, the model requires representations of relative permeability and capillary pressure. For two-phase systems, relative permeability and capillary pressure data are widely available for the more common forms such as van Genuchten-Mualem and Brooks-Corey.

In Equation (3.1) the individual phase pressures, $p_{\alpha}$, are related to a reference pressure $p$ by a capillary pressure $p_{c \alpha}$ that depends on saturation,

$$
p_{c \alpha}=p_{\alpha}-p .
$$

The capillary pressure is a function of saturation. Below we summarize two of the more common models for relative permeability and capillary pressure.

van Genuchten Capillary Pressure and Relative Permeability Relations. Effective liquid saturation described by the van Genuchten (1980) relation is given by

$$
s_{e}=\left[1+\left(\alpha\left|P_{c}\right|\right)^{n}\right]^{-m},
$$

where $P_{c}$ represents the capillary pressure $[\mathrm{Pa}]$, and the effective liquid saturation $s_{e}$ is defined further by

$$
s_{e}=\frac{s_{l}-s_{l}^{r}}{s_{l}^{0}-s_{l}^{r}}
$$

where $s_{l}$ denotes liquid saturation, $s_{l}^{r}$ denotes the residual saturation, and $s_{l}^{0}$ denotes the maximum saturation. The constants $n$ and $m$ are generally related by the expressions

$$
m=1-\frac{1}{n}, \quad n=\frac{1}{1-m} .
$$

The inverse relation is given by

$$
P_{c}=\frac{1}{\alpha}\left[\left(s_{e}\right)^{-1 / m}-1\right]^{1 / n} .
$$


The van Genuchten-Mualem relative permeability for the liquid phase is given by

$$
k_{r l}=\sqrt{s_{e}}\left\{1-\left[1-s_{e}^{1 / m}\right]^{m}\right\}^{2} .
$$

For the gas phase, the following formulation has been suggested

$$
k_{r g}=1-k_{r l},
$$

but a formulation proposed by Luckner et al. (1989) is often preferred and is given by

$$
k_{r g}=\left(1-s_{e k g}\right)^{1 / 3}\left[1-s_{e k g}^{1 / m}\right]^{2 m},
$$

where $s_{e k g}$ is defined in terms of the liquid saturation, $s_{l}$, and the residual gas saturation, $s_{g r}$, as

$$
s_{e k g}=\frac{s_{l}}{1-s_{g r}}
$$

Brooks-Corey Capillary Pressure and Relative Permeability Relations. Liquid saturation as described by the Brooks and Corey (1964) is given by

$$
s_{e}=\left(\alpha\left|p_{c}\right|\right)^{-\lambda}
$$

with the inverse relation given by

$$
p_{c}=\frac{1}{\alpha}\left(s_{e}\right)^{-1 / \lambda}
$$

Here $\lambda$ is a fitting parameter.

Brooks and Corey (1964) used the Burdine (1953) theory to derive an expression for the liquid relative permeability function given by

$$
\begin{aligned}
k_{r l} & =\left(s_{e}\right)^{(2+3 \lambda) / \lambda} \\
& =\left(\alpha\left|p_{c}\right|\right)^{-(2+3 \lambda)} .
\end{aligned}
$$

For the gas phase, the relative permeability is given by

$$
k_{r g}=\left(1-s_{e}\right)^{2}\left[1-s_{e}^{(2+\lambda) / \lambda}\right],
$$

Alternatively, both Equation (3.8) and Equation (3.9) have been used to describe the relative permeability of the gas phase.

Conservation Equations - Energy. For nonisothermal problems this system must be augmented with an energy equation. In the case of multi-phase flow, the conservation of energy equation takes a slightly different form from that given in Equation (2.13)

$$
\frac{\partial}{\partial t}\left[(1-\phi) \rho_{r} u_{r}+\phi \sum_{\alpha} \rho_{\alpha} u_{\alpha} S_{\alpha}\right]=\nabla \cdot \sum_{\alpha}\left(\frac{\boldsymbol{k} k_{r \alpha} \rho_{\alpha} h_{\alpha}}{\mu_{\alpha}}\right) \nabla\left(p_{\alpha}-\rho_{\alpha} \boldsymbol{g}\right)+\nabla \cdot Q_{T}+Q_{e}
$$

where: $\rho_{r}$ is the rock density, $u_{r}$ is the internal energy of the rock, $u_{\alpha}$ is the internal energy of phase $\alpha, h_{\alpha}$ is the enthalpy of phase $\alpha, Q_{T}$ represents thermal conduction and radiation, and $Q_{e}$ is a source or sink of energy. 
Closure and Constraint Equations. The equations above represent the conservation of water mass, the conservation of the $j$ th non-aqueous component, and the conservation of energy. These equations are augmented by the following constraints. The mass fractions (or equivalently, the mole fractions) in each phase must sum to 1 :

$$
\sum_{j} Y_{j \alpha}=1
$$

The phase saturations must sum to 1 :

$$
\sum_{\alpha} s_{\alpha}=1
$$

An equation of state is used to specify the phase behavior and to supply additional fluid properties.

\subsection{Richards' Equation}

\subsubsection{Overview}

Richard's equation is often used to describe single phase flow under partially saturated conditions (i.e., the pores are not occupied exclusively by a single phase). As such, it requires the introduction of a relative permeability and a capillary pressure relations as discussed in Section 3.3.2. Richards' equation is well suited to very large numerical problems (millions of degrees of freedom) because it requires only one independent variable per cell. This is accomplished by assuming a static gas phase. There are limitations to this approach. For example, when light or chlorinated hydrocarbons are present, even in very dilute quantities, the accurate representation of Henry's partitioning into the vapor (air) phase can be important. With Richards' equation, the partitioning can be represented, but the subsequent dilution due to air movement cannot. Another problem that can arise in deeper saline aquifers is the change in water density due to changes in brine concentration. In this case, another material balance equation can be introduced and solved in a fully coupled manner (with additional CPU and memory requirements) or the density variation can be obtained in a less coupled manner such as explicitly solving the flow and transport independently and calculating the density as a function of concentration. This method, while easier, has numerical stability concerns. As noted above, a variable density precludes the use of a head formulation for Richards' equation.

Assumptions and Applicability. Richards' equation makes the fundamental assumption that we are neglecting the movement of the gas phase. Because of this assumption, using Richards' equation may limit the kinds of transport analysis that can be done. It should also be noted that Richards' equation is often highly nonlinear with relative permeability of the liquid (most commonly water) phase, $k_{r l}$ (in turn a function of the liquid saturation, $s_{l}$ ) and the liquid pressure, $p_{l}\left(s_{w l}\right)$.

There are limitations to this approach. For example, when light or chlorinated hydrocarbons are present, even in very dilute quantities, the accurate representation of Henry's partitioning into the vapor (air) phase can be important. With Richards' equation, the partitioning can be represented, but the subsequent dilution due to air movement cannot. Another problem that can arise in deeper saline aquifers is the change in water density due to changes in brine concentration. In this case, 
another material balance equation can be introduced and solved in a fully coupled manner (with additional CPU and memory requirements) or the density variation can be obtained in a less coupled manner such as explicitly solving the flow and transport independently and calculating the density as a function of concentration. This method, while simpler, has numerical stability concerns. As noted above, a variable density precludes the use of a head formulation for Richards' equation. Finally, it is often the case that Richards' equation is presented in terms of hydraulic head instead of pressure.

\subsubsection{Process Model Equations}

Richards' equation provides the necessary physics to represent the flow of a liquid phase (typically water) under partially saturated conditions, with the assumption made that the second phase is inactive. Richards' equation is derived from the conservation of liquid mass continuity equation:

$$
\frac{\partial\left(\phi s_{l} \rho_{l}\right)}{\partial t}=\nabla \cdot\left(\rho_{l} \boldsymbol{q}_{l}\right)+Q_{l}
$$

where $\rho_{l}$ is the liquid density, $Q_{l}$ is a source or sink, and $\boldsymbol{q}_{l}$ is the liquid velocity given by Darcy's Law. This formulation differs from Equation (3.22) only in adding a relative permeability of the liquid, $k_{r l}$

$$
\boldsymbol{q}_{l}=-\frac{\boldsymbol{k} k_{r l}}{\mu_{l}}\left(\nabla p_{l}-\rho_{l} \boldsymbol{g}\right)
$$

where $\boldsymbol{k}$ is the intrinsic rock or soil permeability, $\mu_{l}$ is the liquid viscosity, $p_{l}$ is the liquid pressure (or capillary pressure), and $\boldsymbol{g}$ is the acceleration of gravity.

These equations are usually combined to form the traditional form of Richards' equation:

$$
\frac{\partial\left(\phi s_{l} \rho_{l}\right)}{\partial t}=\boldsymbol{\nabla} \cdot\left[\frac{K k_{r l} \rho_{l}}{\mu_{l}}\left(\boldsymbol{\nabla} p_{l}-\rho_{l} \boldsymbol{g}\right)\right]+Q_{l} .
$$

Typical models of relative permeability as a function of saturation are the van Genuchten-Mualem relations (Equation (3.7)) and the Brooks-Corey relations (Equation (3.13)).

\subsection{Single-Phase Flow}

\subsubsection{Overview}

The most basic case of flow is that of a single phase in a porous medium. Notwithstanding its simplicity, it has a wide application to describing subsurface processes. As we described in Section 2, the mathematical treatment is based on Darcy's Law for momentum balance for flow in a porous medium.

Assumptions and Applicability. There are many assumptions required for the strict validity of Darcy's Law, including incompressible, laminar flow. 


\subsubsection{Process Model Equations}

For single phase flow in porous media under isothermal conditions the general formulation discussed in section 2 reduces to

$$
\frac{\partial}{\partial t}\left(\phi \rho_{l}\right)+\nabla \cdot\left(\rho_{l} \boldsymbol{q}\right)=Q
$$

where $\rho_{l}$ denotes the fluid density (sometimes considered to be a function of $p$ ), $Q$ represents a source/sink term and $\boldsymbol{q}$ is the Darcy velocity

$$
\boldsymbol{q}=-\frac{k}{\mu_{l}}(\boldsymbol{\nabla} p-\rho \boldsymbol{g}),
$$

In place of this formulation for Darcy's Law, it is common to see it written in terms of hydraulic head defined as:

$$
h=z+\frac{p}{\rho_{l} g} .
$$

Use of the hydraulic head as the key variable leads naturally to the use of the hydraulic conductivity, $\mathrm{K}$, defined as

$$
K=\frac{k \rho_{l} g}{\mu_{l}} .
$$

In the case where the density and viscosity are constant, therefore, it is possible to write Darcy's Law as

$$
\boldsymbol{q}=-K \nabla h .
$$

\subsection{Infiltration}

\subsubsection{Overview}

The infiltration process models are components of the subsurface fluid migration. Infiltration can be an important driving force for contaminant transport, especially in the vadose zone. Engineered subsurface barrier technology seeks to minimize the infiltration driving force for contaminant migration. There are a number of approaches and models that can be applied to predict infiltration processes. These range from simple storage routing models to the more mechanistic Richards' equation-based models that simulate water flow and heat transport in response to meteorological forcing and plant water uptake. Within the complex interaction of physical, hydrologic, and biotic processes that control field-scale infiltration at the site of interest, the ideal model should be capable of assessing the impact of infiltration on contaminant transport, as well as supporting barrier design and performance assessment.

Predicting infiltration requires consideration of unsaturated flow processes, precipitation, snow accumulation and melting, surface runoff, water storage, evaporation, transpiration, lateral diversion along sloped layers, and, ultimately, deep percolation (Ward and Gee, 1997; Ward and Keller, 2005). All of these processes occur in response to forcing meteorology that leads to temporal variability in air temperature, relative humidity, wind speed, and barometric pressure and, in the most sophisticated implementations, require the solution of coupled equations for mass and energy transport. 
A minimum set of processes for modeling the water budget should include:

$$
P+I=R_{\text {over }}+\Delta W+D+G D+E+T,
$$

where:

$P=$ precipitation

$I=$ irrigation

$R_{\text {over }}=$ overland flow (run-off and run-on)

$D=$ drainage out of the soil cover (diverted by reduced-permeability layer)

$G D=$ ground water recharge (deep percolation past a reduced-permeability layer)

$\Delta W=$ change in soil water storage

$E=$ evaporation

$T=$ transpiration .

Evaporation is defined as the process by which liquid water is transformed into a gaseous state and the subsequent transfer of this vapor to the atmosphere. Transpiration is the loss of water from plants through their stomata to the atmosphere. Plants compensate for transpiration losses by taking up water from the soil.

Another requirement is that the process models must include a full energy balance (nonisothermal) option for evapotranspiration processes. Water that does not run off the surface must be available for evaporation from the soil or plant surfaces, or infiltration into the soil profile. Soil water content must depend on the interactions of precipitation, temperature, vegetation, and albedo changes that vary temporally (e.g., diurnally, seasonally, and episodically). Spatially and temporally variable water storage and flux must be available for contaminant transport.

\subsubsection{Process Model Requirements}

Precipitation. The treatment of precipitation must include all natural sources of moisture that may reach the surface in the form of rain, snow, sleet, hail, dew, and fog, and must account for precipitation not available for infiltration. This includes precipitation intercepted by the plant canopy, from which it is evaporated or transpired without ever contacting the soil; and sublimation, the direct conversion of water from the solid phase to the vapor phase. This should also account for the presence of a snow cover that can delay infiltration, reduce evaporation rates, and in the event of rapid snowmelt, lead to surface runoff.

Non-Precipitation Surface Recharge (including leaks). Process models should account for surface recharge sources (e.g., irrigation water used during construction as a dust control agent and post-construction to support the establishment of vegetation; water condensing on plant surfaces and falling to the ground once the maximum storage depth in the canopy is exceeded, pipe leaks). These sources must also be subject to evaporation from soil and plant surfaces with the remainder becoming available for runoff or infiltration. 
Evapotranspiration. Evapotranspiration models should include all the processes that convert water from the aqueous phase into water in the gaseous phase, i.e., water vapor. This should also account for evaporation from soil and plant surfaces and plant transpiration and include options where these components vary with soil properties and structure of the plant canopy.

Evaporation. In particular, evaporation models should account for surface wetness controls on evaporation for soil and plant surfaces. This should be done using the concept of potential evaporation, the amount of water that could be evaporated were it freely available under the energy and atmospheric conditions (i.e., function of surface and air temperatures, insolation, and wind speed) that control water-vapor concentrations immediately above the evaporating surface. Actual evaporation will be calculated as a fraction of potential evaporation that accounts for the water availability and humidity differences between the atmosphere and the evaporating surface. Evaporation from wet vegetated surfaces will depend on the amount of water that has accumulated on the leaves and stems following precipitation events. The evaporation rate will be determined by the amount of energy available, i.e., the solar radiation that is intercepted, the relative humidity of the air, and the vapor pressure of the air above the evaporating surface. For evaporation to occur, the vapor pressure of the free water in or on plants must be greater than the vapor pressure of water in the air. Finally, models should be able to account for air flow over the plant surfaces.

Transpiration. Transpiration models need to account for passive transpiration processes controlled by the humidity of the atmospheric and the moisture content of the soil. To take advantage of existing databases, use the concept of potential and actual transpiration. The potential transpiration rate depends on the leaf area and the evaporative demand of the atmosphere, assuming soil water is not limiting. The evaporative demand will be a function of incoming solar radiation and its partitioning into sensible and latent heat fluxes, vapor pressure deficit, and wind speed. Actual transpiration is the water limited transpiration rate, and the ratio between actual and potential transpiration is indicative of the extent to which the plant suffers from water stress. Actual transpiration will be a function of temperature and root water uptake reductions caused by water stress.

\subsubsection{Plant Interactions}

Root Water Uptake. Account for water uptake by plants from the soil to compensate for transpiration losses. Include stomatal opening and closing to control water loss and the capability to take up water held at very high matric potentials.

Canopy Interception. Models should account for precipitation falling on vegetative surfaces, i.e., the plant canopy, that collects on these surfaces. They should also be able to account for intercepted water being absorbed by plant surfaces, evaporated from these surfaces, or eventually dripped to the ground surface after the interception capacity is exceeded. Include options for specifying relationships between interception of rainfall and rainfall intensity. 


\subsection{Data Requirements for Flow}

\subsubsection{Permeability and Porosity}

The most basic properties of the subsurface, which are required for all of the flow models, are permeability and porosity. Often this data can only be provided by sparsely located well logs and at a spatial scale much finer than the scale of the model. As a consequence, techniques of upscaling are required to fill in the missing data and extrapolate to larger scales.

Permeability estimates are available for a large variety of soils and rocks. When conceptual (and numerical) models have cells that represent fractures or faults, field tests (pumping and/or tracer) are required to determine effective permeabilities. Porosity data is also available for many rock and soil types.

\subsubsection{Relative Permeability and Capillary Pressure.}

For both Richards' equation and more general multiphase problems, the model requires representations of relative permeability and capillary pressure. For two-phase systems, relative permeability and capillary pressure data are widely available for the more common forms such as van Genuchten and Brooks Corey.

When a NAPL phase is present and the likelihood of three phases is significant, much of the relative permeability data available from the soil literature may have only limited applicability and experiments on at least core size sample will be necessary. Stone (1973) presented a method to estimate three-phase relative permeability that is in common usage in the oil industry. However, when fractures or faults are present, parameters of the relative permeability and capillary pressure models are estimated with field data. Relative permeabilities often exhibit strong hysteretic behavior. Land's method (Land, 1968; Spiteri and Juanes, 2006) is one approach for handling hysteretic behavior that is relatively simple to implement and is commonly used in the oil industry.

Some capillary pressure data is available in the database described by Schaap et al. (2001). Capillary models derived using surface tension data of pure components (Prausnitz et al., 1977) are used where experimental data is not available.

\subsubsection{Fluid Characterization}

The model equations must also be augmented with property data for the fluids. In simple problems we require only estimates of density and viscosity for each of the flowing phases. For more complex problems in which we are modeling a multicomponent system, additional data is required.

Equation of State. For multicomponent system, equation of state (EOS) data is required for water and all the NAPL components. Typically these are EOS for pure substances that are combined for mixtures. A basic EOS relates density to pressure and temperature. The form for the EOS is typically cubic such as the Soave-Redlich-Kwong (SRK) or the Peng-Robinson (PR) models. Tabular models are also in common use. 
Mixture thermodynamics is used to combine pure phase EOS's for the application. The EPA lists over 80 potential NAPL components (volatile organic compounds or VOCs) that can cause groundwater contamination and has data bases with at least some properties for these contaminants.

Mixture Internal Energy and Enthalpy. These will generally follow the simple additive rule based of component values and mass fraction. Consideration must be also given to heats of solution.

\subsubsection{Boundary Conditions and Initial Conditions}

Boundary and initial conditions can take several different forms depending on the application. Dirichlet boundary conditions specify the pressure, temperature, and saturation at the boundary. Neumann conditions specify the flux $\boldsymbol{q}$ at the boundary. Initial conditions may consist of specifying a constant pressure or variable pressure, temperature, and saturation, for example in the form of hydrostatic conditions taking into account the change in fluid density.

Typical boundary condition for Richard's equation consist of infiltration (recharge) and constant or time varying pressure conditions. More sophisticated conditions such at unit gradient, free drainage, and seepage face are also used.

\subsection{Reaction-Induced Porosity and Permeability Change}

\subsubsection{Porosity Changes}

Porosity changes in matrix and fractures are directly tied to the volume changes as a result of mineral precipitation and dissolution. The molar volumes of minerals created by hydrolysis reactions (i.e., anhydrous phases, such as feldspars, reacting with aqueous fluids to form hydrous minerals such as zeolites or clays) are often larger than those of the primary reactant minerals; therefore, constant molar dissolution-precipitation reactions may lead to porosity reductions.

The porosity, $\phi$, of the medium (fracture or matrix) can be calculated from

$$
\phi=1-\sum_{m=1}^{N_{m}} \phi_{m},
$$

where $N_{m}$ is the number of minerals, and $\phi_{m}$ is the volume fraction of mineral $m$ in the rock $\left(\mathrm{m}^{3}\right.$ mineral $\mathrm{m}^{-3}$ medium). As the volume fraction of each mineral changes due to mineral reactions, the porosity can be recalculated at each time step.

\subsubsection{Fracture Permeability Changes}

Fracture permeability changes can be approximated using the porosity change and an assumption of plane parallel fractures of uniform aperture (cubic law; Steefel and Lasaga (1994)). The 
modified permeability, $k$, is then given by

$$
k=k_{i}\left(\frac{\phi}{\phi_{i}}\right)^{3},
$$

where $k_{i}$ and $\phi_{i}$ are the initial permeability and porosity, respectively. This law yields zero permeability only under the condition of zero fracture porosity.

In most experimental and natural systems, permeability reductions to values near zero occur at porosities significantly greater than zero. This generally is the result of mineral precipitation in the narrower interconnecting apertures. The hydraulic aperture, as calculated from the fracture spacing and permeability (as determined through air-permeability measurements) assuming a cubic law relation, is a closer measure of the smaller apertures in the flow system. Using the hydraulic aperture, a much stronger relationship between permeability and porosity can be developed. This relationship can be approximated as follows:

The initial hydraulic aperture $\delta_{0, h}(m)$ is calculated using the following cubic law relation:

$$
\delta_{0, h}=\left[12 k_{0} s\right]^{\frac{1}{3}},
$$

where $k_{0}$ is the initial fracture permeability $\left(\mathrm{m}^{2}\right)$ and $s$ is the fracture spacing $(m)$. The permeability $\left(k^{\prime}\right)$ resulting from a change in the hydraulic aperture, is given by

$$
k^{\prime}=\frac{\left(\delta_{0, h}+\Delta \delta\right)^{3}}{12 s},
$$

where $\Delta \delta$ is the aperture change resulting from mineral precipitation/dissolution.

The aperture change resulting from a calculated volume change can be approximated by assuming precipitation of a uniform layer over the entire geometric surface area of the fracture, assuming also that this area as well as the fracture spacing remains constant. The actual distribution of mineral alteration is much more heterogeneous and depends on many processes that are active at scales much smaller than the resolution of the model; however, the combined effect of the initial heterogeneities and localized precipitation processes can only be treated through model sensitivity studies and experiments.

For a dual permeability model, changes in the fracture porosity are calculated based on the porosity of the fracture medium, so that $\Delta \delta$ can be approximated by

$$
\Delta \delta=\frac{\left(\phi_{f m}^{\prime}-\phi_{f m, 0}\right)}{\phi_{f m, 0}} \delta_{g} .
$$

\subsubsection{Matrix Permeability Changes}

Matrix permeability changes are calculated from changes in porosity using ratios of permeabilities calculated from the Carman-Kozeny relation (Bear, 1972a), and ignoring changes in grain size, tortuosity, and specific surface area as follows:

$$
k=k_{i} \frac{\left(1-\phi_{i}\right)^{2}}{(1-\phi)^{2}}\left(\frac{\phi}{\phi_{i}}\right)^{3} .
$$


The simple cubic law (Equation (3.28)) and the Kozeny-Carman (Equation (3.32)) porosity-permeability equations may not reflect the complex relationship of porosity and permeability in geologic media that depends on an interplay of many factors, such as pore size distribution, pore shapes, and connectivity (Verma and Pruess, 1988). Laboratory experiments have shown that modest reductions in porosity from mineral precipitation can cause large reductions in permeability (Vaughan, 1989). Detailed analysis of a large set of field data also indicated a very strong dependence of permeability on small porosity changes (Pape et al., 1999). This is explained by the convergent-divergent nature of natural pore channels, where pore throats can become clogged by precipitation while disconnected void spaces remain in the pore bodies (Verma and Pruess, 1988). The permeability reduction effects depend not only on the overall reduction of porosity, but on the details of the pore space geometry and the distribution of precipitates within the pore space. These may be quite different for different media, which makes it difficult to achieve generally applicable predictions. To evaluate the effects of a more sensitive coupling of permeability to porosity, we also implemented an improved porosity-permeability relationship presented by (Verma and Pruess, 1988).

$$
\frac{k}{k_{i}}=\left(\frac{\phi-\phi_{c}}{\phi_{i}-\phi_{c}}\right)^{n},
$$

where $\phi_{c}$ is the value of "critical" porosity at which permeability goes to zero, and $n$ is a power law exponent. Parameters $\phi_{c}$ and $n$ are medium-dependent.

\subsubsection{Effects of Permeability and Porosity Changes on Capillary Pressures}

Permeability and porosity changes will likely result in modifications to the unsaturated flow properties of the rock. Changes to unsaturated flow properties are approximated by modification of the calculated capillary pressure $\left(p_{c}\right)$ using the Leverett scaling relation (Slider, 1976) to obtain a scaled $p_{c}^{\prime}$ as follows:

$$
p_{c}^{\prime}=p_{c} \sqrt{\frac{k_{i} \phi}{k \phi_{i}}} .
$$




\section{Transport Processes}

\subsection{Overview}

Transport is arguably the single most important process that needs to be accurately captured in the Environmental Management modeling tool set. This is of course because the rate of transport determines the rate at which contaminants migrate to the biosphere. In what follows, we use "Transport" to refer to the set of physical processes that lead to movement of dissolved and solid contaminants in the subsurface, treating the chemical and microbiological reactions that can affect the transport rate through a retardation effect as a separate set of processes. The principal transport processes to be considered are advection, dispersion, and molecular diffusion. In addition, we consider electrochemical migration (Newman and Thomas-Alyea, 2004) as related to molecular diffusion, although it could be potentially treated as a separate flux at the same level as the others.

\subsection{Process Model Equations for Transport}

Recall the equation for mass conservation can be written as

$$
\frac{\partial\left(\phi \sum_{\alpha}\left[s_{\alpha} \boldsymbol{C}_{\boldsymbol{\alpha}}\right]\right)}{\partial t}+\boldsymbol{\nabla} \cdot \boldsymbol{J}_{\mathrm{adv}}=\boldsymbol{\nabla} \cdot \boldsymbol{J}_{\mathrm{disp}}+\boldsymbol{\nabla} \cdot \boldsymbol{J}_{\mathrm{diff}}+\sum_{i} Q_{i}
$$

where $\boldsymbol{J}_{\text {adv }}$ refers to the advective flux, $\boldsymbol{J}_{\text {disp }}$ is the dispersive flux, $\boldsymbol{J}_{\text {diff }}$ is the diffusive flux (often grouped with the dispersive flux), and $\sum_{i} Q_{i}$ is the summation of the various source terms (which may include reactions).

Assumptions and Applicability. The principal assumptions associated with the transport process models derive from the continuum treatment of the porous medium. Pore scale processes, including the resolution of variations in transport rates within individual pores or pore networks (Kang et al., 2006; Li et al., 2008), are generally not resolved, although some capabilities for treating multi-scale effects will be included in the HPC code. In general, it is assumed that within any one Representative Elementary Volume (REV) corresponding to a grid cell all transport rates are the same. It will be possible, however, to define overlapping continua with distinct transport rates, as in the case where the fracture network and rock matrix are represented as separate continua.

Coupling Considerations. Transport processes may be tightly coupled to both flow and reaction processes. In the case of flow, one important coupling is associated with the transport of chemical constituents that affect the density of the solution, which in turn affects flow rates through buoyancy.

In the case of chemical reactions, the coupling effect is normally very strong for reactive constitutents. Chemical reactions may consume components present in the gaseous phase (e.g., $\mathrm{CO}_{2}$ or $\mathrm{O}_{2}$ ), thus modifying the size of the phase itself. Or reactions can strongly modify gradients, and thus transport rates, by either consuming or producing various chemical species. 
Multiscale Considerations. The multiscale nature of porous media and the transport processes associated is arguably the most significant and largely unresolved challenge for simulation of fate and transport in subsurface aquifers. Transport actually operates at the pore scale where variations in flow velocity and reaction rates can result in microscopic variability in transport rates. Continuum treatments of transport in porous media cannot resolve such sub-grid variations easily, although various upscaling techniques may be available for capturing the smaller scale behavior. In addition, multi-continuum or hybrid approaches may obviate the need for a formal upscaling procedure, although there are significant computational difficulties and expense associated with their implementation.

\subsection{Boundary Conditions, Sources and Sinks}

A number of boundary conditions are possible for the diffusive flux, including a Dirichlet (or first-type) boundary condition (fixed concentration), a Neuman (or second-type or flux) boundary condition, and a Cauchy (or third-type) boundary condition.

First-Type or Dirichlet Boundary Condition: A first-type or Dirichlet condition involves specification of a fixed value (typically) of the concentration, $C_{0}$, at the boundary location,

$$
y(0)=C_{0}
$$

Second-Type or Neuman Boundary Condition: A second-type or Neuman (or flux) boundary condition involves specification of the derivative

$$
\frac{\partial C}{\partial x}(0)=\alpha
$$

where $\alpha$ is a prescribed value of the concentration gradient.

Third-Type or Robin Boundary Condition: A third-type or Robin boundary condition involves a specification of the value of the function itself and its derivative, so

$$
\alpha C+\beta \frac{\partial C}{\partial n}=\gamma
$$

where $\alpha$ and $\beta$ are prescribed values of the function and its derivative, respectively.

\subsection{Advective Transport}

\subsubsection{Overview}

The advection process models are components of the subsurface transport capability. Advection is the process where the bulk fluid motion transports mass and heat. In the simplest conceptualization of advection, the mass of a component in a fluid parcel simply moves with the velocity of the fluid parcel. This assumes there are no other processes (e.g., diffusion, dispersion, reactions) that can affect the component concentration in the fluid parcel. There are, however, situations where the advection is modified by attributes of the transported mass and the pore structure. One is the potential 
for nonreactive anions to be repulsed by negatively charged solid surfaces into the center of pore throats where the velocity is faster. Another is the advection of inorganic and organic colloids, and microorganisms, whose movement can be affected by the geometry of pore throats. In addition to being subject to the same physicochemical phenomena as abiotic colloids, microorganisms have biological processes that can affect advection (e.g., temporal changes in surface properties due to changes in metabolic state; chemotaxis; predation). We limit the discussion here to advection and exclude the attenuation mechanisms and microbial behaviors that are discussed elsewhere. Thus, advection can be a particulate or a dissolved species moving with the pore-water whose velocity is governed by the flow processes (discussed elsewhere). Continuum models have addressed these behaviors using bulk parameterizations to characterize the pore-scale controls and controlling chemical gradients. Sufficiently high concentrations of these advected components can affect the fluid properties which feedback to the flow processes.

The advection process can be modeled in isolation or as part of a transport process that includes diffusion, dispersion, and transformation processes, the latter typically represented as sources and sinks. Mathematically, advection is addressed in the divergence of mass fluxes across the surface of a control volume, $\boldsymbol{\nabla} \cdot(u c)$, where the fluxes are the product of concentrations of a given component and the fluid velocity at the control volume surface.

Numerical difficulties with the accuracy, robustness, and computation efficiency of modeling the advection of moving steep concentration fronts, especially in complex velocity fields, are well known. In some cases, there are constraints on the Peclet and Courant numbers for the useful application of a given technique. Without going into those difficulties and techniques, we specify the following requirements for the treatment of these issues.

\subsubsection{Process Model Equations}

Advection involves the translation in space of dissolved or suspended material at the rate of movement of the bulk fluid phase. No modification of the shape of a front and no dilution occurs when transport is purely via advection - a sharp front remains so when undergoing purely advective transport. The advective flux, $\boldsymbol{J}_{a d v}$, of a dissolved species in porous media can be described mathematically as

$$
\boldsymbol{J}_{a d v}=\phi s_{\alpha} \boldsymbol{v}_{\alpha} C_{i}
$$

where $\phi$ is the porosity, $s_{\alpha}$ is the saturation of phase $\alpha$, and $\boldsymbol{v}_{\alpha}$ is the average linear velocity of the phase, and $C_{i}$ is the concentration of the $i$ th species.

\subsection{Dispersive Transport}

\subsubsection{Overview}

Dispersion of a dissolved constituent refers to its spreading along tortuous pathways in a porous medium caused by mixing effects. Dispersion takes place in the direction of the flow (longitudinal) and normal to the flow (transverse). A conventional Eulerian Fickian representation of dispersion 
is assumed, which may be taken as the asymptotic limiting form of the dispersion tensor (Neuman, 1990). It should be noted that issues related to the scale dependence of dispersion are not considered. While this approach is known to have several limitations, such as backward dispersion against the direction of flow and scale independence, nevertheless it is still widely used in practical applications. Furthermore, it may be the only approach for representing local scale dispersion.

\subsubsection{Process Model Equations}

The dispersion tensor takes different forms depending on whether the media is isotropic or anisotropic.

Isotropic Media. For an isotropic medium there is no preferred axis of symmetry the dispersion tensor $\boldsymbol{D}^{H}$ has the well-known form (Bear, 1972b)

$$
\boldsymbol{D}^{H}=\alpha_{T} v \boldsymbol{I}+\left(\alpha_{L}-\alpha_{T}\right) \frac{\boldsymbol{v} \boldsymbol{v}}{v},
$$

where $\alpha_{L}[\mathrm{~m}]$ and $\alpha_{T}[\mathrm{~m}]$ refer to the longitudinal and transverse dispersivity, respectively, $\boldsymbol{v}[\mathrm{m} / \mathrm{s}]$ denotes the average pore velocity with magnitude $v$, and $\boldsymbol{I}$ is the identity matrix.

Anisotropic Media. The dispersion tensor for anisotropic media has not received much attention, However, it has been shown that in an axi-symmetric medium with axis of symmetry $\boldsymbol{\lambda}_{s}$, the dispersion tensor takes the general form (Lichtner et al., 2002)

$$
\begin{aligned}
\boldsymbol{D}^{H}=\alpha_{T}^{H} v \boldsymbol{I} & +\left[\alpha_{L}^{H}-\alpha_{T}^{V}+\cos ^{2} \theta\left(\alpha_{L}^{V}-\alpha_{L}^{H}+\alpha_{T}^{V}-\alpha_{T}^{H}\right)\right] \frac{\boldsymbol{v} \boldsymbol{v}}{v} \\
& +\left(\alpha_{T}^{V}-\alpha_{T}^{H}\right) v\left[\boldsymbol{\lambda}_{s} \boldsymbol{\lambda}_{s}-\frac{\cos \theta}{v}\left(\boldsymbol{\lambda}_{s} \boldsymbol{v}+\boldsymbol{v} \boldsymbol{\lambda}_{s}\right)\right]
\end{aligned}
$$

where $\alpha_{L}^{H, V}$ and $\alpha_{T}^{H, V}$ refer to the longitudinal and transverse dispersivity in the horizontal and vertical directions, and $\theta$ denotes the angle between the axis of symmetry and the flow velocity.

\subsection{Diffusive Transport}

\subsubsection{Overview}

Molecular diffusion is often indistinguishable from mechanical dispersion as a process operating in porous media, and thus the two are often lumped together to form a hydrodynamic dispersion term. Unlike dispersion, however, there is no effect of flow direction, so the potential difficulties associated with mismatches between flow and grid coordinate direction do not arise. Molecular diffusion is an entropy-producing process in which the random motion of molecules causes spreading or homogenization of a concentration field. Atomistic representations of molecular diffusion capture this random motion, but continuum models of the kind considered here typically represent only the average behavior of the molecules. It is noteworthy, however, that atomistic and continuum models for molecular diffusion do agree if sufficiently long time scales with a sufficient number of molecules are considered (Bourg et al., 2008). 
The principal distinctions in the treatment of molecular diffusion are between those based on Fick's Law, which states that the diffusive flux is linearly proportional to the concentration gradient, and multicomponent treatments that take into account the buildup of electrostatic forces as the individual charged species (ions) attempt to diffuse at their own rate. In addition, a full treatment of molecular diffusion involves calculating fluxes in terms of gradients in chemical potential rather than concentration (Steefel and Maher, 2009).

In addition to the complexities associated with chemical interactions, it is also necessary to account for the effects of the porous medium through which diffusion occurs. Corrections to the diffusive flux are often represented with a tortuosity (see Section 4.6.2 below) based on an upscaled constitutive law intended to capture the heterogeneous pore geometries. Since diffusion may be restricted or eliminated through narrow pore throats, the effective diffusivity for a specific ion may be quite different from its diffusivity in water alone. Capturing the multiscale nature of the pore structure and its effect on molecular diffusion remains a challenge.

\subsubsection{Process Model Equations}

General Formulation for Molecular Diffusion. The most rigorous and general expression for molecular diffusion is given by

$$
\boldsymbol{J}_{j}^{\text {diff }}=-\nabla\left(L_{j i} \mu_{j}\right),
$$

where the $L_{j i}$ are the phenomenological coefficients introduced in the theory of irreversible thermodynamics (Lasaga, 1998; Onsager, 1931; Prigogine, 1968) and $\mu_{j}$ is the chemical potential of the $j$ th species. Here, the fluxes are linearly related to gradients in the chemical potentials of the solutes rather than to their concentrations as in Fick's Law that follows. The phenomenological coefficients, $L_{j i}$, can be linked back to measurable quantities by making use of the mobility as the "velocity" of a particle acted upon by a force, with the force in this case provided by the chemical potential rather than the concentration

$$
\boldsymbol{J}_{j}^{\text {diff }}=-\nabla\left(u_{j} C_{j} \mu_{j}\right)
$$

where $u_{j}$ is the mobility of the $j$ th ion defined by

$$
u_{j}=\frac{D_{i}}{R T}
$$

Single Species Diffusion (Fick's Law). Molecular diffusion is usually described in terms of Fick's First Law, which states that the diffusive flux is proportional to the concentration gradient

$$
\boldsymbol{J}_{i}=-\nabla\left(D_{i} C_{i}\right)
$$

$D_{i}$ is referred to as the diffusion coefficient and is specific to the chemical component considered as indicated by the subscript $i$. Fick's First Law is a phenomenological theory for diffusion that relates diffusion to the "driving force" provided by the concentration gradient, although it can also be derived atomistically (Lasaga, 1998). In the case of diffusion in porous media, it is normally necessary to include a tortuosity correction as well (see discussion below). 


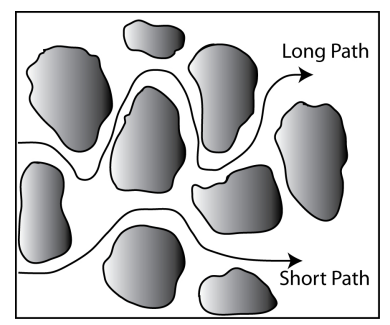

Figure 1: Tortuous diffusion paths in porous media (Steefel and Maher, 2009)

Tortuosity. Since water-rock interaction commonly takes place in porous materials, it is important to account for the effect of tortuosity (Figure 1), which is defined as the ratio of the path length the solute would follow in water alone, $L$, relative to the tortuous path length it would follow in porous media, $L_{e}$ (Bear, 1972b)

$$
T_{L}=\left(L / L_{e}\right)^{2}
$$

In this definition of tortuosity (sometimes the inverse of Equation (4.12) is used), its value is always $<1$ and the effective diffusion coefficient in porous media is obtained by multiplying the tortuosity by the diffusion coefficient for the solute in pure water.

With this formulation, the diffusion coefficient in porous media, $D_{i}^{*}$, is given by

$$
D_{i}^{*}=T_{L} D_{i}
$$

The diffusive flux, then, is given by

$$
\boldsymbol{J}_{j}^{\text {diff }}=-\nabla\left(\phi s_{\alpha} D_{j} T_{L} C_{j}\right)=-\nabla\left(\phi s_{\alpha} D_{j}^{*} C_{j}\right) .
$$

An alternative formulation for the coefficient for molecular diffusion in porous media is given by the formation factor, $F_{f}$, defined as (Bear, 1972b)

$$
F_{f}=\frac{1}{\phi s_{\alpha}}\left(\frac{L_{e}}{L}\right)^{2}=\frac{1}{\phi s_{\alpha}} T_{L},
$$

in which case the diffusive flux in porous media becomes

$$
\boldsymbol{J}_{j}^{\text {diff }}=-\nabla\left(\frac{D_{j}}{F_{f}} C_{j}\right)
$$

Various approaches for calculating formation factors (and thus, the diffusion coefficient in porous medium) are in use, with a formulation based on Archie's Law being the most common for fully saturated (single phase) systems

$$
F_{f}=\frac{1}{a \phi^{m}},
$$

where $a$ is a fitting constant and $m$ is the cementation exponent.

For partially saturated systems, it is common to use the Millington-Quirk formulation (Millington and Quirk, 1961; Moldrup et al., 2000; Sumner, Sumner)

$$
D_{i}^{*}=\phi^{4 / 3} s_{\alpha}^{10 / 3} D_{i}
$$

where the saturation, $s_{\alpha}$, can refer to either the gas or the aqueous phase. 
Multicomponent Diffusion and Electrochemical Migration. Fick's First Law is strictly applicable only in the case of an infinitely dilute solution with uncharged chemical species. In electrochemical systems or systems containing charged species, it is also necessary to consider an electrochemical migration term. While this could be considered as a separate flux on the same level as advection and diffusion, this effect is most commonly related to diffusion of charged species at different rates. The full expression for the migration of a charged species in an electric field is given by Newman and Thomas-Alyea (2004)

$$
\boldsymbol{J}_{i}^{m i g r}=-z_{i} u_{i} F C_{i} \nabla \Phi,
$$

where $z_{i}$ is the charge of the species, $u_{i}$ is its mobility, $F$ is Faraday's constant $(=96,487$ Coulombs/equivalent), and $\Phi$ is the electrical potential. The mobility refers to the average velocity of a species in solution acted upon by a unit force, independent of the origin of the force. The flux of charged species in an electric field gives rise to a current, which can be expressed as

$$
i=F \sum_{i} z_{i} \boldsymbol{J}_{i}
$$

where $i$ is the current density in units of amperes per $\mathrm{m}^{2}$. Expanding Equation (4.20) in terms of the migration term (Equation (4.19)) and the diffusive flux (Equation (4.11)), the current density can be written as

$$
i=-F^{2} \nabla \Phi \sum_{i} z_{i}^{2} u_{i} C_{i}-F \sum_{i} z_{i} \nabla\left(D_{i} C_{i}\right) .
$$

Where no concentration gradients are present, the current is given by the first term on the right hand side

$$
i=-\kappa_{e} \nabla \Phi
$$

where

$$
\kappa_{e}=F^{2} \sum_{i} z_{i}^{2} u_{i} C_{i}
$$

is the conductivity of the solution. Note that using Equation (4.23), it is possible to determine the mobility of an ion by measuring the conductivity of a solution. The mobility in turn can be used to determine the diffusion coefficient for an ion from the Nernst-Einstein equation (Lasaga, 1998; Newman and Thomas-Alyea, 2004)

$$
D_{i}=R T u_{i}
$$

where $R$ is the gas constant and $T$ is the temperature on the Kelvin scale. Rearranging Equation (4.21) to obtain an expression for the gradient in the electrical potential

$$
\frac{\partial \Phi}{\partial x}=-\frac{i}{\kappa_{e}}-\frac{F}{\kappa_{e}} \sum_{i} z_{i} \nabla\left(D_{i} C_{i}\right),
$$

it is apparent that even in the absence of an electrical current, $i$, it is possible to have a gradient in the electrical potential as a result of concentration gradients of charged species. The second term on the right hand side of Equation (4.25) is known as the diffusion potential, which vanishes when 
all of the diffusion coefficients for the charged species are the same. To proceed further, it is useful to define the fraction of the current carried by a species $j$ (or the transference number)

$$
t_{j}=\frac{z_{j}^{2} u_{j} C_{j}}{\sum_{i} z_{i}^{2} u_{i} C_{i}}
$$

which can be used along with the substitution of Equation (4.25) and Equation (4.23) into Equation (4.19) to obtain an expression for the migration flux

$$
\boldsymbol{J}_{j}^{m i g r}=\frac{t_{j} i}{z_{j} F}+\frac{t_{j}}{z_{j}} \sum_{i} z_{i} \nabla\left(D_{i} C_{i}\right)
$$

This equation (still only strictly applicable to relatively dilute solutions) can be used to describe the case where an electrical current is present (e.g., an electrochemical cell), although more commonly in fluid-rock interaction one encounters only the second term. Note that for corrosion problems, the current cannot be neglected to the extent that cathodic and anodic corrosion sites need to be resolved spatially. If the spatial scale of interest is larger than the scales separating the anodic and cathodic sites, an explicit treatment of the electrical current is not needed.

In the absence of a current and advection, the total flux (combining Fickian diffusion and electrochemical migration) is then given by

$$
\boldsymbol{J}_{j}=-\nabla\left(D_{j} C_{j}\right)+\frac{t_{j}}{z_{j}} \sum_{i} z_{i} \nabla\left(D_{j} C_{j}\right)
$$

As noted above, the second term vanishes when all of the diffusion coefficients for the charged species are the same, but since this is in general not the case, this term should normally be retained along with the first term. Note also that the effect of electrochemical migration may be important even in dilute systems.

In the case where a gradient in chemical potential rather than concentration is used, the migration flux (compare to Equation (4.27) in the absence of a current) becomes

$$
\boldsymbol{J}_{j}^{m i g r}=\frac{t_{j}}{z_{j}} \sum_{i} z_{i} \nabla\left(D_{i} \mu_{i}\right)
$$

By writing the chemical potential as (Denbigh, 1981)

$$
\mu_{j}=\mu_{j}^{0}+R T \ln \left(\gamma_{j} C_{j}\right)
$$

where $\gamma_{j}$ is the activity coefficient for the species, and differentiating with respect to $x$, we obtain the following combined expression for the pure diffusive flux and electrochemical migration by making use again of the definition of the ion mobility (Equation (4.10))

$$
\boldsymbol{J}_{j}=-\boldsymbol{\nabla}\left(D_{j} C_{j}\right)-\boldsymbol{\nabla}\left(D_{j} C_{j} \ln \gamma_{j}\right)+\frac{t_{j}}{z_{j}} \sum_{i} z_{i} \boldsymbol{\nabla}\left(D_{i} C_{i}\right)+\frac{t_{j}}{z_{j}} \sum_{i} z_{i} \boldsymbol{\nabla}\left(D_{i} \gamma_{i}\right)
$$

This more general expression, which reduces to Equation (4.28) where gradients in activity coefficients are negligible (for example, for diffusion of a trace species in a strong electrolyte), makes clear that both the diffusive and electrochemical migration fluxes can depend on the activity coefficients for the species. If gradients in one or more activity coefficients are negative, it is possible for "uphill diffusion", in which a species diffuses up its own concentration gradient, to occur (Lasaga, 1998). 


\subsubsection{Data Needs}

For modeling of molecular diffusion in porous media, two kinds of data are needed:

1. Experimental data on the diffusivities of individual ions in aqueous solution;

2. Characterization of the tortuosity of the porous medium under consideration. The tortuosity of the medium may be determined by a number of methods, including transport experiments involving tracers (Navarre-Sitchler et al., 2009), 2) microscopic imaging of the porous medium using synchrotron X-ray or related methods (Navarre-Sitchler et al., 2009), or estimation based on grain size and mineralogy of the materials. In addition, it may be possible to determine tortuosity from effective diffusion coefficients determined in field-scale experiments.

For the diffusivities of individual ions, there are some compilations in the literature (Lasaga, 1998; Steefel and Maher, 2009). While the diffusivity of individual ions could in theory be calibrated from field tests, normally they should be determined independently so that a more accurate determination of the tortuosity can be carried out. 


\section{Biogeochemical Reaction Processes}

A range of biogeochemical reaction processes may be included in the HPC Simulator, including multicomponent aqueous complexation, sorption (including simple linear distribution coefficient, or $\mathrm{Kd}$, and more complex multicomponent and multisite surface complexation and ion exchange models), mineral dissolution and precipitation, and microbially-mediated reactions.

\subsection{Aqueous Complexation}

\subsubsection{Overview}

It is customary to treat the reaction network corresponding to the homogeneous reactions in the aqueous phase as a distinct set of processes taking place within a single aqueous phase (Steefel and MacQuarrie, 1996), although these can also be described within the same more general formalism provided in Section 2 that includes multiphase reactions. This reaction network is sometimes referred to as aqueous complexation, since it involves reactions between individual dissolved species to form complexes.

\subsubsection{Process Model Equations}

Equilibrium Reactions. If we assume that the various aqueous species are in chemical equilibrium, it is possible to reduce the number of independent concentrations, that is, the number that actually need to be solved for. Mathematically, this means that in a system containing $N_{\text {tot }}$ aqueous species, the number of independent chemical components in the system $N_{c}$ is reduced from the total number of species by the $N_{x}$ linearly independent chemical reactions between them (for further discussion, see Aris (1965); Bowen (1968); Hooyman (1961); Kirkner and Reeves (1988); Lichtner (1985); Reed (1982); Van Zeggeren and Storey (1970). This leads to a natural partitioning of the system into $N_{c}$ primary or basis species, designated here as $C_{j}$, and the $N_{x}$ secondary species, referred to as $C_{i}$ (Kirkner and Reeves, 1988; Lichtner, 1985; Reed, 1982). The equilibrium chemical reactions between the primary and secondary species take the form

$$
\mathcal{A}_{i} \rightleftharpoons \sum_{j=1}^{N_{c}} \nu_{i j} \mathcal{A}_{j} \quad\left(i=1, \ldots, N_{x}\right),
$$

where the $\mathcal{A}_{j}$ and the $\mathcal{A}_{i}$ are the chemical formulas of the primary and secondary species respectively and $\nu_{i j}$ is the number of moles of primary species $j$ in one mole of secondary species $i$. It should be noted here that the partitioning between the primary and secondary species is not unique, that is, we can write the chemical reactions in more than one way. The equilibrium reactions provide an algebraic link between the primary and secondary species via the law of mass action for each reaction

$$
C_{i}=K_{i}^{-1} \gamma_{i}^{-1} \prod_{j=1}^{N_{c}}\left(\gamma_{j} C_{j}\right)^{\nu_{i j}} \quad\left(i=1, \ldots, N_{x}\right),
$$

where $\gamma_{j}$ and $\gamma_{i}$ are the activity coefficients for the primary and secondary species respectively, and the $K_{i}$ are the equilibrium constants of the reaction given in Equation (5.2), written here as the 
destruction of one mole of the secondary species. Equation (5.2) implies that the rate of production of a primary component $j$ due to homogeneous reactions, $R_{j}^{a q}$, can be written in terms of the sum of the total rates of production of the secondary species (Kirkner and Reeves, 1988)

$$
R_{j}^{a q}=-\sum_{i=1}^{N_{x}} \nu_{i j} I_{i}^{a q}
$$

where $I_{i}^{a q}$ are the reaction rates of the secondary species in the aqueous phase. Equation (5.3) suggests that one can think of a mineral dissolving, for example, as producing only primary species which then equilibrate instantly with the secondary species in the system. Using Equation (5.3) and neglecting transport for the sake of simplicity here, the rates of the equilibrium reactions can be eliminated (Steefel and MacQuarrie, 1996)

$$
\frac{\partial}{\partial t}\left[\phi s_{w} \rho_{w}\left(C_{j}+\sum_{i=1}^{N_{x}} \nu_{i j} C_{i}\right)\right]=R_{j}^{\text {min }}\left(j=1, \ldots, N_{c}\right)
$$

where $s_{w}$ and $\rho_{w}$ refer to the saturation and mass density of the aqueous phase, respectively. Note that only the term $R_{j}^{\text {min }}$ remains on the right hand side of Equation (5.4) because we have assumed that they are the only kinetic reactions.

Definition of a Total Concentration. If a total concentration, $\Psi_{j}$, is defined as (Kirkner and Reeves, 1988; Lichtner, 1985; Reed, 1982)

$$
\Psi_{j}=C_{j}+\sum_{i=1}^{N_{x}} \nu_{i j} C_{i}
$$

then the governing differential equations can be written in terms of the total concentrations in the case where only aqueous (and therefore mobile) species are involved (Kirkner and Reeves, 1988)

$$
\frac{\partial}{\partial t}\left(\phi s_{w} \rho_{w} \Psi_{j}\right)+\nabla \cdot\left[\phi s_{w} \rho_{w} v_{w} \Psi_{j}-D \nabla\left(\phi s_{w} \rho_{w} \Psi_{j}\right)\right]=R_{j}^{\min } \quad\left(j=1, \ldots, N_{c}\right),
$$

where $v_{w}$ is the velocity of the aqueous phase. As pointed out by Reed (1982) and Lichtner (1985), the total concentrations can usually be interpreted in a straightforward fashion as the total elemental concentrations (e.g., total aluminum in solution), but in the case of $H^{+}$and redox species, the total concentration has no simple physical meaning and the total concentrations may take on negative values. Such quantities, however, do appear occasionally in geochemistry, the best example of which is alkalinity. In fact, the alkalinity (which may take on negative values) is just the negative of the total $\mathrm{H}^{+}$concentration where $\mathrm{CO}_{2}(\mathrm{aq})$ or $\mathrm{H}_{2} \mathrm{CO}_{3}$ is chosen as the basis species for the carbonate system.

Note that the total concentration is generally only a useful concept computationally where equilibrium reactions allow the definition of secondary species described with Equation (5.3). In the case where the reactions among aqueous species are described kinetically, then the various aqueous complexes cannot be eliminated algebraically and they have to be solved for individually. 
Kinetic Aqueous Reactions. If the aqueous phase reactions are not sufficiently fast for a given time scale of interest that they reach equilibrium, then they must be treated kinetically by solving an ordinary differential equation. A convenient way to represent the reactions is with a Transition State Theory (TST) type of rate law (Aagaard and Helgeson, 1982; Lasaga, 1981, 1984)

$$
I_{j}^{a q}=k_{+}^{a q}\left(\frac{Q^{a q}}{K^{a q}}\right) \prod a_{i}^{n},
$$

where $k_{+}^{a q}$ is the rate constant for the aqueous reaction, $Q^{a q}$ is the ion activity product, $K^{a q}$ is the corresponding equilibrium constant, and $a_{i}$ are the activities of the species affecting the rate far from equilibrium raised to the power $n$.

Alternatively, the reactions can be considered as completely irreversible, in which case there is no back reaction. A good example is radioactive decay. The reactions are assumed to take the form

$$
I_{j}^{a q}=k_{+}^{a q} \prod a_{i}^{n}
$$

and are therefore similar to the TST form except that a dependence on the saturation state is missing.

\subsection{Aqueous Activity Coefficients}

\subsubsection{Overview}

This Toolset includes models for thermodynamic activity coefficients in aqueous solutions. Multiple models, each with its own set of parameters and limitations, will be provided. In general, the toolset user must choose one such model to use in a given modeling application. In setting up to run the application, the user must ensure that a matching database with the requisite model-specific parameters is provided to support the run.

A key solution parameter associated with aqueous species activity coefficients is the ionic strength, defined as

$$
\bar{I}=\frac{1}{2} \sum_{i} m_{i} z_{i}^{2} .
$$

Here $m_{i}$ denotes the molal concentration (molality) of the $i^{\text {th }}$ solute species and $z_{i}$ denotes its electrical charge number. Activity coefficients of charged solute species include a functional dependence on the ionic strength. The exact nature of this dependence depends on the specific model. Activity coefficient models can be classified as to the upper limit of ionic strength to which a given model provides generally satisfactory results. For the most part, there are two kinds of such models. Low ionic strength models are generally usable up to an ionic strength of more or less 1 molal. Examples include the Davies equation and the B-dot equation. These models are based on simple equations and have a relatively small number of associated parameters. High ionic strength models are usable to very high ionic strength ( $>20$ molal). The highest values of ionic strength normally seen are limited by the solubilities of highly soluble salts, such as calcium chloride and calcium nitrate. Examples of high ionic strength models include Pitzer's equations and Extended UNIQUAC. High ionic strength models have more complex equations and require substantially 
more parameters than low ionic strength models. They are most likely to be applied to problems in which the ionic strength is higher than 1 molal. At low ionic strength, it is generally preferable to use a low ionic strength model, because the number of required parameters is smaller. In many instances, there will be sufficient supporting data available to support the use of low ionic strength models for large numbers of chemical components and species, but that may not be the case for the high ionic strength models. There are activity coefficient models that extend to intermediate ionic strength (4-6 molal). The NEA SIT model is an example. These models tend to be intermediate in equation complexity and number of required parameters. They have not received much attention to date in modeling geochemically complex systems.

It is noted that low ionic strength models are sufficient for many EM applications. Hanford tanks and the WIPP site pose notable exceptions, requiring the use of high ionic strength models. There may be other instances in which the use of low ionic strength models may be inappropriate.

There are two kinds of activity coefficients that a model should be able to provide. The first is the molal activity coefficient of a solute species (denoted by $\gamma_{i}$ ). This is subsequently used to compute the thermodynamic activity of the corresponding species (denoted by $a_{i}$ ). The activity of a species is obtained by multiplying the molality (molal concentration) of the species (denoted by $m_{i}$ ) by the molal activity coefficient:

$$
a_{i}=m_{i} \gamma_{i}
$$

This activity is then used in various equations describing thermodynamic equilibrium and chemical kinetics.

The second kind of activity coefficient, the rational activity coefficient, pertains to the solvent, water $(w)$. Its activity coefficient is denoted by $\lambda_{w}$ to emphasize that it is different in kind: it is a mole fraction activity coefficient. The thermodynamic activity of water $\left(a_{w}\right)$ is obtained by multiplying the mole fraction of water $\left(X_{w}\right)$ by the activity coefficient of water:

$$
a_{w}=X_{w} \lambda_{w}
$$

The activity of water is also different in kind from the activity of a solute species (a mole fraction activity as opposed to a molal activity). In treating the thermodynamics of aqueous electrolyte solutions, the activity of water and the activity of a solute species are almost always treated as described above.

Activity coefficients are generally first calculated in logarithmic form (e.g., $\ln \gamma_{i}$ or $\log \gamma_{i}$ ). In practical usage, activity coefficients are used most often used in base-10 logarithmic form, being converted from natural logarithm form as necessary. The conversion is illustrated by

$$
\log \gamma_{i}=\frac{\ln \gamma_{i}}{\ln (10)}
$$

The conversion factor $\ln (10)$ is approximately equal to 2.303 , and this value often appears in equations in the literature in place of the exact factor. The approximate value should not be used in this toolset, as it is insufficiently precise for accurate work. Instead the value should be calculated using the same floating-point precision that will be used to calculate activity coefficients. This is most efficiently done by calculating the value once and then storing it for subsequent use. It is noted that "log" is somewhat ambiguous, in that the literature contains examples of it being used for both 
natural and base-10 logarithms. In the present description given in this section (Section 5.2), it will always refer to the base-10 logarithm.

Activity coefficient model equations ideally satisfy thermodynamic consistency relations. The value of consistency lies in allowing the possibility of accuracy at higher ionic strengths. Low ionic strength models typically include inconsistent equations, but the numerical consequences of the inconsistencies tend to be acceptable in the range of applicability of these models. For electrolyte solutions, Wolery (1992b) presents equations and methods for ensuring the development of consistent equations and for testing the consistency of existing sets of equations. The easiest means of testing for consistency is to use the cross-differentiation rule, which takes the following forms for solute-solute and solvent-solute pairs:

$$
\begin{aligned}
\frac{\partial \ln \gamma_{j}}{\partial m_{i}} & =\frac{\partial \ln \gamma_{i}}{\partial m_{j}} \\
N_{w}^{k g} \frac{\partial \ln a_{w}}{\partial m_{i}} & =-1-\sum_{j} m_{j} \frac{\partial \ln \gamma_{i}}{\partial m_{j}}
\end{aligned}
$$

where $i$ and $j$ denote different solute species and $N_{w}^{k g}$ is the number of moles of water in a $1 \mathrm{~kg}$ mass (approximately 55.51).

\subsubsection{The Debye-Hückel Equations}

Activity coefficient model equations for electrolyte solutions generally include some type of DebyeHückel term to represent the effects of long-range electrical forces. The most common representation is based on the "extended" Debye-Hückel equation, which for the activity coefficient of an ionic solute species is given by

$$
\log \gamma_{i}=-A_{\gamma, 10} z_{i}^{2}\left(\frac{\sqrt{\bar{I}}}{1+b \sqrt{\bar{I}}}\right) .
$$

Here $A_{\gamma, 10}$ is the Debye-Hückel " $A$ " parameter for the activity coefficient (hence the subscript " $\gamma$ "), modified for consistency with the base-10 logarithmic activity coefficient on the left-hand-side of the equation (hence the additional subscript, "10"). To assist in avoiding potential confusion, $A_{\gamma, 10}$ should have a value of 0.5114 at $25^{\circ} \mathrm{C}$ and 1.013 bar pressure. The parameter " $b$ " is conceptually the product of the Debye-Hückel " $B$ " parameter for the activity coefficient $\left(B_{\gamma}\right)$ and a length that corresponds to either the diameter of the ion in question or a characteristic distance of closest approach to itself or any other ion in solution. Practical models treat this in various ways. Some assign a constant value, typically 1.0, 1.2, or 1.5. Others use the product of $B_{\gamma}$ (which has a known temperature and pressure dependence) and some sort of length parameter.

The equation for the activity of water corresponding to the extended Debye-Hückel equation is

$$
\log a_{w}=\frac{1}{N_{w}^{k g}}\left(-\frac{\sum_{i} m_{i}}{\ln (10)}+\frac{2}{3} A_{\gamma, 10} \bar{I}^{3 / 2} \varsigma(b \sqrt{\bar{I}})\right) .
$$

where the summation over molalities spans all solute species (all aqueous species except the solvent), and the function $\varsigma(\mathrm{x})$ in Equation (5.16) is given by

$$
\varsigma(x)=\frac{3}{x^{3}}\left(1+x-\frac{1}{1+x}-2 \ln (1+x)\right),
$$


where $x$ serves the purpose of a generic variable. If the activity coefficient of water is desired, it can be obtained from the relation

$$
\log \lambda_{w}=\log a_{w}-\log \left(X_{w}\right)
$$

where the mole fraction of water is given by

$$
X_{w}=\frac{N_{w}^{k g}}{N_{w}^{k g}+\sum_{i} m_{i}} .
$$

The activity of water is closely related to the osmotic coefficient, $\varphi$ :

$$
\varphi=-\left(\frac{N_{w}^{k g}}{\sum_{i} m_{i}}\right) \ln a_{w} .
$$

All forms of the extended Debye-Hückel equation are consistent with the Debye-Hückel Limiting Law (DHLL):

$$
\lim _{\bar{I} \rightarrow 0} \log \gamma_{i}=-A_{\gamma, 10} z_{i}^{2} \sqrt{\bar{I}}
$$

The limiting law is a critical feature describing the behavior of ionic activity coefficients in the range of very low ionic strength. The ionic activity coefficient plunges rapidly from unity as ionic strength increases from zero. There is no comparable limiting relation for the activity of water, due to the compositional dependence on both the ionic strength and the sum of solute molalities.

In general, the extended Debye-Hückel equation is not useful for significant practical modeling, as it is accurate only in very dilute aqueous solutions. If only monovalent ions are present, it may be useful for $\bar{I}<0.1$ molal. In the presence of higher valence ions, the maximum range becomes more compressed. Most practical models therefore extend the "extended" Debye-Hückel equation by adding additional terms or otherwise adding to the mathematical complexity, in the process introducing more model parameters.

The activity coefficient models that will be available in this toolset include the Davies equation, the B-dot equation, Pitzer's equations, Extended UNIQUAC, and NEA-SIT. The models are first addressed, followed by the discussion on rescaling the activity coefficients.

\subsubsection{The Davies Equation}

The Davies (1962) equation is a commonly used at low ionic strength (less than about 1 molal) model. The activity coefficient of an aqueous solute species is given by

$$
\log \gamma_{i}=-A_{\gamma, 10} z_{i}^{2}\left(\frac{\sqrt{\bar{I}}}{1+\sqrt{\bar{I}}}-d \bar{I}\right) .
$$

Here $d$ is a constant, either 0.2 as in EQ3/6 (Wolery, 1992a) or 0.3 as in PHREEQC (Parkhurst and Appelo, 1999). If the " $d \bar{I}$ " part is dropped, this equation reduces to the extended Debye-Hückel form with $b$ set to unity. It can be shown that the full equation satisfies the solute-solute-form of the cross-differentiation rule. 
For the activity coefficient of water, the matching equation used in EQ3/6 for the activity of water is

$$
\log a_{w}=\frac{1}{N_{w}^{k g}}\left(-\frac{\sum_{i} m_{i}}{\ln (10)}+\frac{2}{3} A_{\gamma, 10} \bar{I}^{\frac{3}{2}} \varsigma(\sqrt{\bar{I}})-d A_{\gamma, 10} \bar{I}^{2}\right) .
$$

where all parameters and the $\varsigma(x)$ function have been previously introduced (See Section 5.2.2). This equation is a corrected version of that given by Wolery (1992a) (Equation 86 in that document). Here a factor of 2 in the " $d$ " term has been removed, and $d$ substitutes for the original constant value of 0.2 . This equation is quasi-consistent with the equation for the activity coefficient of a solution species, in the sense that the solvent-solute form of the cross-differentiation rule is satisfied for the case of a pure solution of a uni-univalent electrolyte, such as sodium chloride. It does not satisfy this rule in the general case.

The equation used by PHREEQC is symbolically equivalent to

$$
a_{w}=1-\frac{1}{N_{w}^{k g}} \sum_{i} m_{i} .
$$

As given by the source (Parkhurst and Appelo, 1999, p. 17), the factor $1 / N_{w}^{k g}$ is replaced by a constant value of 0.017 , which is rather approximate, and the molality is replaced by the mole number divided by the number of $\mathrm{kg}$ of solvent water (this substitution is exact). This equation is based on ignoring the activity coefficient of water and replacing the mole fraction with a limiting approximation of itself. Hence, the activity of water is given in direct form, rather than logarithmically.

For the present toolset, it is recommended that the Davies model be implemented as two options, one (Davies-EQ3/6) consistent with the implementation in EQ3/6, the other (Davies-PHREEQC), with PHREEQC. This will permit direct comparison with both codes.

The Davies equation predicts a unit activity coefficient for electrically neutral solute species. This is known to be generally inaccurate, as the activity coefficients of non-polar neutral solutes such as $\mathrm{O}_{2}(\mathrm{aq})$ and $\mathrm{N}_{2}(\mathrm{aq})$ should increase with ionic strength (the "salting out" effect), while the activity coefficients of polar species such as $\mathrm{CaSO}_{4}(\mathrm{aq})$ and $\mathrm{MgSO}_{4}(\mathrm{aq})$ should decrease ("salting-in").

In practice, the Davies equation is mainly used for low temperatures (near $25^{\circ} \mathrm{C}$ ) and near-atmospheric pressures. The $A_{\gamma, 10}$ parameter has temperature and pressure dependence. As long as this is accounted for, the Davies equation model could be applied in principle at higher temperatures and pressures. However, it needs to be kept in mind that the 0.2 constant was obtained by fitting data to solutions for temperature near $25^{\circ} \mathrm{C}$ and for atmospheric pressure. The accuracy of the model is therefore likely to deteriorate at higher temperatures and pressures.

\subsubsection{The B-dot Equation}

The B-dot equation of Wolery (1969) is an alternative low ionic strength model. The activity coefficient of a solute species is given by

$$
\log \gamma_{i}=-\frac{A_{\gamma, 10} z_{i}^{2} \sqrt{\bar{I}}}{1+\stackrel{\circ}{a}_{i} B_{\gamma} \sqrt{\bar{I}}}+\dot{B} \bar{I}
$$

where $\stackrel{\circ}{a}_{i}$ is the diameter of the $i^{\text {th }}$ solute species, $B_{\gamma}$ is the Debye-Hückel B parameter for the activity coefficient, and $\dot{B}$ is the "B-dot" parameter. Removing the $\dot{B} \bar{I}$ term and setting $\stackrel{a}{a} B_{\gamma}$ to 
unity, this equation reduces to the Davies equation with the $d \bar{I}$ term omitted. Comparison with the Davies equation brings up two points. The first is that the B-dot model has more parameters. The $B_{\gamma}$ parameter appears, and each solute species has an assigned diameter. The "B-dot" parameter itself is an additional parameter.

It can be shown that the B-dot equation does not satisfy the solute-solute form of the crossdifferentiation rule. There is an issue with the first term on the right hand side in that the rule can only be satisfied if all aqueous ions have the same diameter. There is an issue with the second term in that the rule is only satisfied if the charge number squared is the same for all ions, as would be the case for example in a pure sodium chloride solution.

For an electrically neutral species, the B-dot equation reduces to

$$
\log \gamma_{i}=\dot{B} \bar{I}
$$

As the $\dot{B}$ parameter is generally assigned a positive value, this would provide for some measure of "salting-out." By tradition, however, the B-dot equation is not applied to neutral solute species, and it will not be so applied in the present toolset. For non-polar neutral species, the common practice is to assign an approximation for the activity coefficient of $\mathrm{CO}_{2}$ (aq) in otherwise pure sodium chloride solution of the same ionic strength. The approximation used in EQ3/6 (based on S. E. Drummond (1981), and which will be adopted for the present toolset) is

$$
\ln \gamma_{i}=\left(C+F T+\frac{G}{T}\right) I-(E+H T)\left(\frac{\bar{I}}{\bar{I}+1}\right) .
$$

Here $\mathrm{T}$ is the absolute temperature and $\mathrm{C}=-1.0312, \mathrm{~F}=0.0012806, \mathrm{G}=255.9, \mathrm{E}=0.4445$, and $\mathrm{H}=-0.001606$. Note that the result is presented in terms of the natural logarithm. For a polar aqueous species, the EQ3/6 practice (which will be adopted in the present toolset) is to use

$$
\log \gamma_{i}=0
$$

Because different equations are used for electrically neutral solute species than for ionic species, there is necessarily an additional set of violations of the solute-solute cross-differentiation rule.

For the activity of water, the B-dot model as implemented in EQ3/6 (and recommended for the present toolset) is to use the equation

$$
\log a_{w}=\frac{1}{N_{w}^{k g}}\left(-\frac{\sum_{i} m_{i}}{\ln (10)}+\frac{2}{3} A_{\gamma, 10} \bar{I}^{\frac{3}{2}} \sigma\left(\stackrel{\circ}{a} B_{\gamma} \sqrt{\bar{I}}\right)-\dot{B} \bar{I}^{2}\right) .
$$

All the parameters here have been introduced previously, except for the unsubscripted $\stackrel{\circ}{a}$, which is conceptually an effective solute species diameter. In practice, this is assigned a constant value of 4.0 angstroms.

The above equation for the activity of water is quasi-consistent with the solvent-solute form of the cross-differentiation rule. The term containing the effective solute diameter leads to inconsistency unless every ionic solute has a matching diameter value. The term containing $\dot{B}$ leads to inconsistency unless the solution is a pure solution of a uni-univalent electrolyte such as sodium chloride. 
The thermodynamic inconsistencies noted above introduce some level of inaccuracy into the model, tending to negate the improvement that might be expected by introducing more parameters (e.g., a diameter value specific to each solute species). Thus, for temperature near $25^{\circ} \mathrm{C}$ and nearatmospheric pressure, the B-dot model is probably as good as the Davies equation model.

The B-dot model does have an advantage over the Davies equation model in that it is better parameterized to cover a wide range of temperature and pressure. In addition to $A_{\gamma, 10}$, the $B_{\gamma}$ and $\dot{B}$ parameters are treated as functions of temperature and pressure. The $A_{\gamma, 10}$ and $B_{\gamma}$ parameters have values derived from pure theory (and models for pure water properties). The $\dot{B}$ parameter is obtained by fitting to data for pure sodium chloride solutions. The ion size parameters are treated as constant with respect to temperature and pressure.

In regard to solute species, diameters are only necessary for ionic species. Some means needs to be provided to specify (as on a supporting thermodynamic data file) whether a neutral species is to be treated as non-polar or polar. All the necessary information could be folded into a diameter array or equivalent structure, in which the values in the case of neutral species would not be actual diameters, but code values specifying non-polar or polar type. However, a separate flagging structure should be utilized, as the variable type can then be something more appropriate (integer or logical) than the floating point necessary for actual diameters. Also, the structure for diameters would then be free to include diameters for neutral solute species. Although such diameters are not be used in the B-dot model, they might be usable in other models. 


\subsubsection{Pitzer's Equations}

At the present time, models of activity coefficients in high ionic strength solutions are most commonly based on Pitzer's equations (Pitzer, 1973, 1991). The basic approach is to extend a DebyeHückel representation of long-range electrical forces with a virial (interaction coefficient) expansion, accounting for interactions among solute species pairs and triplets. A characteristic of the Pitzer equations is an ionic strength dependence of the pair interaction parameters. Modern forms of the Pitzer equations also include a theoretical accounting of higher-order electrical interactions (Harvie et al., 1984; Pitzer, 1975), which is described in terms of pair interactions for certain kinds of pairs of solute ions. However, the pair and triplet interactions are mainly described by parameters obtained by fitting to experimental data, as for osmotic coefficients of solutions of single aqueous electrolytes and pairs of aqueous electrolytes sharing a common ion, and for solubilities of various salt minerals.

The Pitzer equations themselves only provide a mathematical framework. That exists in two principal forms, the original one based on expansion in molalities, and a later one based on expansion in mole fractions (Pitzer and Simonson, 1986). The latter approach solves some problems in the region in which solutions become so concentrated that solvent water is not abundant relative to solute species. It is less commonly applied and fitted parameter data are consequently less abundant. It is likely to be necessary only in limited circumstances, such as when there is a co-solvent such as ethanol which can be much more abundant than water or where there is a solution that is effectively a slightly hydrous molten salt. The mole fraction form might be considered for future addition to the toolkit if future applications warrant. At the present time only the molal form will be addressed for inclusion as an option in the toolkit.

The Pitzer equations are derived using a mathematical approach that, in the absence of mistakes in partial differentiation, guarantees that the relevant consistency relations are satisfied (see Wolery 1992b). Because these equations are a basis for high ionic strength modeling, this consistency is a necessary (but not necessarily sufficient) component to achieving accurate results.

Because the Pitzer approach is based on fitting experimental data, a model per se only follows when the equations are fit to experimental data. There is some variation in Pitzer-based models owing to choices of experimental datasets and dataset weighting. Some variation is associated with the exact choice of value for the Debye-Hückel $A_{\phi}$ parameter (which is related to, but not the same as, the $A_{\gamma, 10}$ parameter discussed previously). This occurs because the values of fitted parameters depend on the value of $A_{\phi}$ used in the fitting. A second source of variation comes from the assumed set of solute species present. For example, a calcium chloride solution can be modeled assuming only $\mathrm{Ca}^{2+}$ and $\mathrm{Cl}^{-}$, or with additional species such as $\mathrm{CaCl}^{+}$or $\mathrm{CaCl}_{2}(\mathrm{aq})$. Additional species present additional interaction parameters, and require adjustments to the other relevant parameters not involving the additional species (e.g., including $\mathrm{CaCl}^{+}$requires adjusting the pair interaction parameters for $\mathrm{Ca}^{2+}$ and $\mathrm{Cl}^{-}$from the values appropriate when not including the ion pair). In general, the practice of developing Pitzer models (and other high ionic strength models) has been to minimize the number of species considered. Thus, typical high ionic strength models do not include many of the ion pair and complex species that are often present in low ionic strength models. A third source of variation is that in some instances the available experimental data are insufficient to obtain a unique fitting to the set of relevant fitting parameters. In such cases, some parameters may be arbitrarily assigned a value of zero. This is especially likely when ion 
pair and complex species are included in the model. An additional factor in variation has to do with the description of the temperature dependence of the model parameters, as different more or less arbitrary functions are used for this purpose.

The more extensive Pitzer model databases are divided into two types. Models which only pertain to a temperature of $25^{\circ} \mathrm{C}$ are almost all built using as a core the model of Harvie et al. (1984) for the $\mathrm{Na}-\mathrm{K}-\mathrm{Mg}-\mathrm{Ca}-\mathrm{H}-\mathrm{Cl}-\mathrm{SO}_{4}-\mathrm{OH}-\mathrm{HCO}_{3}-\mathrm{CO}_{3}-\mathrm{CO}_{2}-\mathrm{H}_{2} \mathrm{O}$ system at this temperature. The Harvie model is notable because it provided the first integrated description in the Pitzer framework of activity coefficients and mineral solubilities for a reasonably large geochemical system. Prior works had mainly focused on describing measurements of the osmotic coefficient (related to the activity of water) in pure liquid systems, or touched on the solubility of minerals in more limited systems. The Harvie et al. (1984) model is often supplemented with the borate extension of Felmy and Weare (1986). Various extant low temperature databases (including the WIPP thermodynamic database) include other extensions.

Some Pitzer databases extend to higher temperature. Most of these include as a core the model of Greenber and Moller (1989) for the system Na-K-Ca-Cl-SO ${ }_{4}-\mathrm{H}_{2} \mathrm{O}$, which covers the range 0$250^{\circ} \mathrm{C}$. This work also addresses mineral solubilities. The model of Pabalan and Pitzer (1987) for the system Na-K-Ca-Mg-Cl-SO $\mathrm{S}_{4}-\mathrm{OH}-\mathrm{H}_{2} \mathrm{O}$ extending to high temperature $\left(\sim 300^{\circ} \mathrm{C}\right.$ in at least some parts) also directly addresses mineral solubilities and is equally notable. Various extant high temperature Pitzer databases include one or both of these (e.g., the Yucca Mountain Project Pitzer database, a Hanford tanks database), usually supplemented with additional data.

A point to be made is that combining data from different sources may lead to inconsistencies. In constructing and maintaining databases, it may be necessary to at least evaluate if not correct inconsistencies. In some instances, the data must be reformulated to follow a common format, such as a common temperature function or for consistency with say a slightly different value of the $A_{\phi}$ parameter.

In general, there is a small inconsistency between low temperature $\left(25^{\circ} \mathrm{C}\right)$ and high temperature Pitzer databases in that virtually all low temperature work uses the $A_{\phi}$ value of 0.392 originally used by Harvie et al. (1984) (who incorrectly reported a value of 0.39, see Plummer et al. (1988) or Plummer and Parkhurst (1990)). Temperature functions describing $A_{\phi}$ generally yield a value close to 0.391 when evaluated at $25^{\circ} \mathrm{C}$. It has been shown (Plummer and Parkhurst, 1990; Plummer et al., 1988) that small differences in this parameter can be significant in the computation of mineral solubilities. The parameter itself appears not only in the Debye-Hückel part of the model equations, but also in the theory-based part describing higher-order electrical interactions.

The Pitzer models addressed here only describe activity coefficients and their temperature dependence. It is noted that volumetric property models based on the Pitzer framework (e.g. Kumar, 1986; Monnin, 1989) do exist. These models can provide the solution density as a function of detailed composition. The data for such models is in principal the same as that required to calculate the pressure dependence of the activity coefficients. Similarly, enthalpic properties are also treatable within the larger Pitzer framework (cf. Pitzer, 1991). However, there are relatively few applications addressing the volumetric and enthalpic properties, and most modeling codes ignore them or use simplified approximations for a few key necessary items (e.g., estimate the density of brine by substituting the density of a sodium chloride solution having the same total dissolved salt content). 
The ideas behind Pitzer's equations are relatively simple. The mathematical development (Pitzer, 1973, 1991) begins with writing an expression for the excess Gibbs energy. This consists of a Debye-Hückel term and a virial expansion in the mole numbers of solute species that is truncated at third order. The expansion includes only second and third order terms; first order terms are excluded because the excess Gibbs energy is an extensive thermodynamic quantity and so must be described by a zero-th order homogeneous equation. The expansion includes second and third order virial coefficients (respectively represented by $\lambda_{i j}$ and $\mu_{i j k}$, where $i, j$, and $k$ each span the set of aqueous solute species). In the general form, a species may be repeated in a pair or triplet subscripting. There are no virial terms or coefficients involving the solvent, water, because the excess Gibbs energy must be zero when the solution consists of pure water. Expressions for the solute activity coefficient and the osmotic coefficient (which is closely related to the activity of water, see below) are derived using pertinent partial differentiation (Pitzer, 1973, 1991; Wolery, 1992b).

A key aspect of the Pitzer approach is that second order virial coefficients for ionic interactions are treated as functions of ionic strength. This is responsible for much of the success of the approach compared to prior approaches incorporating virial expansion. A distinguishing feature of the Pitzer model is its usage of the Debye-Hückel-osmotic (DHO) model as opposed to the more familiar Debye-Hückel-charging (DHC) model, which is incorporated for example in the Davies (1962) equation and the B-dot equation (Wolery, 1969). This substitution probably has little to do with the success of the Pitzer model, but it is a characteristic. Pitzer (1975) modified the original 1973 model by including a theoretical description of higher order electrostatic effects. This was incorporated in the form of adding a theoretical component to the pair interactions for ions of the same charge sign but different charge number (e.g., $\mathrm{Na}^{+}, \mathrm{Ca}^{2+}$ ). All modern work with Pitzer's equations includes this modification. The modification includes a representation of the $\mathrm{J}(\mathrm{x})$ and $\mathrm{J}$ '(x) functions (see below), which are defined by integral equations and consequently are represented by numerical approximations for the sake of rapid, practical calculation. Pitzer (1975) presented some suggested formulas. However, all modern work utilizes the approximation developed by Harvie (1981). The use of older approximations may lead to noticeable inconsistencies.

The actual practical equations for the Pitzer model are fairly complex. Much of the complexity results from the fact that electroneutrality requires that many of the theoretical virial coefficients are only observable in certain combinations. These combinations have been effectively defined as the set of practical interaction coefficients, and are mostly represented by additional symbols. The original $\lambda_{i j}$ and $\mu_{i j k}$ coefficients only carry through for some species combinations involving neutral solute species. The various types of practical interaction coefficients give rise to different types of summations in the equations for solute activity coefficients and for the osmotic coefficient (or the activity or activity coefficient of water).

The Pitzer notation deserves some comment, as it may otherwise be confusing. There are several instances in which superscript symbols are used as labels, not exponents. Examples include the superscript $\phi$ in the interaction coefficient $C_{c a}^{\phi}$ and the pre-superscript $E$ in the interaction coefficient (actually function) ${ }^{E} \theta_{c c^{\prime}}$. Although $\phi$ is used as a label, it is also used to symbolize the osmotic coefficient. The usage as label traces to actual and historical ties to equations for or closely related to the osmotic coefficient.

The coding of Pitzer's equations poses its own difficulties. This task is much more difficult than 
coding the Davies equation or the B-dot model. One fundamental issue is how to store the relevant data. Consider a parameter that goes with a cation-anion pair. Should the data be stored in a 2-D matrix (cations by anions)? Or should each cation-anion pair for which non-zero data exists be treated as the fundamental entity, and relevant lists be constructed identifying the pair members and giving the corresponding parameter values? This issue must be addressed multiple times, and there are multiple types of interaction coefficients and associated parameters. The types of data are associated with certain summations (singe, double, and triple) in the equations for the activity coefficients, and the issue of data storage is necessarily tied to the most efficient means of evaluating the summations. This is not trivial. Also, because of the complexity, it is difficult to avoid errors. Consequently, careful code-to-code validation will be necessary.

The types of Pitzer model data are listed in Tables 2-3. Table 2 summarizes the practical interaction coefficients. All of these coefficients may potentially be treated as functions of temperature and pressure (see below). The coefficients $\beta_{c a}^{(0)}, \beta_{c a}^{1}, \beta_{c a}^{2}, C_{c a}^{\phi}$ describe cation-anion interactions. $\beta_{c a}^{2}$ is only used for certain cation-anion pairs (as will be noted below). These coefficients are generally obtained by regressing osmotic coefficient data for binary systems consisting of the solvent water and a single aqueous electrolyte, such as $\mathrm{NaCl}$. The coefficients ${ }^{S} \theta_{c c^{\prime}}$ and ${ }^{S} \theta_{a a^{\prime}}$ respectively describe (in part, see below) the interactions between a cation $(c)$ and a different cation ( $c$ '), or an anion $(a)$ and a different anion $\left(a^{\prime}\right)$. These coefficients are most often tabulated omitting the presuperscript $S$ (for "short range"). The coefficients $\psi_{c c^{\prime} a}$ and $\psi_{c c^{\prime} a}$ respectively describe interactions among a cation, a different cation, and an anion, and an anion, a different anion, and a cation. Other parameters involve neutral solute species $(n)$. The coefficients $\lambda_{n c}$ and $\lambda_{n a}$ respectively describe neutal-cation and neutral-anion second order interactions. These represent the curious case of theoretical $\lambda$ coefficients that cannot be combined into an equivalent practical interaction coefficient, but which still cannot be determined one in the absence of the other. It is necessary to establish some convention for their practical use. Harvie et al. (1984) adopted the convention that

$$
\lambda_{n, H^{+}}=0 .
$$

The usual alternative (e.g. Clegg and Brimblecombe, 1990) is

$$
\lambda_{n, C l^{-}}=0 .
$$

Most Pitzer models follow this, but not all. For the present toolkit, the Harvie et al. (1984) convention is preferred. The implementation of such a convention would be in a supporting data file, not in software. Therefore, the software should be capable of following either convention.

The coefficient $\sigma_{n c a}$ describes interactions among a neutral, a cation, and an anion. The coefficients $\lambda_{n n}, \mu_{n n n}$ respectively describe second and third order interactions involving a neutral and itself. The coefficients $\lambda_{n n^{\prime}}, \mu_{n n n^{\prime}}, \mu_{n^{\prime} n^{\prime} n}$ describe second and third order interactions among a neutral $(n)$ and a different neutral ( $\left.n^{\prime}\right)$.

Coefficients of the form $\mu_{n n c}$ and $\mu_{n n a}$ describe third order interactions among a neutral and a cation or anion respectively (cf. Clegg and Brimblecombe, 1990). They bear a certain analogy to the coefficients $\lambda_{n c}$ and $\lambda_{n a}$ in that an analogus convention is required. The preferred convention for the present toolkit is analogous to that of Harvie et al. (1984) for those $\lambda$ coefficients:

$$
\mu_{n, n, H^{+}}=0
$$


Table 2: Key Pitzer interaction coefficients.

\begin{tabular}{ll} 
Species Combination & Interaction Coefficients \\
\hline \hline Cation-anion $(c a)$ & $\beta_{c a}^{(0)}, \beta_{c a}^{1}, \beta_{c a}^{2}, C_{c a}^{\phi}$ \\
\hline Cation-different cation $\left(c c^{\prime}\right)$ & ${ }^{S} \theta_{c c^{\prime}}$ (usually tabulated as ' $\left.\theta_{c c^{\prime}}{ }^{\prime}\right)$ \\
\hline Anion-different anion $\left(a a^{\prime}\right)$ & ${ }^{S} \theta_{a a^{\prime}}$ (usually tabulated as ' $\left.\theta_{a a^{\prime}}{ }^{\prime \prime}\right)$ \\
\hline Cation-different cation-anion $\left(c c^{\prime} a\right)$ interactions & $\psi_{c c^{\prime} a}$ \\
\hline Anion-different anion-cation $\left(a a^{\prime} c\right)$ interactions & $\psi_{c c^{\prime} a}$ \\
\hline Neutral-cation $(n c)$ & $\lambda_{n c}$ \\
\hline Neutral-anion $(n a)$ & $\lambda_{n a}$ \\
\hline Neutral-cation-anion $(n c a)$ & $\sigma_{n c a}$ \\
\hline Neutral-same neutral-cation & $\mu_{n n c}$ \\
\hline Neutral-same neutral-anion & $\mu_{n n a}$ \\
\hline Neutral-same neutral $(n n)$ & $\lambda_{n n}, \mu_{n n n}$ \\
\hline Neutral-different neutral $(n n ')$ & $\lambda_{n n^{\prime}}, \mu_{n n n^{\prime}}, \mu_{n^{\prime} n^{\prime} n}$ \\
\hline
\end{tabular}

The alternative(e.g. Clegg and Brimblecombe, 1990) is

$$
\mu_{n, n, C l^{-}}=0 .
$$

The actual choice is again a database issue. It would be desirable to use conventions for these $\lambda$ and $\mu$ coefficients that are both tied to the same cation or anion, although that is not strictly necessary. Clegg and Brimblecombe (1990) also include coefficients of the form $\mu_{n, n^{\prime}, c}$ and $\mu_{n, n^{\prime}, a}$, where $n$ and $n^{\prime}$ are different neutral species. Here such coefficients will be excluded, as they would require regressing data for aqueous systems more complex than type ternary (see below).

The above set of practical coefficients is fairly classical and should be sufficient for the present toolkit. It is based on the notion that all necessary data should be obtainable by regressing physicochemical data for binary and ternary aqueous systems, all of which are represented by one of the following types: $\mathrm{H} 2 \mathrm{O}-c a, \mathrm{H}_{2} \mathrm{O}-c c^{\prime} a, \mathrm{H}_{2} \mathrm{O}-c a a^{\prime}, \mathrm{H}_{2} \mathrm{O}-n c a, \mathrm{H}_{2} \mathrm{O}-n$, and $\mathrm{H}_{2} \mathrm{O}-n n$ '. Additional types of coefficients can be obtained by examining data for more complex systems. However, this is generally a good point to draw a limit for the Pitzer model based on molality expansion.

Table 3 notes the key parameters other than interaction coefficients. The $A_{\phi}$ parameter is included here, as the exact values of the interaction coefficients may depend on the value assumed in data regression. As noted earlier, the value 0.392 was used in the development of the Harvie et al. (1984) model of the Na-K-Mg-Ca-H-Cl-SO $-\mathrm{OH}-\mathrm{HCO}_{3}-\mathrm{CO}_{3}-\mathrm{CO}_{2}-\mathrm{H}_{2} \mathrm{O}$ system at $25^{\circ} \mathrm{C}$ and in many other works at this particular temperature. $A_{\phi}$ depends on temperature, and most models covering a range of temperature use a temperature function of some kind (see below), and usually that yields a slightly different value at $25^{\circ} \mathrm{C}$. Therefore, this parameter should be treated as a characteristic element of any specific Pitzer model (and corresponding database). A specific model or value for this parameter should not be hard-coded into software. In Pitzer models, the $b$ DebyeHückel parameter is generally treated as a constant value 1.2. The Pitzer $\alpha_{1}, \alpha_{2}$ parameter sets which in part determine the ionic strength dependence of second-order cation-anion interactions 
Table 3: Key Pitzer parameters other than interaction coefficients.

\begin{tabular}{|c|c|}
\hline Parameter & Value(s) \\
\hline$\overline{\overline{A_{\phi}}}$ & $\begin{array}{l}0.392 \text { for the Harvie et al. (1984) model at } \\
25^{\circ} \mathrm{C} \text { and most models at that temperature; } \\
\text { otherwise a function of temperature and pres- } \\
\text { sure. }\end{array}$ \\
\hline$b$ & $\begin{array}{l}\text { Constant value of } 1.2 \text { in all significant known } \\
\text { Pitzer models. }\end{array}$ \\
\hline$\alpha_{1}, \alpha_{2}$ & $\begin{array}{l}\text { 1.2, } 0 \text { for most cation-anion pairs; } \\
1.4,12 \text { for } 2: 2,3: 3 \text {, and } 4: 4 \text { cation-anion pairs } \\
\text { and sometimes other cation-anion pair types. } \\
\text { Other values may be used in certain examples } \\
\text { of Pitzer models. Should be treated in the } \\
\text { present toolkit as potentially unique to each } \\
\text { cation-anion pair. }\end{array}$ \\
\hline
\end{tabular}

(see below) are specific to cation-anion pairs. Here $\alpha_{1}$ is associated with $\beta_{c a}^{1}$ and $\alpha_{2}$ is associated with $\beta_{c a}^{2}$. Originally Pitzer (1973) assigned the values 1.2 and 0 (effectively zero, at least) for most cation-anion pairs, and the value of $\alpha_{2}$ is irrelevant in such instances as $\beta_{c a}^{2}$ is not used (see below). The values 1.4, 12 (and usage of $\beta_{c a}^{2}$ ) were recommended for 2:2 (charge of cation: -charge of anion) pairs, and this was later extended to 3:3, 4:4, etc. Some Pitzer models assign other than these traditional values. The safest procedure is to define the values for each cation-anion pair on a supporting data file and use the traditional values (which are charge dependent) as defaults. This allows maximum flexibility.

The other key Pitzer parameters are interaction parameters of various types. In the development of the theory (Pitzer, 1973), there are second order coefficients (symbolized by $\lambda$ ), and third order coefficients, symbolized by $\mu$.

The corresponding key equations for Pitzer's equations (based on molality expansion) are given below. Here $M$ refers to a cation whose activity coefficient is to be calculated, $X$ to such an anion, and $N$ to such a neutral solute species.

$$
\begin{aligned}
\ln \gamma_{M}= & z_{M}^{2} F+\sum_{a} m_{a}\left[2 B_{M a}+Z C_{M a}\right]+\sum_{c} m_{c}\left(2 \Theta_{M c}+\sum_{a} m_{a} \Psi_{M c a}\right)+ \\
& \sum_{a^{\prime}} \sum_{a<a^{\prime}} m_{a} m_{a^{\prime}} \Psi_{M a a^{\prime}}+\left|z_{M}\right| \sum_{c} \sum_{a} m_{c} m_{a} C_{c a}+ \\
& 2 \sum_{n} m_{n} \lambda_{n M}+\sum_{n} \sum_{a} m_{n} m_{a} \sigma_{n M a}+3 \sum_{n} m_{n}^{2} \mu_{n n M}
\end{aligned}
$$




$$
\begin{aligned}
& \ln \gamma_{X}=z_{X}^{2} F+\sum_{c} m_{c}\left[2 B_{c X}+Z C_{c X}\right]+\sum_{a} m_{a}\left(2 \Theta_{X a}+\sum_{c} m_{c} \Psi_{c X a}\right)+ \\
& \sum_{c^{\prime}} \sum_{c<c^{\prime}} m_{c} m_{c^{\prime}} \Psi_{c c^{\prime} X}+\left|z_{X}\right| \sum_{c} \sum_{a} m_{c} m_{a} C_{c a}+ \\
& 2 \sum_{n} m_{n} \lambda_{n X}+\sum_{n} \sum_{c} m_{n} m_{c} \sigma_{n c X}+3 \sum_{n} m_{n}^{2} \mu_{n n X} ; \\
& \ln \gamma_{N}=2 \sum_{c} m_{c} \lambda_{N c}+2 \sum_{a} m_{a} \lambda_{N a}+\sum_{c} \sum_{a} m_{c} m_{a} \sigma_{N c a}+ \\
& 6 \sum_{c} m_{N} m_{c} \mu_{N N c}+6 \sum_{a} m_{N} m_{a} \mu_{N N a}+ \\
& 2 \sum_{n \neq N} m_{n} \lambda_{N n}+m_{N} \lambda_{N N}+3 \sum_{n} m_{n}^{2} \mu_{N n n}+ \\
& 6 \sum_{n \neq N} m_{N} m_{n} \mu_{N N n} \\
& (\phi-1)\left(\frac{\sum_{i} m_{i}}{2}\right)=\frac{A_{\phi} I^{\frac{3}{2}}}{1+b \sqrt{I}}+\sum_{c} \sum_{a} m_{c} m_{a}\left(B_{c a}^{\phi}+Z C_{c a}\right)+ \\
& \sum_{c^{\prime}} \sum_{c<c^{\prime}} m_{c} m_{c^{\prime}}\left(\Theta_{c c^{\prime}}^{\phi}+\sum_{a} m_{a} \Psi_{c c^{\prime} a}\right)+ \\
& \sum_{a^{\prime}} \sum_{a<a^{\prime}} m_{a} m_{a^{\prime}}\left(\Theta_{a a^{\prime}}^{\phi}+\sum_{c} m_{c} \Psi_{c a a^{\prime}}\right)+ \\
& \sum_{n} \sum_{c} m_{n} m_{c} \lambda_{n c}+\sum_{n} \sum_{a} m_{n} m_{a} \lambda_{n a}+\sum_{n^{\prime}} \sum_{n<n^{\prime}} m_{n} m_{n^{\prime}} \lambda_{n n^{\prime}}+ \\
& \frac{1}{2} \sum_{n} m_{n}^{2} \lambda_{n n}+\sum_{n} m_{n}^{3} \mu_{n n n}+3 \sum_{n^{\prime}} \sum_{n<n^{\prime}} m_{n}^{2} m_{n^{\prime}} \mu_{n n n^{\prime}}+ \\
& 3 \sum_{n} \sum_{c} m_{n}^{2} \mu_{n n c}+3 \sum_{n} \sum_{a} m_{n}^{2} ; \mu_{n n a} \\
& \ln a_{w}=-\left(\frac{\sum_{i} m_{i}}{\Omega}\right) \phi
\end{aligned}
$$

where

$$
\begin{aligned}
F= & -A_{\phi}\left(\frac{\sqrt{I}}{1+b \sqrt{I}}+\frac{2}{b} \ln (1+b \sqrt{I})\right)+\sum_{c} \sum_{a} m_{c} m_{a} B_{c a}^{\prime} \\
& +\sum_{c^{\prime}} \sum_{c<c^{\prime}} m_{c} m_{c^{\prime}} \Theta_{c c^{\prime}}^{\prime}+\sum_{a^{\prime}} \sum_{a<a^{\prime}} m_{a} m_{a^{\prime}} \Theta_{a a^{\prime}}^{\prime}
\end{aligned}
$$




$$
\begin{aligned}
& B_{c a}=\beta_{c a}^{(0)}+\beta_{c a}^{(1)} g\left(\alpha_{1} \sqrt{I}\right)+\beta_{c a}^{(2)} g\left(\alpha_{2} \sqrt{I}\right), \\
& B_{c a}^{\prime}=\beta_{c a}^{(1)} \frac{g\left(\alpha_{1} \sqrt{I}\right)}{I}+\beta_{c a}^{(2)} \frac{g\left(\alpha_{2} \sqrt{I}\right)}{I}, \\
& g(x)=2 \frac{\left(1-(1+x) e^{-x}\right)}{x^{2}}, \\
& g^{\prime}(x)=-2 \frac{\left(1-\left(1+x+\frac{x^{2}}{2}\right) e^{-x}\right)}{x^{2}}, \\
& B_{c a}^{\phi}=B_{c a}+I B_{c a}^{\prime} \quad\left(=\beta_{c a}^{(0)}+\beta_{c a}^{(1)} e^{-\alpha_{1} \sqrt{I}}+\beta_{c a}^{(2)} e^{-\alpha_{2} \sqrt{I}}\right), \\
& Z=\sum_{i} m_{i}\left|z_{i}\right| \\
& C_{M X}=\frac{C_{M X}^{\phi}}{2\left|z_{M} z_{X}\right|^{\frac{1}{2}}} \\
& \Theta_{c c^{\prime}}={ }^{S} \Theta_{c c^{\prime}}+{ }^{E} \Theta_{c c^{\prime}} \\
& \Theta_{a a^{\prime}}={ }^{S} \Theta_{a a^{\prime}}+{ }^{E} \Theta_{a a^{\prime}}, \\
& \Theta_{c c^{\prime}}^{\prime}={ }^{E} \Theta_{c c^{\prime}}^{\prime}, \\
& \Theta_{a a^{\prime}}^{\prime}={ }^{E} \Theta_{a a^{\prime}}^{\prime}, \\
& { }^{E} \Theta_{c c^{\prime}}=\left(\frac{z_{c} z_{c^{\prime}}}{4 I}\right)\left(J\left(x_{c c^{\prime}}\right)-\frac{J\left(x_{c c}\right)}{2}-\frac{J\left(x_{c^{\prime} c^{\prime}}\right)}{2}\right), \\
& { }^{E} \Theta_{a a^{\prime}}=\left(\frac{z_{a} z_{a^{\prime}}}{4 I}\right)\left(J\left(x_{a a^{\prime}}\right)-\frac{J\left(x_{a a}\right)}{2}-\frac{J\left(x_{a^{\prime} a^{\prime}}\right)}{2}\right), \\
& { }^{E} \Theta_{c c^{\prime}}^{\prime}=-\frac{{ }^{E} \Theta_{c c^{\prime}}}{I}+\left(\frac{z_{c} z_{c^{\prime}}}{8 I^{2}}\right)\left(x_{c c^{\prime}} J^{\prime}\left(x_{c c^{\prime}}\right)-\frac{x_{c c} J^{\prime}\left(x_{c c}\right)}{2}-\frac{x_{c^{\prime} c^{\prime}} J^{\prime}\left(x_{c^{\prime} c^{\prime}}\right)}{2}\right), \\
& { }^{E} \Theta_{a a^{\prime}}^{\prime}=-\frac{{ }^{E} \Theta_{a a^{\prime}}}{I}+\left(\frac{z_{a} z_{a^{\prime}}}{8 I^{2}}\right)\left(x_{a a^{\prime}} J^{\prime}\left(x_{a a^{\prime}}\right)-\frac{x_{a a} J^{\prime}\left(x_{a a}\right)}{2}-\frac{x_{a^{\prime} a^{\prime}} J^{\prime}\left(x_{a^{\prime} a^{\prime}}\right)}{2}\right), \\
& x_{i j}=6 z_{i} z_{j} A^{\phi} \sqrt{I} \quad\left(i j \text { can be } c c^{\prime}, c c, c^{\prime} c^{\prime}, a a^{\prime}, a a, \text { or } a^{\prime} a^{\prime}\right) \text {, } \\
& \Theta_{c c^{\prime}}^{\phi}={ }^{S} \Theta_{c c^{\prime}}+{ }^{E} \Theta_{c c^{\prime}}+I^{E} \Theta_{c c^{\prime}}^{\prime}, \\
& \Theta_{a a^{\prime}}^{\phi}={ }^{S} \Theta_{a a^{\prime}}+{ }^{E} \Theta_{a a^{\prime}}+I^{E} \Theta_{a a^{\prime}}^{\prime} .
\end{aligned}
$$

Harvie (1981) proposed a method of using two Chebyshev polynomial approximations to calculate $J(x)$ and $J^{\prime}(x)$. One polynomial is used for Region I $(x=1)$ and the other for Region II $(x>1)$. Table 4 contains the requisite coefficients. The equations are as follows: 
Region I $(x=1)$ :

$$
\begin{aligned}
z & =x^{5}-2 \\
\frac{d z}{d x} & =\frac{4}{5} x^{-\frac{4}{5}} \\
b_{k} & =z b_{k+1}-b_{k+2}+a_{k}^{I}, \quad k=0, \ldots, 20 \\
d_{k} & =b_{k+1}+z d_{k+1}-d_{k+2}, \quad k=0, \ldots, 20
\end{aligned}
$$

Region II $(x>1)$ :

$$
\begin{aligned}
z & =\frac{40}{9} x^{-\frac{1}{10}}-\frac{22}{9}, \\
\frac{d z}{d x} & =-\frac{40}{90} x^{-\frac{11}{10}} \\
b_{k} & =z b_{k+1}-b_{k+2}+a_{k}^{I I}, \quad k=0,20, \\
d_{k} & =b_{k+1}+z d_{k+1}-d_{k+2}, \quad k=0,20 .
\end{aligned}
$$

The final equations are

$$
\begin{aligned}
J(x) & =\frac{1}{4} x-1+\frac{1}{2}\left(b_{0}-b_{2}\right) \\
J^{\prime}(x) & =\frac{1}{4}+\frac{1}{2} \frac{d z}{d x}\left(d_{0}-d_{2}\right) .
\end{aligned}
$$

When temperature dependence is considered, the various Pitzer parameters are generally described by empirical temperature functions. The recommended temperature function for the present toolkit is

$$
A=a_{1}+a_{2}\left(\frac{1}{T}-\frac{1}{T_{r}}\right)+a_{3} \ln \left(\frac{T}{T_{r}}\right)+a_{4}\left(T-T_{r}\right)+a_{5}\left(T^{2}-T_{r}^{2}\right) .
$$

where $A$ is the parameter to be calculated, $a_{1}-a_{5}$ are the associated constants, $T$ is the absolute temperature, and $T_{r}$ is the reference temperature $(298.15 \mathrm{~K})$. More elaborate forms are not recommended. Also, it is preferred to use the term in temperature-squared only if the precedinig four terms are inadequate. More recent work is likely to be consistent with the above form in the first four terms, although the terms might be in a different order. It is noted that the eight-term form proposed by Greenber and Moller (1989) is overly complex and contains singularities with respect to temperature. When data are encountered in such a form, they should be recast into the more reasonable form given above. This has been done for example for the Greenber and Moller (1989) data in the Yucca Mountain Project Pitzer thermodynamic database (see Sandia National Laboratories, 2007, Appendix I). 
Process Model Mathematical Formulation Requirements

Table 4: Chebyshev polynomial coefficients for computing $J(x)$ and $J^{\prime}(x)$ (Harvie, 1981)

\begin{tabular}{rrr}
$k$ & $a_{k}^{I}$ & $a_{k}^{I I}$ \\
\hline 0 & 1.925154014814667 & 0.628023320520852 \\
\hline 1 & -0.060076477753119 & 0.462762985338493 \\
\hline 2 & -0.029779077456514 & 0.150044637187895 \\
\hline 3 & -0.007299499690937 & -0.028796057604906 \\
\hline 4 & 0.000388260636404 & -0.036552745910311 \\
\hline 5 & 0.000636874599598 & -0.001668087945272 \\
\hline 6 & 0.000036583601823 & 0.006519840398744 \\
\hline 7 & -0.000045036975204 & 0.001130378079086 \\
\hline 8 & -0.000004537895710 & -0.000887171310131 \\
\hline 9 & 0.000002937706971 & -0.000242107641309 \\
\hline 10 & 0.000000396566462 & 0.000087294451594 \\
\hline 11 & -0.000000202099617 & 0.000034682122751 \\
\hline 12 & -0.000000025267769 & -0.000004583768938 \\
\hline 13 & 0.000000013522610 & -0.000003548684306 \\
\hline 14 & 0.000000001229405 & -0.000000250453880 \\
\hline 15 & -0.000000000821969 & 0.000000216991779 \\
\hline 16 & -0.000000000050847 & 0.000000080779570 \\
\hline 17 & 0.000000000046333 & 0.000000004558555 \\
\hline 18 & 0.000000000001943 & -0.000000006944757 \\
\hline 19 & -0.000000000002563 & -0.000000002849257 \\
\hline 20 & -0.000000000010991 & 0.000000000237816 \\
\hline
\end{tabular}




\subsubsection{Extended UNIQUAC}

The Extended UNIQUAC model is another high ionic strength model. It differs from the Pitzer model in various ways, but the most striking is the elimination of triplet interaction parameters and the appearance of a small number of "singlet" parameters in addition to the charge number. These differences result in a striking reduction in the number of parameters required to model a system containing a large number of solute species. Extended UNIQUAC is fundamentally based on mole fractions. Molal activity coefficients for the solute species are therefore derived the mole fraction counterparts. Also, water (the solvent) is considered in the relevant pair interactions.

There are presently multiple "flavors" of Extended UNIQUAC. The one that will be addressed here is based on Thomsen $(1997,2005)$ and various other papers, of which Thomsen is usually a co-author. The reason for this choice is that this particular flavor has been openly developed to cover a wide range of useful components, while others are less extensively developed or are at least in significant part proprietary. The original model covers the system containing the solute species $\mathrm{Na}^{+}, \mathrm{K}^{+}, \mathrm{NH}_{4}^{+}, \mathrm{H}^{+}, \mathrm{Cl}^{-}, \mathrm{NO}_{3}^{-}, \mathrm{SO}_{4}^{2-}, \mathrm{HSO}_{4}^{-}, \mathrm{OH}^{-}, \mathrm{CO}_{3}^{2-}, \mathrm{HCO}_{3}^{-}$, and $\mathrm{S}_{2} \mathrm{O}_{8}^{2-}$ from 25 to about $110^{\circ} \mathrm{C}$. The number of chemical species treated has been significantly extended in subsequent development. Thomsen (2005) provides the preferred overview of the model, while Thomsen (1997) provides more detail. This model has been incorporated into an as yet unreleased version of EQ3/6 (and a corresponding supporting thermodynamic data file exists).

The effectiveness of the Extended UNIQUAC model is comparable to the molality-based Pitzer model described above. It may have an advantage at smaller mole fractions of water, as it this is a mole fraction-based model. It is notably weaker at elevated temperature, due to the simple temperature dependence currently applied to the pair interaction parameters, as noted below. That weakness could be easily remedied, however. The Extended UNIQUAC model is becoming increasing popular since, compared with the Pitzer's model (in either molality- or mole fractionbased form), it produces comparably good results with fewer parameters that need to be fitted to experimental data.

The model utilizes the following singlet parameters: $r_{i}$ and $q_{i}$ ("volume" and "surface area" parameters, respectively). These are treated as constant in regard to temperature. The model also uses the $u_{k i}$ pair interaction parameter, which is treated as a function of temperature:

$$
u_{k i}=u_{k i}^{0}+u_{k i}^{t}\left(T-T_{r}\right)
$$

where $T$ is the absolute temperature and $T_{r}$ is the reference temperature $(298.15 \mathrm{~K})$. Here both $k$ and $i$ span the range of component species, including solvent water. The combination in which $k$ and $i$ are equal is included. For example, the pair combination $\mathrm{H}_{2} \mathrm{O}-\mathrm{H}_{2} \mathrm{O}$ is included. The $u$ coefficients are symmetrical $\left(u_{k l}=u_{l k}\right)$. The linear representation of temperature dependence is probably a limiting factor in regard to the temperature range of the model. A three-parameter form would likely be required to significantly extend the temperature range.

The $r_{i}$ and $q_{i}$ are used to calculate the following quantities:

$$
\begin{gathered}
\phi_{i}=\frac{x_{i} r_{i}}{\sum_{l} x_{l} r_{l}}, \quad(\text { the "volume fraction"); } \\
\theta_{i}=\frac{x_{i} q_{i}}{\sum_{l} x_{l} q_{l}}, \quad \text { (the "surface area fraction"). }
\end{gathered}
$$


In the above equations, $l$ spans all component species (including solvent water). Here $x$ denotes mole fraction. Note that for a solute species, the mole fraction is given by

$$
x_{i}=\frac{m_{i}}{\Omega+\sum_{j} m_{j}}
$$

where $j$ spans only the solute species. For solvent water $(w)$, the mole fraction is given by

$$
x_{w}=\frac{\Omega}{\Omega+\sum_{j} m_{j}} .
$$

The $u_{k i}$ pair interaction parameter is used to calculate

$$
\psi_{k i}=\exp \left(-\frac{u_{k i}-u_{i i}}{T}\right)
$$

Note that symmetry does not apply to this parameter $\left(\psi_{i k}\right.$ does not necessarily equal $\psi_{k i}$, because $u_{i i}$ does not necessarily equal $u_{k k}$ ).

The Extended UNIQUAC theory first addresses mole fraction (or "rational") activity coefficients, symbolized here by $f$ (by $\lambda$ in earlier discussion). These are calculated by first obtaining characteristic parts. In UNIQUAC theory, these include the "combinatorial" $(C)$ or entropic part and "residual" $(R)$ or enthalpic part. For the mole fraction activity coefficient these parts are respectively given by

$$
\begin{aligned}
& \ln f_{i}^{C}=\ln \left(\frac{\phi_{i}}{x_{i}}\right)+1-\frac{\phi_{i}}{x_{i}}-\frac{z}{2} q_{i}\left[\ln \left(\frac{\phi_{i}}{\theta_{i}}\right)+1-\frac{\phi_{i}}{\theta_{i}}\right], \\
& \ln f_{i}^{R}=q_{i}\left[1-\ln \left(\sum_{k} \theta_{k} \psi_{k i}\right)-\sum_{k} \frac{\theta_{k} \psi_{i k}}{\sum_{l} \theta_{l} \psi_{l k}}\right],
\end{aligned}
$$

where $i$ spans all component species. Here $z$ in the combinatorial part is the coordination number, assigned a value of 10 .

The corresponding infinite dilution terms are obtained by setting $x_{w}=1$ :

$$
\begin{aligned}
\ln f_{i}^{\infty, C} & =\ln \left(\frac{r_{i}}{r_{w}}\right)+1-\frac{r_{i}}{r_{w}}-\frac{z}{2} q_{i}\left[\ln \left(\frac{r_{i} q_{w}}{r_{w} q_{i}}\right)+1-\frac{r_{i} q_{w}}{r_{w} q_{i}}\right], \\
\ln f_{i}^{\infty, R} & =q_{i}\left[1-\ln \psi_{w i}-\psi_{w i}\right] .
\end{aligned}
$$

These infinite dilution terms are only pertinent to the solute species. They are required to obtain the mole fraction activity coefficients for solute species for an infinite dilution reference $\left(f_{i}=1\right.$ at infinite dilution, instead of at $x_{i}=1$ ).

The desired mole fraction activity coefficients are obtained by combining the combinatorial and residual parts with a Debye-Hückel part, which is based on the Debye-Hückel-charging model .

The activity coefficient of water is then given by

$$
\ln f_{w}=\ln f_{w}^{C}+\ln f_{w}^{R}+\frac{1}{\Omega}\left(\frac{2 A_{\gamma, e}}{b^{3}} \sigma(b \sqrt{I)})\right.
$$


where $b$ is assigned the familiar value of 1.5 . Since $f_{w}$ is the same as $\lambda_{w}$, the activity of water can be obtained from

$$
\ln a_{w}=\ln x_{w}+\ln f_{w} .
$$

The osmotic coefficient, if desired, can be obtained from the activity of water using the relation given in previous sections.

The mole fraction activity coefficient of a solute species is given by

$$
\ln f_{i}=-\frac{A_{\gamma, e} z_{i}^{2} \sqrt{I}}{1+b \sqrt{I}}+\ln f_{i}^{C}-\ln f_{i}^{\infty, C}+\ln f_{i}^{R}-\ln f_{i}^{\infty, R} .
$$

Here $A_{\gamma, e}=\ln (10) A_{\gamma, 10}$. The molal activity coefficient of a solute species can then be obtained using

$$
\ln \gamma_{i}=\ln f_{i}+\ln x_{w}
$$




\subsubsection{NEA SIT}

The NEA SIT model is a "Specfic Interactions Theory" model adopted by the European Nuclear Energy Agency (NEA) for use in its international program to develop thermodynamic data sets for mostly radionuclide elements of concern to geologic disposal of high level nuclear waste. The term specific interactions theory is generically used to describe theories in which the activity coefficients pertinent to aqueous solutions have a general dependence on specific solution composition. In particular, it is recognized that the ionic strength is insufficient to fully describe this dependence. Recall that Pitzer's equations also represent a "specific interaction theory." Therefore, the present model, often referred to as just "SIT," will here be referred to as "NEA SIT" to avoid potential confusion.

The NEA SIT model was basically adopted to provide activity coefficient corrections to measured data, so as to yield true equilibrium constants (mostly complexation constants). The model in the modern context traces to Grenthe et al. (1992), who used it in a study of the thermodynamic properties of aqueous, solid, and gas uranium-bearing species. The model was described in their Appendix B, "Ionic strength corrections". The model has been more recently discussed by Grenthe et al. (1997), whose title more appropriately includes "medium effects." They also introduced some minor modification in regard to the treatment of neutral aqueous species.

The NEA SIT model has been further developed in other volumes in the NEA "Chemical Thermodynamics" series (the uranium volume was the first in the series). Although there has always been a strong focus on species pertinent to radionuclide species, it was recognized from the start that analyzing data pertinent to aqueous media would require extending the model to include necessary species that would be part of the media. These "auxiliary data" include extension of the NEA SIT model to handle pertinent non-radionuclide species.

Although there has been great interest in and usage of the standard state thermodynamic data produced by the NEA project, there has been less implementation of the NEA SIT activity coefficient model in pertinent modeling codes and applications. There has thus far been little testing of the ability of the NEA SIT model to handle systems of geochemical interest, which would provide a stronger test of the adequacy of the model, in particular regard to the adequacy of the auxiliary data. This situation may be changing, as it has recently been announced that NEA SIT has been added as an option to the modeling code PHREEQC.

The origins of the NEA SIT theory basically go back to Guggenheim (1935), although the present model includes some modifications, mostly tracing to Ciavatta (1980). A helpful way to look at the model is to compare it with Pitzer's equations. Basically, the equations are like Pitzer's equations, except that third-order interactions are excluded. NEA SIT uses a form of the DebyeHückel-charging model instead of Pitzer's Debye-Hückel-osmotic model for the Debye-Hückelterm. The second order interactions are treated as a function of ionic strength, somewhat similar to the treatment in Pitzer's equations. This treatment distinguishes the NEA SIT model from the Guggenheim (1935) model, which did not include such ionic strength dependence.

The relevant equations are

$$
\log _{10} \gamma_{j}=\frac{-z_{j}^{2} A_{\gamma, 10} \sqrt{I}}{1+1.5 \sqrt{I}}+\sum_{k} \varepsilon_{j k} m_{k}
$$


where $\varepsilon_{j k}$ is a second-order interaction coefficient. This is treated in the general case as a function of ionic strength as previously recommended by Ciavatta (1980):

$$
\varepsilon_{j k}=\varepsilon_{1, j k}+\varepsilon_{2, j k} \log _{10} I .
$$

In many instances, however, it may simply be treated as a constant (e.g., $\varepsilon_{j k}=\varepsilon_{1, j k}$ ).

It is assumed (Grenthe et al., 1992, p. 686, Assumption 2) that there are no pair-wise interactions among ions of the same charge sign and for uncharged species. More simply put, the only nonzero interactions are for cation-anion combinations. Grenthe et al. (1997) removed the restriction regarding uncharged species, but appear to have considered only ion-neutral interactions.

There are difficulties with the Ciavatta (1980) representation of the ionic strength dependence of the second-order interaction coefficient. The magnitude of the term in $\log 10 \mathrm{I}$ approaches infinity as $I$ approaches zero. Note that

$$
\varepsilon_{j k}^{\prime}=\frac{\partial \varepsilon_{j k}}{\partial I}=\frac{\varepsilon_{2, j k}}{\ln (10) I} .
$$

The derivative becomes singular as $I$ approaches zero Also, although $\varepsilon_{j k}$ at first seems to be the analogue of the Pitzer $\lambda_{i j}$ (differing by a factor of two, with $j k$ substituting for $i j$ ), it really isn't. This can be seen by comparing the NEA SIT equation for the solute activity coefficient with that for Pitzer's equations. The NEA SIT equation lacks a term containing $\varepsilon_{j k}^{\prime}$ analogous to the term in $\lambda_{i j}^{\prime}$ appearing in the Pitzer equation (and which is second order in molality). One should therefore expect that the NEA SIT equation 1) may not satisfy the thermodynamic consistency relations, and 2) may be at best only quasi-consistent with a corresponding equation for the activity of water. Grenthe et al. (1997) reviewed similarities and differences between the NEA SIT equations and Pitzer's equations but unfortunately did not address the consistency issue in the former. This issue will be addressed further later in this section.

The omission of treatment of third-order interactions and the restriction of the treatment of secondorder interactions to cation-anion cases only provides for a much simplified model. However, it is likely to also restrict the range of accuracy (as may the suspected consistency problem noted above). Indeed, the model is only intended for application in a moderate range of ionic strength. Grenthe et al. (1992, p. 685) note that the use of the 1.5 value in the denominator of the DebyeHückel term was found by Ciavatta (1980) to provide useful fits in the ionic strength range $0.5-3.5 \mathrm{~m}$ and note its correlation to regressed values of second-order interaction coefficients. The actual application of the NEA SIT model in the NEA "Chemical Thermodynamics" series is generally for maximum ionic strengths in the 1-2 molal range. In the absence of additional testing, an upper limit of ionic strength for using this model would appear to be something like 2-4 molal. This is sufficient to be useful for many applications, but clearly falls short of what can be done using a model based on Pitzer's equations or Extended UNIQUAC.

Grenthe et al. (1992, Table B.1, p. 685) recommend using the values of Helgeson et al. (1981) for the $A_{\gamma, 10}$ parameter, which they simply refer to as $A$. They note its temperature dependence, but do not recommend a specific means of describing this.

Grenthe et al. (1992) do not present a general equation for the activity of water. Their primary recommendation is to calculate the activity of water from the osmotic coefficient, using data tables for pure aqueous electrolytes and electrolyte mixtures that correspond to the relevant electrolyte media. They do suggest (p. 688, equation B.12) a formulation that is specific to a pure electrolyte 
solution. This does not specifically address the consistency problem that may arise if the interaction coefficient is dependent on ionic strength, and the derivative of this coefficient is ignored.

If one tests the activity coefficient equation for self-consistency using the relation

$$
\frac{\partial \ln \gamma_{j}}{\partial m_{i}}=\frac{\partial \ln \gamma_{i}}{\partial m_{j}}
$$

one obtains, after making appropriate substitutions and simplification, that the following residual would have to be satisified:

$$
\left(\sum_{k} m_{k} \varepsilon_{2, j k}\right) \frac{z_{i}^{2}}{2}=\left(\sum_{k} m_{k^{\prime}} \varepsilon_{2, i k}\right) \frac{z_{j}^{2}}{2} .
$$

This is not likely to be the case unless ions $i$ and $j$ have the same magnitude of charge number and the two summations happen to be equal. The summations are likely to be equal only under restricted circumstances. For example, if $i$ and $j$ are both cations and $k$ spans the anions (ignoring neutral species), then $\varepsilon_{2}$, would have to have the same value for all cation-anion pairs. Other assumptions about $i$ and $j$ lead to the same result (or similar results if neutral species are considered). This problem vanishes if the total interaction coefficients are treated as constants (e.g., $\left.\varepsilon_{j k}=\varepsilon_{1, i k}, \varepsilon_{2, i k}=0\right)$.

The following approximation for the activity of water is recommended for the present toolkit when using the NEA SIT model:

$$
\log a_{w}=\frac{1}{\Omega}\left(-\frac{\sum_{i} m_{i}}{\ln (10)}+\frac{2}{3} A_{\gamma, 10} I^{\frac{3}{2}} \sigma(1.5 \sqrt{I})-\sum_{j k} \varepsilon_{j k} m_{j} m_{k}\right) .
$$

This is only a quasi-consistent form due to the points noted above.

The NEA SIT model does not seem to have a specific recommended form for the temperature dependence of the coefficients $\varepsilon_{1}$ and $\varepsilon_{2}$. This is apparently because the model is mainly developed for $25^{\circ} \mathrm{C}$. Grenthe et al. (1992) largely avoid the issue. Grenthe et al. (1997) give a detailed discussion of thermodynamic properties related to elevated temperature and pressure. However, they give no generally useful form for a practical treatment of the temperature dependence. To use their equations, one would need tabulated values of various derivative properties, which are only available for a relatively small number of species. Therefore, in practice, this model is restricted to $25^{\circ} \mathrm{C}$ until someone does further development. 


\subsubsection{Rescaling Ionic Activity Coefficients}

The activity coefficient models described above include descriptions of individual ion activity coefficients. This is problematic in that ionic activity coefficients and ionic activities are not measurable for individual ions. These quantities are measurable only in combinations that correspond to electrical neutrality. For activity coefficients, examples of such combinations include $\log \gamma_{H^{+}}+\log \gamma_{C l^{-}}$and $2 \log \gamma_{H^{+}}+\log \gamma_{S O_{4}^{2-}}$; examples for activities are analogous. Molalities of individual ions are measureable (or quantifiable by inference). Thus, if one could obtain or specify the activity or activity coefficient of one single ion in an aqueous solution, one could then use this as a reference to obtain the activities and activity coefficients of all other ions present in the same solution.

The need to define activity coefficients and activities for individual ionic species is dealt with by the use of a "splitting" convention. Such a convention is at least somewhat arbitrary, although it may be guided in part by theoretical concerns. One could address the issue by adopting the results of model equations for single ion activity coefficients. The model equations for these are all in part arbitrary, implicitly including a splitting based on some combination of theoretical notions and pleasing (but not necessarily unique) symmetry. The problem with just using the model equations in their native form is that other conventions have been previously adopted into measurement practice, particularly the measurement of $\mathrm{pH}$. For accurate modeling consistent with standard analytical chemistry practice, it becomes necessary to rescale the results of the model equations presented above. This only affects the activity coefficients of ionic species. For most analytical splitting conventions, some expression is specified for the activity coefficient of a reference ion, usually $\mathrm{Cl}^{-}$or $\mathrm{H}^{+}$.

The most significant analytical splitting conventions for aqueous ions are tied to the definition of the $\mathrm{pH}$. Conceptually,

$$
p H=-\log a_{H^{+}} .
$$

In order to provide a practical basis for measuring the $\mathrm{pH}$, it is necessary to define the activity of the hydrogen ion. The splitting convention used for this purpose then defines a $\mathrm{pH}$ scale. The choice of $\mathrm{pH}$ scale further affects the definition of the redox potential, $\mathrm{E}_{h}$.

In modern work, the dominant $\mathrm{pH}$ scale is the NBS scale, originated by the National Bureau of Standards, now the National Institute of Standards and Technology. The NBS scale is based on the Bates-Guggenhiem equation (Bates, 1964):

$$
\log \gamma_{C l^{-}}=-\frac{A_{\gamma, 10} \sqrt{\bar{I}}}{1+1.5 \sqrt{\bar{I}}} .
$$

This is a simple form of the extended Debye-Hückel equation. It defines the activity coefficient of the chloride ion. It may be surprising that chloride is used as the reference ion rather than the hydrogen ion, which is more closely tied to the $\mathrm{pH}$. What is apparent is that the Bates-Guggenheim equation must give a result that is different from what would be obtained for the chloride ion using say the Davies equation or the B-dot equation, or for that matter, from Pitzer's equations. In the range of low ionic strength (say less than 1 molal), the differences should be numerically small for each of the three practical models, as they and the Bates-Guggenheim equation all include some form of extended Debye-Hückel model and thus are all consistent with the Debye-Hückel limiting 
law. At higher ionic strength, however, the differences can be substantial (the equivalent of several $\mathrm{pH}$ units).

The Bates-Guggenheim equation can be applied whether or not there is any chloride in aqueous solution, as the equation is sufficient to calculate the specified activity coefficient. The charge number of -1 is effectively built into the equation.

The Bates-Guggenheim equation (the NBS pH scale) is effectively built in to the calibration of all modern means of measuring the $\mathrm{pH}$, whether in $\mathrm{pH}$ calibration buffers or $\mathrm{pH}$ paper. EQ3/6 for example by default rescales ionic activity coefficients computed from the models to be consistent with the NBS pH scale (other options, including no rescaling, may be offered). Rescaling from one scale (scale "1") to another (scale " 2 ") is accomplished using

$$
\log \gamma_{i}^{(2)}=\log \gamma_{i}^{(1)}+\frac{z_{i}}{z_{j}}\left(\log \gamma_{j}^{(2)}-\log \gamma_{j}^{(1)}\right)
$$

Here $j$ denotes the reference ion (here $\mathrm{Cl}^{-}$) and $i$ denotes any ion (including the reference ion). In the present context, scale " 1 " is usually that implied by a model equation and scale " 2 " is the desired scale.

An alternative convention is to choose

$$
\log \gamma_{H^{+}}=0
$$

For the hydrogen ion, this results in

$$
p H=-\log m_{H^{+}} .
$$

as the activity and molality of the hydrogen ion are numerically equivalent. The rescaling of ionic activity coefficients for consistency does not give an analogous result for other ions. EQ3/6 allows rescaling using this convention as an option, but it has limited utility and it not recommended as a general option in the present toolset.

The definition of the $\mathrm{pH}$ in terms of molality ("pmH") is significant independent of rescaling. Thus one has simply

$$
p m H=-\log m_{H^{+}} .
$$

In concentrated electrolyte solutions (e.g., WIPP, Hanford tanks), pmH is often more useful for assessing the acidity/basicity of a solution than the NBS pH. The NBS pH cannot be accurately measured in concentrated solutions owing to liquid junction effects with electrodes and interferences with dyes. Also, the Bates-Guggenheim equation (and the NBS pH scale itself) was originally intended for use only at low ionic strength. Bates (1964) suggested application to solutions with ionic strengths of no greater than 0.1 molal. Since then, however, the scale has been used at higher ionic strengths. This has led to the problem that of two highly concentrated solutions with an NBS pH of say 2, one might be acidic (in the sense that $\mathrm{H}+$ is abundant) and the other not (in the sense that $\mathrm{H}+$ is not abundant). In other words, the common association of $\mathrm{pH}$ values with various degrees of acidity/basicity (e.g., 7 is neutral) no longer applies.

Still other conventions and scales exist. However, for the present toolset only the following is required. First, the default behavior will be to apply rescaling to the NBS scale. Second, the option 
will be available to use the basic model results without rescaling. Third, the pmH will be directly calculated and included in the output. An option to rescale the activity coefficients for consistency with the $\log \gamma_{H^{+}}=0$ convention will not be required.

\subsection{Sorption}

\subsubsection{Overview}

Sorption involves the attachment of dissolved and/or colloidal species to mineral or other solid surfaces. Sorption has the effect of slowing the effective transport rate of a species through porous media through its retardation effect. The retardation effect for a species, $R_{f}$, is given by (Bouwer, 1991)

$$
R_{f}=\frac{V_{g w}}{V_{s p}}
$$

where $V_{g w}$ is the velocity of the groundwater and $V_{s p}$ is the velocity of the species. A variety of models have been used to describe sorption and can be broadly divided into those that describe it as a bulk process versus those that are mineral or solid phase specific. The latter approach involves the calculation of bulk sorption from the sum of sorption on individual solid phases, an assumption referred to as Component Additivity. Within the class of bulk sorption models, a distinction can be made between those which assume a finite number of sorption sites (these are referred to as showing Langmuir type behavior and include the Langmuir isotherm itself and most surface complexation and ion exchange models) and those that assume either an infinite sorption capacity or at least a capacity that is not tightly constrained (these include the linear distribution coefficient and the nonlinear Freundlich isotherm). Alternatively, one could also distinguish between single component, non-competitive models (e.g., Langmuir and Freundlich) and multicomponent competitive models (surface complexation and ion exchange).

Another possible distinction is between equilibrium and kinetic sorption models. In many cases, the formulations for the equilibrium and kinetic cases differ only insofar as the kinetic case involves involves a thermodynamic driving force (as in the equilibrium case), but modified by a finite rate constant. In some cases, however, sorption is described as irreversible, which implies that there is no back reaction (desorption).

\subsubsection{Process Model Equations}

Linear Distribution Coefficients $\left(K_{d}\right)$. A simple approach to describe metal or ionic radionuclide sorption by a sediment,

$$
A_{a q} \leftrightharpoons A_{a d s}
$$

is to use a constant distribution coefficient, defined by:

$$
K_{d}=\frac{\left[A_{a d s}\right]}{\left[A_{a q}\right]},
$$

where $K_{d}$ is the distribution coefficient $(\mathrm{L} / \mathrm{kg}),\left[A_{a d s}\right]$ is the sorbed concentration $(\mathrm{mol} / \mathrm{kg})$ to the bulk solid phase, and $\left[A_{a q}\right]$ is the total dissolved concentration in groundwater ( $\mathrm{mol} / \mathrm{L}$ ) (Davis and 
Kent, 1990). One of the key advantages of representing sorption with a distribution coefficient is that it can be easily incorporated into reactive transport models used for migration predictions.

Equation (5.89) shows that if one assumes that the amount of sorption is proportional to the dissolved concentration, then there is a linear relationship where the $K_{d}$ value is the slope. In this simple case, referred to as a linear isotherm, retardation of a concentration front in simple porous media is given by

$$
\frac{\bar{v}}{\bar{v}_{c}}=1+\frac{\rho_{b}}{n} K_{d}
$$

where $\rho_{b}$ is the bulk density, $n$ the porosity, $\bar{v}$ the average linear velocity of the groundwater and $\bar{v}_{c}$ the velocity of the point on the concentration profile where the concentration is half that of the input concentration (Freeze and Cherry, 1979). Note that the ratio $\bar{v} / \bar{v}_{c}$ here is the retardation factor and represents the retardation of the movement of front relative to the flowing groundwater. While this is a simplified example, it serves to illustrate the key point that the $K_{d}$ value directly influences predictions of adsorbing metal or radionuclide mobility.

Assumptions and Applicability Sorption is proportional to the dissolved concentration. The aqueous and adsorbed phases are in equilibrium.

Data Needs Typically $K_{d}$ values are determined for a particular subsurface material from the slope of a fitted line to the concentration of the sorbed species, $A_{a d s}$, plotted versus the dissolved concentration of the same species, $A_{a q}$. These data may be derived from laboratory analyses, where one typically varies the dissolved concentrations systematically, or they may be derived from in situ field data. Since $K_{d}$ values may be variable, and in particular a function of temperature, $\mathrm{pH}$ or the redox state of the system (see below), it is often necessary to compile them in a lookup table for use by a particular computer code.

Langmuir Isotherm. The Langmuir isotherm assumes that the sorption sites, S, on the surface of a solid (absorbent) become occupied by an absorbate from the solution, A. Implying a 1:1 stoichiometry

$$
S+A \leftrightharpoons S A
$$

where $S A$ is the adsorbed species on the surface. At equilibrium, a standard mass action equation can be written:

$$
K_{a d s, L}=\frac{[S A]}{[S]\{A\}},
$$

where the square brackets here refer to the concentration of the species or site, and the curly brackets refer to the aqueous activity. Using the maximum concentration of surface sites, $S_{T}$

$$
\left[S_{T}\right]=[S]+[S A]
$$


one can write the Langmuir isotherm in its familiar hyperbolic form

$$
[S A]=\left[S_{T}\right] \frac{K_{a d s}\{A\}}{1+K_{a d s}\{A\}} .
$$

Assumptions For the following, it is assumed that the surface and aqueous species are in equilibrium.

Data Needs The equilbrium constant, $K_{a d s}$, is typically obtained from experimental data. It depends on the specified absorbent and absorbate, and may be a function of temperature. It may be calculated from:

$$
K_{a d s}=\exp \left(\frac{-\Delta G_{a d s}^{\circ}}{R T}\right),
$$

where $\Delta G$ is the change in free energy for the reaction, typically obtained from a database, $R$ is the gas constant and $T$ is the temperature.

Freundlich. The Freundlich isotherm is another equilibrium model for sorption of absorabte A onto sorption sites, $\mathrm{S}$.

$$
S+A \leftrightharpoons S A
$$

Represented by the mass action equation:

$$
K_{a d s, F}=\frac{[S A]}{\{A\}^{\beta_{F}}},
$$

where the square brackets again refer to the concentration of the species or site, the curly brackets refer to the aqueous activity. $K_{a d s, F}$ and $\beta_{F}$ are the Freundlich parameters (e.g. Langmuir, 1997; Stumm, 1992).

Assumptions For the following, it is assumed that the surface and aqueous species are in equilibrium.

Data Needs The Freundlich parameters, $K_{a d s, F}$ and $n$, are generally obtained by fits to experimental data for a specific surface and aqueous species. They will generally be obtained from a database, and may be represented by a functional form or lookup table.

Multi-site, Multi-component Ion Exchange. An ion exchange reaction can be described via a mass action expression with an associated equilibrium constant (Appelo and Postma, 1993; Sposito and Sparks, 1981; Vanselow, 1932). The exchange reaction can be written in generic form as

$$
v A C l_{u}(a q)+u B X_{\nu}(s) \leftrightharpoons u B C l_{\nu}(a q)+v A X_{u}(s)
$$


where $\mathrm{X}$ refers to the exchange site occupied by the cations $A^{u+}$ and $B^{v+}$. The equilibrium constant, $K_{e q}$, for this reaction can be written as (Vanselow, 1932)

$$
K_{e q}=\frac{\left\{B C l_{\nu}\right\}^{u}\left\{A X_{u}\right\}^{\nu}}{\left\{A C l_{u}\right\}^{\nu}\left\{B X_{\nu}\right\}^{u}}
$$

where the curly braces refer to the thermodynamic activities. Several activity conventions are in wide use. One possibility is the Gaines-Thomas activity convention, which assumes a reaction stoichiometry of the following form (Appelo and Postma, 1993), written here assuming the $\mathrm{Cs}^{+}$is the relevant cation of interest

$$
C s^{+}+(1 / z) M X(i)_{z} \leftrightharpoons C s X(i)+(1 / z) M^{z+}
$$

where $M$ is the competing cation $\left(\mathrm{Na}^{+}, \mathrm{K}^{+}, \mathrm{Ca}^{++}\right), z$ is its charge, and $X(i)$ refers to the $i^{t h}$ type of exchange site. In the Gaines-Thomas convention, each exchange site, $X(i)$ has a charge of -1 . The activities of adsorbed species correspond to the charge equivalent fractions, $\beta(i)_{M}$,

$$
\beta(i)_{M}=\frac{z_{M} q(i)_{M}}{\sum_{M} z_{M} q(i)_{M}}=\left\{X(i)_{M}\right\}
$$

where $z_{M}$ is the charge of cation $M, q(i)_{M}$ is the concentration of adsorbed cation $M$ in exchange site $i$ (moles/g), and the curly brackets denote activities. The Gapon activity convention is obtained by writing the reactions in every case with a single exchanger (Appelo and Postma, 1993). Alternatively, the Vanselow convention (Vanselow, 1932) describes the exchanger activity with mole fractions

$$
\beta(i)_{M}=\frac{q(i)_{M}}{\sum_{M} q(i)_{M}}=\left\{X(i)_{M}\right\} .
$$

The exchange reactions can then be used to write a mass action equation for binary Cs-M exchange:

$$
\begin{aligned}
K_{M / C s} & =\frac{\beta(i)_{M}^{1 / z}\left\{C s^{+}\right\}}{\beta(i)_{C s}\left\{M^{z+}\right\}^{1 / z}} \\
& =\frac{\left\{X(i)_{M}\right\}^{1 / z}\left\{C s^{+}\right\}}{\left\{X(i)_{C s}\right\}\left\{M^{z+}\right\}^{1 / z}} .
\end{aligned}
$$

In a single-site ion exchange model, the CEC is equal to the sum of the charge equivalent concentrations of the adsorbed cations:

$$
C E C=\sum_{M} z_{M} q_{M}
$$

while in a multi-site model, the CEC is the charge summed over all of the cation exchange sites (Cernik et al., 1996; Voegelin et al., 2000)

$$
C E C=\sum_{i} \sum_{M} z_{M} q(i)_{M}
$$

Assumptions For the following, it is assumed that the surface and aqueous species are in equilibrium. 
Surface Complexation. An alternative approach that allows a modeler to describe sorption while simultaneously considering variable chemical conditions in the subsurface is a surface complexation model (Davis et al., 2004). In this approach, the sorbing sediment surfaces are considered to possess surface functional groups that can form complexes analogous to the formation of aqueous complexes in solution. These surface reactions include proton exchange, cation binding and anion binding via ligand exchange at surface hydroxil sites (represented here as $\mathrm{XOH}$ to avoid confusion with other chemical species). For example, the sorption of a metal could be represented as

$$
X O H+M^{z_{+}} \leftrightharpoons X O M^{z_{+}-1}+H^{+}
$$

At equilibrium, the sorption reactions can be described by the mass law equation

$$
K_{a p p}=\frac{\left[X O M^{z_{+}-1}\right]\left\{H^{+}\right\}}{[X O H]\left\{M^{z+}\right\}},
$$

where $K_{a p p}$ is referred to as the apparent equilibrium constant, because it includes surface charge effects and hence is dependent on the extent of surface ionization (Dzombak and Morel, 1990), $\{i\}$ is the thermodynamic activity of aqueous species $i$, and the terms in square brackets represent the concentration of surface complexes $(\mathrm{mol} / \mathrm{kg})$.

Surface complexation differs from the simpler isotherm and ion-exchange models in several important ways. Surface complexation is based on the electrical double layer (EDL) theory. EDL theory assumes that the surface charge of a sorbent in contact with solution generates an electrostatic potential that declines rapidly away from the sorbent surface, creating an electrostatic field. An additional energetic term accounting for the work needed for the aqueous species to travel across the surface electric field is required:

$$
\begin{aligned}
\Delta G_{a d s} & =\Delta G_{i n t r}+\Delta G_{\text {coul }} \\
& =\Delta G_{\text {intr }}+\left(\Delta G_{\psi=0}-\Delta G_{\psi=\psi_{0}}\right) \\
& =\Delta G_{\text {intr }}-z F \psi_{0} .
\end{aligned}
$$

where $\Delta G_{a d s}$ is the free energy change of the overall adsorption reaction, $\Delta G_{\text {intr }}$ and $\Delta G_{\text {coul }}$ are the free energy change due to chemical bonding and to the electrostatic work (Coulombic attraction), respectively, $z$ is the charge of the surface species, $F$ the Faraday's constant (96485 $\mathrm{C} / \mathrm{mol})$, and $\psi_{0}$ is the mean surface potential $(V)$. Since

$$
\Delta G=-R T \ln K
$$

Equation (5.109) can be rewritten as

$$
K_{\text {app }}=K_{\text {int }} \exp \left(\frac{z F \psi_{0}}{R T}\right),
$$

where $R$ is the gas constant $(8.314 \mathrm{~J} / \mathrm{mol} / \mathrm{K}), T$ is the absolute temperature $(\mathrm{K})$, and $K_{\text {int }}$ is the intrinsic equilibrium constant which does not depend on the surface charge. 
Bulk and Mineral Specific Surface Complexation. There are two major approaches for applying the surface complexation concept to soils and sediments: the Component Additivity (CA) and Generalized Composite (GC) approaches (Davis et al., 1998, 2004). In the CA approach, it is assumed that a mineral assemblage is composed of a mixture of one or more reference phases, whose surface chemical reactions are known from independent studies of each phase (e.g. Arnold et al., 2001; Davis et al., 2004; Landry et al., 2009). Based on a measurement of the relative amounts or surface areas of each mineral present in the soil or sediment, sorption by the mixture of phases can be predicted by an equilibrium calculation, without any fitting of experimental data for the mixture. CA model predictions are sometimes made by assuming that one mineral component dominates sorption (Barnett et al., 2002; Davis et al., 2004; Payne et al., 2004; Zhang et al., 2009), allowing a straightforward equilibrium calculation, if the exposed surface area of that mineral component in the soil or sediment can be quantified.

In the GC approach, the surface of the mineral assemblage is considered too complex to be quantified in terms of the contributions of individual phases to sorption and/or that the contribution of individual components is not additive. The complexity is caused, in part, by the difficulties in quantifying the electrical field and proportions of surface functional groups at the mineral-water interface in the mixture of mineral phases and associated surface coatings. In the GC approach, it is assumed that sorption can be described by mass laws written with "generic" surface functional groups, with the stoichiometry and formation constants for each mass law determined by fitting experimental data for the mineral assemblage as a whole (Bond et al., 2008; Davis et al., 2004; Hyun et al., 2009). The GC modeling approach has generally been applied using a non-electrostatic model (NEM), which considers surface equilibria strictly as chemical reactions without explicit correction for electrostatic attraction or repulsion (Davis et al., 2004; Kent et al., 2000; Yabusaki et al., 2008). In an NEM, the apparent binding constants and stoichiometry of the mass action equations are derived by fitting the macroscopic dependence of adsorption as a function of $\mathrm{pH}$ (Davis et al., 1998). Because of the exclusion of electrical double layer terms, the mass action equations are not expected to provide accurate representations of the stoichiometry of the reactions at the molecular scale, however, the surface reactions can still be coupled with aqueous complexation reactions to provide simulations of macroscopic sorption as a function of aqueous chemical conditions.

Although there are differences between the GC and CA approaches, they are very similar with respect to their scientific basis. The following concepts form the basic tenets of both GC and CA modelling approaches (Davis et al., 1998):

1. Mineral surfaces are composed of chemical functional groups that can react with dissolved solutes to form surface complexes (coordinated complexes or ion pairs) in a manner analogous to aqueous complexation reactions in homogeneous solutions.

2. The equilibria of surface complexation and ionization reactions can be described via mass law equations, either with or without correction factors applied for electrostatic attraction to or repulsion from the surface.

3. The apparent binding constants determined for the mass law equations of surface complexation and ionization reactions are semi-empirical parameters related to thermodynamic constants via rational activity coefficients for surface species. 
Both CA and GC models may:

1. be coupled to the same critically reviewed aqueous thermodynamic data

2. use spectroscopic data to constrain and/or determine surface complex chemical composition and stoichiometry, and

3. use the same mass laws and surface species.

The differences among the model approaches lie primarily in the manner in which the models are calibrated and assumptions about various model parameters (in particular, whether the contributions of the various mineral phases to sorption and electrostatic fields can be considered as additive). CA models have almost always been applied using mass laws with electrostatic correction factors, while GC models have not usually used these factors.

Experimental and Modeling Issues Associated with SCMs for Soils and Sediments. Common to all applications of surface complexation approaches in soils and sediments is an initial characterization with respect to surface area, bulk mineralogy, and clay and organic carbon content. In addition, if the sediment is already contaminated with a metal or radionuclide, a measurement of the labile fraction of the contaminant needs to be determined (Bond et al., 2008; Curtis et al., 2004; Kohler et al., 2004).

In the GC approach, laboratory experiments are conducted with the field site sediments across the range of chemical conditions that are relevant to the scenarios of the physical and temporal modeling domains. Then, mass law relationships are derived that describe the change in metal or radionuclide sorption with variations in the aqueous chemical conditions (Davis et al., 2004). Total surface functional groups are typically estimated from surface area measurements. The number of surface site types and surface binding reactions is a practical modeling decision made based on the goodness-of-fit and the desired number of modeling parameters (Hyun et al., 2009).

In the CA modeling approach, after the sediment mineralogy is known, an estimate of the distribution of mineral surface areas is made. This can be done by simply assuming that the bulk weight abundances of various mineral phases are related to the distribution of functional groups at the sediment surface. For example, if quartz represents $60 \%$ by weight of the sediment, then an initial estimate could be that $60 \%$ of the surface area is represented by the quartz surfaces. Then a model of metal or radionuclide adsorption on quartz (as a function of chemical conditions relevant to the field site) is chosen from available literature data. Similar models for other minerals in the sediment are also catalogued. In some cases, model parameters may need to be re-derived from the original experimental data to develop a dataset that is self-consistent. In particular, this may be necessary if different electrical double layer models were used in the reference mineral models. Other approaches for estimating the distribution of mineral surface areas may be used, including chemical extractions and other methods (Davis et al., 1998, 2004). Once the component mineral models have been chosen, a predictive calculation of metal or radionuclide sorption for a specific set of chemical conditions can be made.

Possible limitations inherent to the surface complexation approach include poor representation of: a) surface functional groups, b) surface area, c) electrical double layer properties, d) surface 
species, e) surface binding constants, and f) competing surface reactions and their electrostatic effects. These limitations exist for both GC and CA modeling approaches, but the GC approach attempts to resolve some of the issues by using empirical data to overcome unknown factors and unmeasured parameters. For example, consider the representation of surface functional groups: Assume that only silanol, aluminol, ferrinol, and clay mineral edge sites are of importance in a particular sediment sample. At present it is very difficult or expensive to determine the distribution of mineral surface areas in a mixed mineral assemblage. Extractions, X-ray diffraction, and surface spectroscopies have been used by various investigators, but each of these methods provides estimates that are difficult to confirm independently. This uncertainty is circumvented in the GC approach by assuming that the distribution of site types is an unknowable quantity, and only generic sites are used. However, this requires that experimental data for the metal or radionuclide sorption on a site-specific sediment sample are collected, whereas in principle at least, additional characterization experiments are not needed for the CA modeling approach.

Quantifying Surface Sites Surface area is an important experimental quantity to be characterized in all surface complexation approaches. Typically a mixed mineral assemblage is characterized by BET analysis of nitrogen gas adsorption. Adjustments may need to be made for samples that contain high abundances of clay minerals, depending on whether there is evidence of sorption on the basal planes of clay mineral particles. Many investigators have concluded that surface functional groups of the basal planes are unreactive for metal and radionuclide sorption, and therefore the surface area of the basal planes does not need to be included in most applications. Fortunately, the BET method does not typically measure the surface area of the basal planes. In GC applications, the surface area is typically used in a straightforward manner to quantify the total abundance of surface sites using a conversion factor. In CA applications, however, the surface area should be distributed among different functional group types.

Multiple site-types are commonly used in formulating SCMs and approximate the nonlinear isotherms commonly observed for cation adsorption on well-characterized oxide mineral phases (Davis and Kent, 1990; Dzombak and Morel, 1990). Postulating multiple site-types is also important for simulating peak tailing observed in experimental studies of U(VI) transport in columns (Kohler et al., 1996). Reactive transport simulations that use multisite adsorption models can also simulate significant peak tailing in field-scale simulations (Curtis et al., 2006; Kent et al., 2000, 2008, 2007).

\section{Sub-models}

1. Non-electrostatic Models: EDL models differ in whether coulombic attraction or repulsion terms are considered in the mass laws of surface reactions. A non-electrostatic EDL means that the term

$$
\exp \left(\frac{z F \psi_{0}}{R T}\right)
$$

in Equation (5.111) need not be considered. While electrical double layer (EDL) models may represent these terms well for simple systems with single mineral phases, the approaches for treating these terms in mixed mineral assemblages have not been studied. In Component Additivity (CA) (Davis et al., 1998, 2004) applications to sediments, typically authors assume that the EDL properties of pure, clean mineral phases investigated remain the same 
in mixed mineral assemblages (Davis et al., 2004). This ignores the likely effects of surface contaminants (adsorbed major solutes such as silicate, organic compounds, etc.) and the overlapping EDL regions of particles that are known to change coulombic terms. In Generalized Composite (GC) approaches (Davis et al., 1998, 2004), the coulombic attraction or repulsion terms are not included, but are instead built into the semi-empirical model calibration of reaction stoichiometries and binding constants to experimental data. That is, whatever EDL forces exist, they are lumped into the model fitting of reactions and binding constants. In each case, there is inherent uncertainty in the modeling approach. The errors within the GC model may not be that significant because of model calibration to experimental data, but the error is only minimized by confining model calculations to chemical conditions interpolated between those investigated in laboratory experiments. Extrapolation of any non-electrostatic model to uninvestigated chemical conditions is unwise because the EDL forces for those conditions will not necessarily be captured accurately by the model calibration. In addition the formation of unknown surface species may not be realized if calculations are extrapolated to chemical conditions not investigated at all.

2. Electrostatic models: When the coulombic attraction or repulsion terms is considered as shown in Equation (5.111), the electrostatic models differs also among themselves in how they conceptualize the structure of the double-layer and describe changes in surface potential and surface charge from the surface of the sorbent phase to the bulk solution. In the constant capacity and diffuse-layer models, all adsorbed species are considered specifically adsorbed at the zero plane while the triple layer model can assign adsorbed species to either a zero plane or more distant plane. The constant capacity and diffuse-layer model are elaborated in the following sections.

(a) Constant Capacitance The constant capacitance model is a special case of the diffuselayer model. Both models are based on the assumption that all the species are adsorbed in the same layer and a diffuse layer of counterions constitutes the transition to homogenous solution. Additionally, it is assumed that the surface potentials are small, or the double layer has been compressed (very high ionic strength). However, differently from the diffuse-layer model, the relationship between the surface charge and the potential is assumed to be linear:

$$
\sigma=\mathbb{C} \psi,
$$

where $\sigma$ is the surface charge, $C m^{-2}, \psi$ is the potential at the surface, $V$, and $\mathbb{C}$ is a constant capacitance value, $C V^{-1} \mathrm{~m}^{-2}$, to be obtained from fitting experimental data. Equation (5.113) is solved for the potential and substituted into Equation (5.111).

(b) Diffuse Double Layer Model The diffuse layer model has been described in great detail by Dzombak and Morel (1990) and was applied to adsorption of metals on iron oxide surfaces. In the diffuse layer model, the solid-water interface is composed of two layers: a layer of surface-bound complexes and a diffuse layer of counter ions in solution. The surface charge is calculated from the total surface species adsorbed on 
the layer:

$$
\sigma_{p}=\frac{F}{A} \sum_{k=1}^{N_{s}} z_{k} y_{k}
$$

Here $A$ is the surface area sorbent per liter solution $\left(m^{2} / L\right), F$ is the Faraday constant $(96,480 \mathrm{C} / \mathrm{mol}), z_{k}$ is the charge of the ion, and $y_{k}$ is the concentration $(\mathrm{mol} / \mathrm{L})$ of surface bound ions in the Stern Layer. According to the Gouy-Chapman theory, the surface charge density $\sigma_{p}\left(C / \mathrm{m}^{2}\right)$ is related to the potential at the surface (volts) by:

$$
\sigma_{p}=\left(8 R T \epsilon_{R} \epsilon_{0} C_{e} \times 10^{3}\right)^{1 / 2} \sinh \left(\frac{z F \psi_{0}}{2 R T}\right)
$$

where $R$ is the molar gas constant (8.314 $\left.\mathrm{Jmol}^{-1} \mathrm{~K}^{-1}\right), C_{e}$ is the molar electrolyte concentration $(M), z$ is the electrolyte charge, $T$ is the absolute temperature $(K), \epsilon_{R}$ is the relative dielectric constant of water $\left(\epsilon=78.5\right.$ at $\left.25^{\circ} C\right)$, and $\epsilon_{0}$ is the permittivity of free space $\left(8.854 \times 10^{-12} C V^{-1} m^{-1}\right)$. Equation (5.115) is only valid for a symmetrical electrolyte, the anion and cation must have the same charge. Note that $C$ the unit (coulombs or celcius) is not a concentration. Capacitance is not solved for explicitly, but is implicitly accounted for in Equation (5.115). It is common to use the linearized version of Equation (5.115) for low values of the potential:

$$
\sigma_{p}=\epsilon \epsilon_{0} \kappa \psi_{0}
$$

where $1 / \kappa(\mathrm{m})$ is the double-layer thickness defined as

$$
\frac{1}{\kappa}=\left(\frac{\epsilon \epsilon_{0} R T}{2 F^{2} \cdot 1000 I}\right)^{1 / 2},
$$

where $I$ is the ionic strength $m o l L^{-1}$. The first term of Equation (5.115), $\left(8 R T \epsilon \epsilon_{0} C_{e} \times\right.$ $\left.10^{3}\right)^{1 / 2}$, can be rewritten at $25^{\circ} \mathrm{C}$ :

$$
\sigma_{p}=0.1174 C_{e}^{1 / 2} \sinh \left(\frac{z F \psi_{d}}{2 R T}\right) .
$$

Therefore, in the diffuse-layer model, the value of the capacitance $\mathbb{C}$ relating the surface charge and the potential can be calculated based on theoretical considerations instead of being an experimental fitting parameter.

(c) Triple Layer Model The triple layer model is similar to the double layer model, but divides the sorbed species into two layers, Figure 2. Strongly sorbed species are located close to the surface, the zero plane, while weakly sorbed species reside in the beta plane, seperated from the surface by the strongly sorbed species and hydration layers (e.g. Langmuir, 1997). Further out from the surface is a diffuse layer and the bulk solution similar to the double layer.

The charge balance equation for the triple layer model is

$$
\sigma_{0}+\sigma_{\beta}+\sigma_{d}=0
$$


where $\sigma_{0}, \sigma_{\beta}$ and $\sigma_{d}$ are the net surface charges in the zero, beta and diffuse planes respectively, $\left(C / \mathrm{m}^{2}\right)$. The net surface charge in the zero plane is given by:

$$
\sigma_{0}=\frac{F}{A} \sum_{k=1}^{N_{s}} z_{k} y_{k}^{0},
$$

where the variables are as defined in Equation (5.114) with the exception of $y_{k}^{0}$, which is the concentration $(\mathrm{mol} / \mathrm{L})$ bound in the zero plane. Similarly, the net surface charge of the beta plane is

$$
\sigma_{\beta}=\frac{F}{A} \sum_{k=1}^{N_{s}} z_{k} y_{k}^{\beta},
$$

where $y_{k}^{\beta}$ refers to the ions bound in the beta plane. Note that the composition of the diffuse layer is not often calculated explicitly in either Triple Layer Model or the Diffuse Double Layer Model, although a method to do so has been presented by Leroy et al. (2007).

The triple layer model assumes constant capacitances between the zero plane and beta plane, $\mathbb{C}_{1}$, and the beta plane and d-plane, $\mathbb{C}_{2}$. These are related to the surface charges and potentials by:

$$
\begin{gathered}
\sigma_{0}=\mathbb{C}_{1}\left(\psi_{0}-\psi_{\beta}\right) \\
\sigma_{\beta}=\mathbb{C}_{1}\left(\psi_{\beta}-\psi_{0}\right)+\mathbb{C}_{2}\left(\psi_{\beta}-\psi_{d}\right) \\
\sigma_{d}=\mathbb{C}_{2}\left(\psi_{d}-\psi_{\beta}\right) .
\end{gathered}
$$

\subsubsection{Common Data Needs for Sorption Models}

All sorption models will require access to a database of parameter values that are potentially independent of the specific contaminated site under consideration. For example, the cation exchange capacity (CEC) of a mineral like smectite or kaolinite can be described with a range of values. However, it is likely that site-specific experimental data will have to be collected and either collected in a site-specific database, or serve as the basis of a site-specific lookup table.

\subsection{Mineral Precipitation and Dissolution}

\subsubsection{Overview}

Mineral precipitation and dissolution are among the most important processes affecting the transport of contaminants in the subsurface. They represent a class of heterogeneous reactions that require a slightly different treatment than do reactions taking place within the same phase. Perhaps most importantly, a kinetic treatment of mineral reactions requires the inclusion of the interfacial 


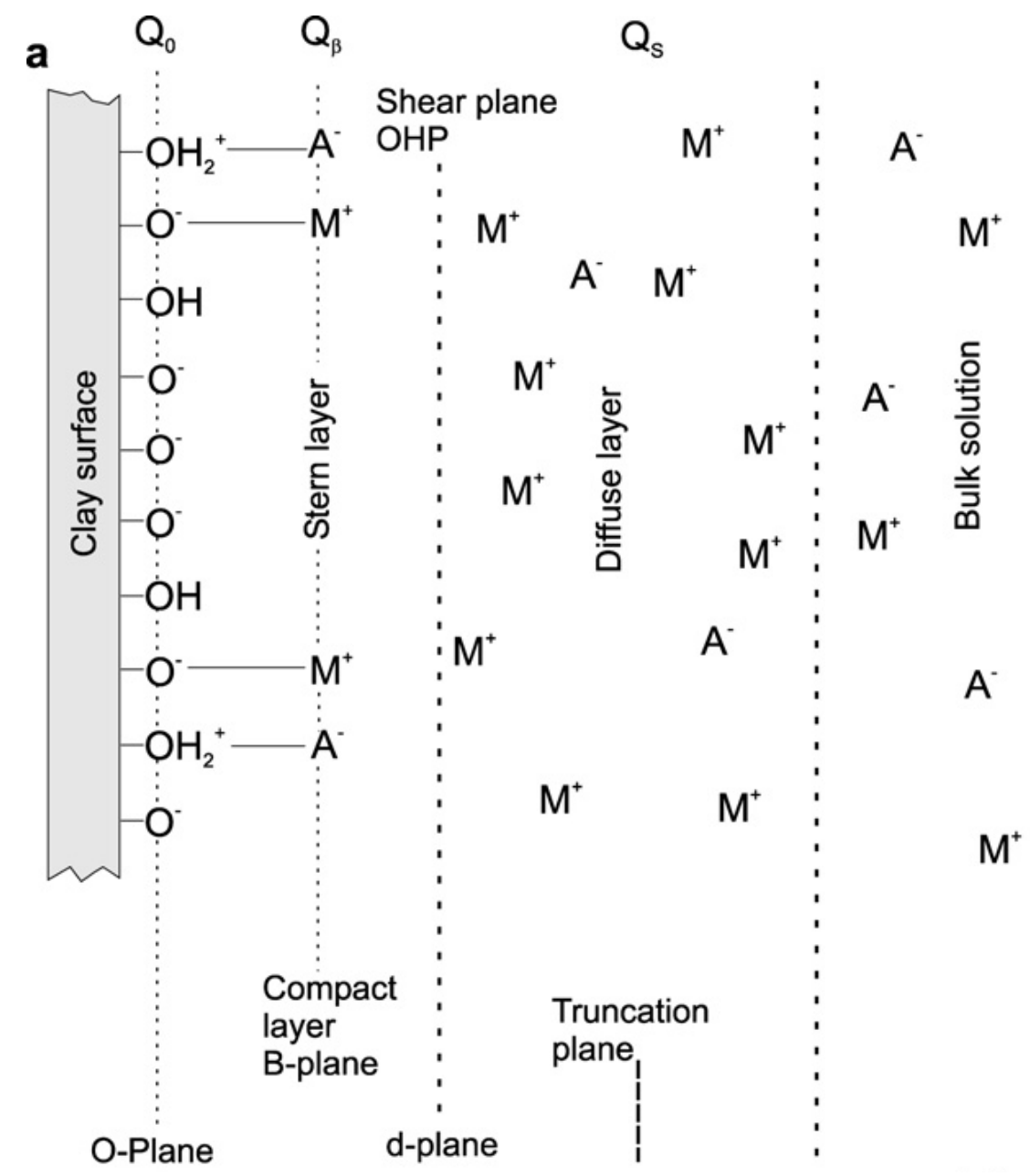

b

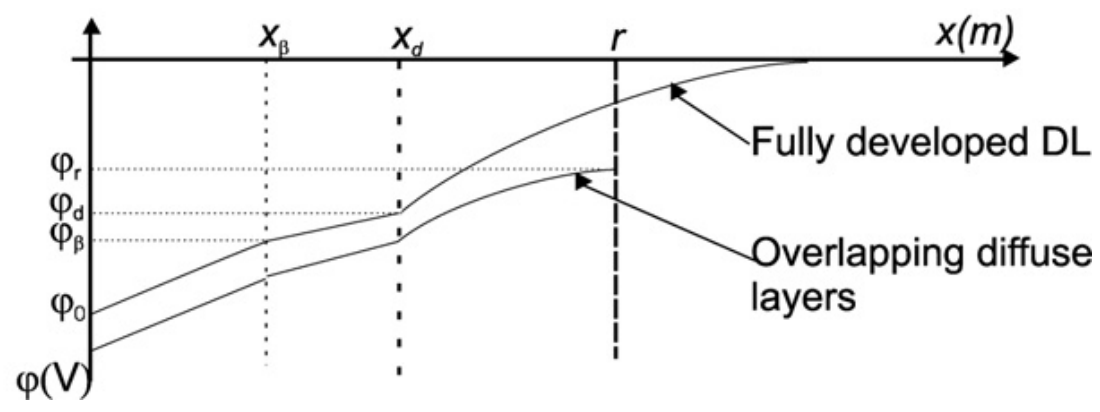

Figure 2: Schematic of the TLM model from Gonçalvès et al. (2007). 
area between the phases (water and mineral), or reactive surface area (see Section 5.4.5). The reactive minerals may be considered as pure, in which case their treatment is simplified by the fact that their activity is always $=1$, or they may be solid solutions, in which case their activities have to be determined as in any other solution. Minerals may be assumed to be at equilibrium with the aqueous solution, in which case they can be included in the total concentration in a fashion similar to the way in which equilibrium secondary species are (Equation (5.5)), or they may be treated kinetically. In most cases, it appears to be sufficient to treat the minerals kinetically, since the equilibrium condition can be regained by using reaction rates that are sufficiently fast relative to the time scales of interest (Steefel and MacQuarrie, 1996). This approach also offers the advantage that the minerals can potentially be removed as direct unknowns in the solution procedure within any one nonlinear iteration cycle and updated only at the end of the time step.

\subsubsection{Kinetic Mineral-Water Rate Laws}

Following the notation presented in Section 2, the mineral reactions take the form

$$
\sum_{j} \nu_{j m} \mathcal{A}_{j \alpha} \rightleftharpoons \mathcal{M}_{m}
$$

for mineral $\mathcal{M}_{m}$ with reaction rate $I_{m \alpha}$ and stoichiometric coefficients $\nu_{j m}$. The sum of the mineral reaction rates affecting component $j$ in phase $\alpha$ can be written as

$$
R_{j \alpha}=\sum_{m} \nu_{j m} I_{m \alpha}
$$

In most cases, this will involve water as the fluid phase. Equation (5.126) implies that component $j$ may be involved in any number of parallel mineral reaction pathways (even within the same phase), with each potentially described by its own rate law. Changes in mineral concentrations are described by the equation

$$
\frac{\partial \phi_{m}}{\partial t}=\bar{V}_{m} \sum_{\alpha} I_{m \alpha},
$$

with molar volume $\bar{V}_{m}$ and where the sum over $\alpha$ on the right-hand side is over all fluid phases that react with the $m$ th mineral.

We use a kinetic rate law based on the assumption that attachment and detachment of ions from mineral surfaces is the rate-limiting step (i.e., a surface reaction-controlled rate law). It does not mean, however, that one cannot obtain overall transport control on the mineral dissolution or precipitation rate since this depends on the magnitude of the reaction rate relative to the macroscopic transport rates. The rate laws used for mineral precipitation and dissolution are based loosely on transition state theory (Aagaard and Helgeson, 1982; Lasaga, 1981, 1984)).

TST Type Rate Law. This formulation gives the dependence of the rate on the saturation state of the solution with respect to a particular mineral as a function of the ion activity product, $Q_{s}$, defined by

$$
Q_{m}=\prod_{j=1}^{N_{c}} a_{j}^{\nu_{j m}}
$$


where the $a_{j}$ are the activities of the primary species used in writing the dissolution reaction for the mineral and $\nu_{j m}$ are stoichiometric reaction coefficients. In order to incorporate the strong $\mathrm{pH}$ dependence of most mineral dissolution and precipitation reactions far from equilibrium, parallel rate laws are used which are summed to give the overall reaction rate law for a particular mineral in phase $\alpha$

$$
I_{m \alpha}=-A_{m \alpha}\left\{\sum_{l=1}^{N_{r m}} k_{l}\left(\prod_{i=1}^{N_{c}+N_{x}} a_{i}^{p_{i l}}\right)\left[1-\left(\frac{Q_{m}}{K_{m}}\right)^{M_{l}}\right]^{n_{l}}\right\},
$$

where $k_{l}$ is the far from equilibrium dissolution rate constant for the $l$ th parallel reaction, $p_{i l}$ is the exponential dependence on species $i$ of the $l$ th parallel reaction (i.e., the reaction order), $K_{m}$ is the equilibrium constant, $N_{r m}$ is the number of parallel reactions within phase $\alpha$, and $A_{m \alpha}$ refers to the surface area of the reacting mineral in contact with phase $\alpha\left(\mathrm{m}^{2}\right.$ mineral $\mathrm{m}^{-3}$ porous medium). The exponents $n_{l}$ and $M_{l}$ allow for nonlinear dependencies on the affinity term and are normally taken from experimental studies. The term $\prod_{i=1}^{N_{c}+N_{x}} a_{i}^{p_{i l}}$ incorporates the effects of various ions in solution on the far from equilibrium dissolution rate. This is most commonly the solution $\mathrm{pH}$ or hydroxyl ion activity but may include other electrolytes as well.

The temperature dependence of the reaction rate constant can be expressed reasonably well via an Arrhenius equation (Lasaga, 1984). Since many rate constants are reported at $25^{\circ} \mathrm{C}$, it is more convenient to write the rate constant at some temperature as

$$
k=k_{25} \exp \left[\frac{-\mathrm{E}_{\mathrm{a}}}{\mathrm{R}}\left(\frac{1}{\mathrm{~T}}-\frac{1}{298.15}\right)\right],
$$

where $E_{a}$ is the activation energy, $k_{25}$ is the rate constant at $25^{\circ} \mathrm{C}, R$ is the gas constant, and $T$ is temperature in the Kelvin scale.

Nonlinear Parallel Mineral Rate Laws. The rate law proposed by Hellmann and Tisserand (2006), based on experimental data for albite, can be used for dissolution of silicate minerals. One rate law describes far from equilibrium dissolution behavior with a rate constant $k_{2}$, and one rate law describes close to equilibrium behavior $\left(k_{1}\right)$ :

$$
I_{m \alpha_{\circ}}=A_{m \alpha_{\circ}}\left\{k_{1}\left[1-\exp \left(-m_{1} g^{m_{2}}\right)\right]+k_{2}[1-\exp (-g)]^{m_{3}}\right\},
$$

where $g$ represents $\frac{\left|\Delta G_{r}\right|}{R T}$ and the fitted parameters $m_{1}, m_{2}$ and $m_{3}$ have values of $7.98 \times 10^{-5}, 3.81$ and 1.17 (Hellmann and Tisserand, 2006). Here again the assumption is that the phase in question, $\alpha_{\circ}$, is water. This formulation is consistent with theoretical and experimental considerations which suggest that far-from-equilibrium dissolution is characterized by the opening of etch pits and rapid propagation of step waves, whereas close-to-equilibrium dissolution in the absence of etch pits is localized to surface defects.

Dissolution Only. The simplest form of a dissolution only rate law would be a completely irreversible reaction with no back reaction (i.e., no precipitation). However, it may be desirable to have a rate law which slows as equilibrium is approached, even though the back reaction cannot really be demonstrated. Such a rate law is likely applicable to the dissolution of albite at low temperature, since dissolution can be demonstrated while precipitation cannot. There is clear evidence in the 
case of plagioclase that the rate of dissolution does slow, so it is important to be able to include this in the code (Maher et al., 2009). Similarly, it was found that kaolinite could not be described with a single rate law that was continuous for both dissolution and precipitation (Yang and Steefel, 2008). To describe both precipitation and dissolution of kaolinite, therefore, one can use distinct dissolution-only and precipitation-only rate laws.

A rate law for dissolution only could in principle include any number of rate laws having a TST (linear or nonlinear) form, but with the added code (here presented as a linear TST rate with no dependence on dissolved or sorbed species far from equilibrium for the sake of simplicity):

$$
I_{m \alpha_{\circ}}= \begin{cases}-A_{m \alpha_{\circ}}\left[1-\left(\frac{Q_{m}}{K_{m}}\right)\right] & \text { if } I_{m \alpha_{\circ}}<0, \\ 0 & \text { if } I_{m \alpha_{\circ}}>0 .\end{cases}
$$

Precipitation Only. A precipitation-only rate law takes a similar form to that of dissolution-only

$$
I_{m \alpha_{\circ}}= \begin{cases}A_{m \alpha_{\circ}}\left[1-\left(\frac{Q_{m}}{K_{m}}\right)\right] & \text { if } I_{m \alpha_{\circ}}>0 \\ 0 & \text { if } I_{m \alpha_{\circ}}<0 .\end{cases}
$$

\subsubsection{Assumptions and Applicability for Rate Laws}

All of the rate laws described above use reactive surface area as an important parameter (see Section 5.4.5). This is because most of the rates determined for mineral dissolution and precipitation are based on normalization to physical surface area. Rate laws that consider the actual kind and density of reactive sites are possible, but so far are difficult to implement at the field scale.

\subsubsection{Data Needs for Rate Laws}

Data needs for mineral dissolution and precipitation are considerable and help to explain why these processes have not always been included in subsurface environmental management codes. In the case of mineral dissolution, it is necessary to know the reactive surface area of the dissolving mineral in contact with the mobile fluid phase. Reactive surface area within immobile zones may contribute to the reactivity as well over long time scales via diffusion, so normally must be considered as well (see Section 5.4.5).

Reactive surface area is an even more difficult topic in the case of mineral precipitation. Here seeds may be created by nucleation, the seeds may growth via crystal growth and/or ripening and agglomeration (Steefel and Van Cappellen, 1990). Some proposed methods for including the evolution of reactive surface area are given in Section 5.4.5.

\subsubsection{Reactive Surface Area Evolution}

Surface area is a key parameter affecting mineral dissolution and precipitation rates, as well as the extent of aqueous species (e.g., contaminants) sorption onto mineral surfaces. Accordingly, surface area is one of the variables that appear in mineral dissolution and precipitation rate laws, 
Section 5.4, as well as in expressions needed to compute sorption site concentration for surface complexation models, Section 5.3.2. The incorporation and treatment of surface areas into reactive transport simulations can be broken down into two parts: initial surface areas, Equation (5.134), which can be either directly input into the model if known, or estimated from input geometric data and Equation (5.138) the actual evolution of surface areas (starting from input or calculated initial values) upon mineral dissolution or precipitation.

Initial surface areas can be estimated from laboratory measurements for pure minerals or bulk sediments. However, actual "reactive" surface areas in natural systems are largely unknown, and have been shown to be typically smaller than laboratory measurements by several orders of magnitude, and in much closer agreement with geometric mineral surface areas. For this reason, it is not uncommon to estimate initial reactive surface areas from available geometric data on the size and shape of mineral grains in porous media, or from data on fracture coverage (thus spacing) in fractured rocks. This can be achieved either internally or externally prior to input, using relatively simple mathematical expressions that do not require a high level of accuracy given the large variability of this parameter in natural systems. Alternatively, initial surface areas can be calibrated during the course of reactive transport simulations.

Once initial (reactive) surface areas have been determined, the evolution of these areas upon mineral reaction needs to be captured in a manner that is consistent with field and experimental observations. For dissolving minerals in water-saturated systems, the evolution of reactive surface area can be calculated, as a first approximation, by assuming some proportionality between the amount of mineral present and its surface area. In such case, simple relationships can be developed relating surface area with mineral volume fraction, as shown further below. In unsaturated systems, however, the problem is complicated by the fact that reactive surface areas are not only function of mineral volume fractions, but also potentially of liquid saturation. While water serves as the wetting phase in most cases, and thus in in contact with the solid grains in the medium, at low saturations the coverage may become discontinuous. In this case, as a first approximation, the reactive surface area in contact with the phase (in the case of water, the "wetting phase") can be assumed to be proportional to liquid saturation.

Predicting the evolution of surface area from the onset of, and during, mineral precipitation is less straightforward. If a mineral forms on existing surfaces (of the same mineral and/or on surfaces of existing precursors), the surface area can be assumed to evolve with some proportionality to the current volume fraction of the mineral (or precursor mineral(s)). However, if a mineral actually nucleates from solution, without precursors, a rigorous treatment of nucleation is required (Steefel and Van Cappellen, 1990). Such rigorous treatment, however, is deemed outside the scope of current model requirements, primarily because input parameters for nucleation models are scarce for most minerals. Instead, an approximate treatment can be considered, yielding a trend of surface area evolution similar to that expected upon nucleation (i.e., initially large surface areas upon nucleation decreasing with growth). This general behavior can be captured by assuming that the initial (first formed, minimum) amount and grain size of a nucleating mineral is known. Using these two (input) parameters (i.e., minimum/initial volume fraction and grain size), the initial number of precipitating mineral grains and their surface area can be easily computed for each mineral assuming simple grain geometries (e.g., spheres). Upon further precipitation, the evolution of surface area can then be computed as a function of mineral grain size, with mineral grains growing with some proportionality to the amount of mineral precipitation. As such, surface areas initially 
decrease with increasing mineral amounts, starting from initially large values at small initial grain sizes. For each mineral, this decrease in surface area with growth can be assumed to continue until the surface area reaches some preset (input) value corresponding to the surface area of the "bulk" mineral. At this point, the surface area is assumed to evolve again with some direct proportionality to volume fraction, as in the case of dissolving minerals.

The general methodology and formulation of the above-described approach are presented further below. Note that because surface areas evolve relatively slowly in most systems, compared to other parameters such as aqueous concentrations, surface areas can be computed explicitly. That is, surface areas computed at the end of a flow/transport/reaction time step can be used as values for computing reactive transport at the next time step.

Reactive Surface Area. The following general relationship can be used to compute reactive surface areas of minerals as a function of time:

$$
A_{m \alpha}=\gamma_{m}\left(\phi_{m} A_{S S_{m}} 1000 \rho_{m}+\bar{A}_{m \alpha}\right)
$$

where $A_{m \alpha}$ is the effective reactive surface area of minerals $\left(\mathrm{m}^{2}\right.$ mineral per $\mathrm{m}^{-3}$ porous medium), $\gamma_{m}$ is the fraction of the mineral's surface area that is in contact with the phase (normally water), $\phi_{m}$ is the volume fraction of the mineral, $A_{S S_{m}}$ is the specific surface area of the mineral $\left(\mathrm{m}^{2} / \mathrm{g}\right)$, $\rho_{M}$ is the dry density of the mineral $\left(\mathrm{kg} \mathrm{m}^{-3}\right)$, and the factor of 1000 converts from $\mathrm{kg}$ to $\mathrm{g}$. $\bar{A}_{m \alpha}$ is the precursor surface area $\left(\mathrm{m}^{2}\right.$ mineral $\mathrm{m}^{-3}$ medium $)$. The fraction of the mineral surface area, $\gamma_{m}$, in contact with the phase $\alpha$ may be estimated from petrographic observations, fitted from field data, or potentially estimated based on as yet unspecified relationship with phase (liquid) saturation.

An alternative expression for computing reactive surface area is given by Steefel and Lasaga (1994)

$$
A_{m \alpha}=\gamma_{m} A_{m \alpha}^{\circ}\left(\frac{\phi_{m}}{\phi_{m}^{\circ}}\right)
$$

where $A_{m \alpha}^{\circ}$ and $\phi_{m}^{\circ}$ are the initial surface area and volume fraction of the mineral, respectively.

In the case of secondary minerals that are not initially present and where no precursor mineral occurs with a non-zero volume fraction, both Eqns. (5.134) and (5.135) can be modified to include a "threshold" mineral volume fraction that is used just for the purposes of calculating reactive surface area. This mineral mass is considered to be derived from a short-lived nucleation event that quickly creates surface area upon which subsequent mineral growth can occur. The threshold volume fraction, $\phi_{\text {nucl }}$, can be incorporated in the following way:

$$
A_{m \alpha}= \begin{cases}\gamma_{m}\left(\phi_{m} A_{S S_{m}} 1000 \rho_{m}\right) & \text { if } \phi_{m}>\phi_{m}^{\text {nucl }} \\ \gamma_{m}\left(\phi_{\text {nucl }} A_{S S_{m}} 1000 \rho_{m}\right) & \text { if } \phi_{m}<\phi_{m}^{\text {nucl }}\end{cases}
$$

Such a procedure obviates the need for a more complicated formulation such as that found in Steefel and Van Cappellen (1990).

Another option to be implemented involves a simple geometric method for calculating surface area (Lasaga, 1984). If a simple cubic packing of spherical grains of radius $r$, is considered, then the cubic arrangement of spheres yields, in a cube of side $4 r$ and volume $(4 r)^{3}$, a total of 8 spheres, 
each of radius $r$, volume $\frac{4 \pi r^{3}}{3}$, and area $4 \pi r^{2}$. Thus the surface area $A_{n u c l}$ (as the area of the spheres divided by the volume of the cube) can be computed as

$$
A_{m \alpha}=\gamma_{m} \frac{0.5}{r}
$$

where $r$ is the average grain size of the mineral. A more comprehensive approach involving crystal size distributions has been proposed by Steefel and Van Cappellen (1990).

Estimation of Reactive Surface Areas for Fractures. In a dual permeability (fracture-matrix) system, the surface area of the fracture in contact with the mobile fluid phase, $A_{F}$ (in units of $\mathrm{m}^{2}$ fracture $\mathrm{m}^{-3}$ medium) is (Steefel and Lasaga, 1994)

$$
A_{F}=\varphi_{F} \frac{2}{\delta},
$$

where $\varphi_{F}$ is the fracture porosity and $\delta$ is the fracture aperture. To calculate the amount of mineral surface area present along the fracture, one can use the volume fraction of the primary dissolving phase as an estimate of the fraction of the fracture surface made up of that mineral

$$
A_{m \alpha}=\varphi_{F} \phi_{m} \frac{2}{\delta}
$$

For precipitation, various schemes are possible. If the assumption is made that mineral precipitation can occur anywhere along the fracture surface, then (5.138) can be used without modification. For partially wetted fractures, a correction can be introduced to reduce the reactive surface area:

$$
A_{m \alpha}=\varphi_{F} \gamma_{m} \phi_{m} \frac{2}{\delta}
$$

where $\gamma_{m}$ is the fraction of the fracture actually in contact with the reactive phase (normally water).

\subsection{Microbially-Mediated Reactions}

\subsubsection{Overview}

Microbially-mediated reactions or reaction networks represent an important class of biogeochemical processes that need to be incorporated into environmental management codes (Roden, 2008). Modeling of microbially-mediated processes is still in its infancy at this stage, however, with relatively little treatment of the actual metabolic processes of microbial communities, or the interaction of individual microbes making up the community. The revolution associated with the application of molecular genetic tools (Banfield et al., 2005), which aims ultimately provide quantitative markers for explicit metabolic pathways, is as yet still largely qualitative. As a result, the available approaches are limited in the extent to which they can capture the effects of environmental influences on metabolic rates. This in turn limits the ability to quantify biogeochemical reaction processes affecting contaminant transport.

As currently implemented in a number of reactive transport codes, microbially-mediated processes are approached from a macroscopic point of view, with the rate of a specific biogeochemical reaction (e.g., Fe(III) reduction) mediated by a microbial community. A number of biogeochemical 
models have been proposed that do not take explicit account of the microbial community dynamics, with the argument that under many conditions the population will evolve to some steady state value where its effects can be folded into the rate constant for the pathway (sometimes referred to as a biomass-implicit approach, e.g., Dale et al. 2006). This may often be the case in subsurface systems involving natural attenuation, but is clearly not a good assumption in the case of biostimulation experiments where the biomass and its metabolic activity involve over relatively short time scales (Li et al., 2009). In biogeochemical systems where biomass growth or death is important, it is important to be able to track the amount of carbon converted to biomass (anabolic pathways) as well as the amount of carbon used for respiration (catabolic pathways). As the relative proportion of these pathways change, the reaction stoichiometry must change as well in order to conserve mass locally and globally.

\subsubsection{Process Model Equations}

A variety of kinetic expressions have been used to describe microbially-mediated biogeochemical reactions, ranging from zeroeth-order kinetic schemes, to first-order schemes, to hyperbolic formulations (Michaelis-Menten or "Monod" kinetics). In addition, thermodynamic controls may be important as well, since the microbes can make use of only the energy $(\Delta G)$ that is available from the reaction, and perhaps not even as much as that since there is a minimum Gibbs energy necessary for maintenance of the ATP cycle (Jin and Bethke, 2005). However, a general formulation for a microbially-mediated reaction can be written (Dale et al., 2006)

$$
R_{m}=-\frac{v_{\max } B}{\Upsilon} \prod_{i} F_{i}
$$

where $R_{m}$ is the microbially mediated reaction rate $\left(m o l m^{-3} s^{-1}\right), v_{\max }$ is the maximum rate of substrate utilization $\left(s^{-1}\right), B$ is the microbial biomass (cells $m^{3}$ ), $\Upsilon$ is the cell yield (cells $\mathrm{mol}_{\text {substrate }}^{-1}$ ), and $F_{i}$ are dimensionless scaling functions for factors affecting growth. An alternative model is the non-interacting model (Bäder, 1978) assuming that the limiting factors for microbial growth are independent:

$$
R_{m}=-\frac{v_{\max } B}{\Upsilon} \min _{i} F_{i}
$$

where min is the minimum of all the possible limiting functions, $F_{i}$.

Monod Kinetics. The kinetic factor, $F_{K}$ is given by a standard dual Monod expression

$$
F_{K}=\left(\frac{\left[C_{D}\right]}{K_{D}+\left[C_{D}\right]}\right)\left(\frac{\left[C_{A}\right]}{K_{A}+\left[C_{A}\right]}\right)
$$

where $C_{D}$ and $C_{A}$ refer to the activities of the electron donor and acceptor respectively and $K_{D}$ and $K_{A}$ are the half-saturation constants for the electron donor and acceptor respectively.

It has been noted that energetically less favorable pathways are inhibited, so the Monod expressions also need to include hyperbolic inhibition terms of the form

$$
F_{K}=\left(\frac{\left[C_{D}\right]}{K_{D}+\left[C_{D}\right]}\right)\left(\frac{\left[C_{A}\right]}{K_{A}+\left[C_{A}\right]}\right)\left(\frac{K_{I}}{K_{I}+\left[C_{I}\right]}\right),
$$


where $K_{I}$ is the inhibition constant for a particular pathway by another species (normally an electron acceptor) and $C_{I}$ is the concentration of the inhibiting species for a particular pathway (e.g., $\mathrm{O}_{2}$ for a nitrate-reducing pathway).

Zeroeth-Order Kinetics. Zeroeth-order kinetics have been used in some cases to describe microbiallymediated reactions.

$$
R_{m}=v_{0},
$$

where $R_{m}$ is the substrate consumption rate and $\zeta$ is the rate constant with biomass implictly accounted for, moles $\mathrm{m}^{-3} \mathrm{~s}^{-1}$. Such formulations, however, do not allow for a decrease in rate as either equilibrium is approached or as the reactants are depleted, so they are inherently unrealistic and problematic in a numerical context. Monod kinetics do show zeroeth-order kinetics when electron acceptor and donor concentrations are sufficiently high, but the Monod formulations transition to first-order kinetics as concentrations of the acceptor and/or donor decrease.

First-Order Kinetics. Some classes of microbially-mediated reactions can be described with first-order kinetics, with perhaps the oxidation of organic matter being the most common of these. Such first-order biodegration schemes are relatively common in modeling organic matter in marine sediments (Berner, 1977) and in contaminant plumes dominated by organics (Lorah and Olsen, 1999). The first-order rate expression can be written (similarly to those for radioactive decay) with a dependence on either the electron donor or acceptor

$$
R_{m}=v_{1} C
$$

where $v_{1}$ is the first order rate constant for substrate consumption, $s^{-1}$ with implicit biomass.

Thermodynamic Factor. The thermodynamic driving force, which is assumed to drive the reaction in only one direction, is given by (Jin and Bethke, 2005)

$$
F_{T}=\max \left[0,\left(1-\exp \left(\frac{\Delta G_{n e t}}{\chi R T}\right)\right)\right],
$$

where $\Delta G_{N E T}$ is the fraction of the Gibbs energy of catabolism that provides a thermodynamic drive for the reaction (further defined below), $\chi$ is the average stoichiometric coefficient, $\mathrm{R}$ is the gas constant, and $\mathrm{T}$ is the absolute temperature. The average stoichiometric coefficient, $\chi$, is equivalent to the number of protons translocated across the cell membrane during catabolism and is assumed equal to 1 in anaerobic metabolism (Jin and Bethke, 2005). As is apparent from Equations (5.143) and (5.147), both $F_{K}$ and $F_{T}$ are dimensionless and vary from 0 to 1 . As pointed out, inclusion of such a thermodynamic term removes the need for kinetic inhibition terms as are often found in microbial rate models (Dale et al., 2008). A temperature dependence can be added through parameterization of the Gibbs energy (or equilibrium constants) in terms of temperature.

In the formulation of Dale et al. (2008) as adapted from Jin and Bethke (2005), the $\Delta G_{N E T}$ term is the sum of two Gibbs energy terms of opposite sign

$$
\Delta G_{N E T}=\Delta G_{I N S I T U}+\Delta G_{B Q}
$$


where $\Delta G_{I N S I T U}$ is the in situ Gibbs energy yield of the catabolic process and $\Delta G_{B Q}$ is the bioenergetic energy quantum (Dale et al., 2006; Hoehler, 2004)

$$
\Delta G_{I N S I T U}=\Delta G^{0^{\prime}}+R T \ln [Q] .
$$

Here $\Delta G^{0^{\prime}}$ is the standard Gibbs energy of catabolism at the in situ temperature. The minimum bioenergetic energy, $\Delta G_{B Q}$, is the minimum that can be exploited by living cells to synthesize adenosine tri-phosphate (ATP) and is coupled to the transfer of 3-4 protons across the cellular membrane.

Biomass Evolution. For microbially-mediated reactions, typically some portion of the organic matter (in the case of heterotrophs) or $\mathrm{CO}_{2}$ (in the case of autotrophs) is used for cell synthesis (Roden, 2008), with the remaining portion of the electrons are used to generate energy. The fraction of an electrons from the electron donor substrate used for cell synthesis is given by $f_{s}^{0}$, while that fraction going to generate energy is given by $f_{e}^{0}$ (Rittmann and McCarty, 2001). The sum of $f_{s}^{0}$ and $f_{e}^{0}$ is 1 . The fraction $f_{s}^{0}$ can also be converted into mass units to give a true cellular yield

$$
\Upsilon=\frac{f_{s}^{0}}{n_{e}}
$$

where $n_{e}$ is the number of electron equivalents in a mole of cells. The growth rate of cells can then be written as

$$
\frac{d B}{d t}=\Upsilon\left(\frac{-d C}{d t}\right)-b B
$$

where $\frac{d B}{d t}$ is the net growth rate (mass of cells per unit volume per time) of an active organism $B$, $\frac{-d S}{d t}$ is the rate of usage of the substrate, $b$ is the decay rate, and $\Upsilon$ is the true yield. Rittmann and McCarty (2001) have proposed a method for calculating the fraction of electrons going to cellular synthesis versus energy generation. Their approach should be included in the HPC for the cases where the yield must be calculated dynamically. 


\section{Colloid Transport Processes}

\subsection{Overview}

Colloids are very fine particles (such as clay minerals, metal oxides, viruses, bacteria, and organic macromolecules) that range in size between 1 and 10,000 nm (McCarthy and Zachara, 1989) and have high specific surface areas $(\sim 300 \mathrm{~m} 2 / \mathrm{g})$. The generation and mobilization of colloids are considered important issues in contaminant transport, particularly in the transport of radioactive true (intrinsic) colloids (e.g., colloidal $\mathrm{Pu}(\mathrm{IV})$ and $\mathrm{Pu}(\mathrm{V})$ ) and colloid-assisted transport of radioactive species (e.g., ${ }^{239} \mathrm{Pu},{ }^{237} \mathrm{~Np},{ }^{243} \mathrm{Am}$, and ${ }^{247} \mathrm{Cm}$ from high-level radionuclide wastes, or ${ }^{137} \mathrm{Cs},{ }^{90} \mathrm{Sr}$, and ${ }^{60} \mathrm{Co}$ from low-level radioactive wastes; see Meijer (2001) for sorption on pseudocolloids (e.g., naturally occurring clay colloids).

The DOE EM complex includes a diversity of hydrologic settings that involve transport of colloids. Such transport can be a cause of concern when contaminants (such as radionuclides) have sorbed on these colloids, or the colloids themselves have formed from supersaturation of contaminants. In the case of heavy metals and radionuclides, colloids are responsible for significant transport from the point of release, which is orders of magnitude larger than what would be expected if solute transport were the main mechanism of contaminant migration. Such transport has been observed at several locations that are part of the US DOE complex, and which represent a wide spectrum of geological and climatic conditions: LANL, INEL, LLNL, PNNL (Hanford site), Savannah River, Rocky Flats, Nevada Test Site etc. Additionally, concerns about colloid transport were significant drivers behind the analysis involved in the evaluation of the suitability of the Yucca Mountain site as the location for a proposed High level Nuclear Waster Repository.

The complexity of colloid-related transport necessitates inclusion of all the known processes (as described in the next sections). Therefore, it may be necessary to account for different colloid types involved in the transport of a particular species, or for subsets (each representing a range of the particle size distributions) of similar types of colloid colloids. In some of the more complex problems involving the transport of multidisperse colloids, it is entirely possible that up to 8 equations ( 2 for the main mass components, i.e., gas and water, one for a species of interest (e.g., $\mathrm{PuO}_{2}$ ), and up to five components representing distinct ranges of colloidal sizes) may need to be solved simultaneously. If colloidal and contaminant concentrations are low (i.e., unaffecting the water density), and/or if the flow fields become time-invariant within a relatively short time, it is possible that the flow and transport equations can be decoupled and solved separately, thus speeding up execution. Thus, the code capabilities need to be flexible, accommodating the entire range from very demanding situations necessitating the solution of the fully-coupled strongly-nonlinear problem, to more easily solved scenarios in which linearization is possible and flow and transport can be safely decoupled.

It is envisaged that the software describing colloid transport will be accessible by all flow-related software developed within the framework of the ASCEM. However, it is expected that two-phase flow systems (involving the flow of water and air) in saturated-unsaturated geologic media will represent the vast majority of the problems to be encountered, i.e., it is not expected that there will be a need to involve 3-phase flow physics. Additionally, in cases in which the gas phase is not expected to exhibit significant pressure changes, it is expected that a single flow equation (i.e., 
the Richards' equation, rather than the two full equations for the air-water system) will suffice to describe the hydrologic regime that controls colloid transport.

\subsubsection{Colloidal Behavior}

Radioactive true colloids or radionuclides adsorbed onto pseudocolloids can be transported farther than free radioactive elements (McCarthy and Zachara, 1989). The significant migration of strongly sorbing $\mathrm{Pu}$ and Am (more than $30 \mathrm{~m}$ ), from a low-level nuclear waste site at Los Alamos National Laboratory through unsaturated tuff over a period of approximately 30 years, is attributed to colloid and/or colloid assisted transport, a hypothesis confirmed by laboratory experiments (Buddemeier and Hunt, 1988). Using the ${ }^{240} \mathrm{Pu} /{ }^{239} \mathrm{Pu}$ isotope ratio to fingerprint the source of $\mathrm{Pu}$ in the water table, Kersting et al. (1999) demonstrated that soluble (ionic) Pu is practically immobile in the subsurface of the Nevada Test Site because of its strong sorption, but can be transported over significant distances (1.3 km over a 30 -year period) in colloidal form.

A complete description of colloid-facilitated radionuclide transport requires consideration of a large number of processes (EPRI, 1999), including advection, diffusion, colloid generation, colloid stability, colloid-solute-matrix interactions, affinity of colloids for the gas-water interface, colloid filtration (surface and straining), and kinetically controlled physical-chemical filtration.

\subsubsection{Colloid Types and Classes}

The analysis in Buck et al. (2003) identifies the following types of colloids (Figure 3), based on their origin and characteristics:

1. True (or intrinsic) colloids are generated from a solute when its concentration exceeds its solubility (Saltelli and Bidoglio, 1984). For example, true $\mathrm{Pu}(\mathrm{IV})$ colloids have been produced by the agglomeration of hydrolyzed Pu(IV) ions under acidic conditions (EPRI, 1999). When immature, actinide true colloids display hydrophilic properties, but become hydrophobic with increasing age.

2. Waste-form colloids are formed from the nucleation of colloids from waste form dissolution, and from spallation of colloid-sized waste from alteration products.

3. Pseudocolloids are all other colloidal particles, i.e., natural colloids that can be inorganic (e.g., clay, iron oxyhydroxides, silica) or organic (microbes and humic acids (Ibaraki and Sudicky, 1995). Pseudocolloids become carriers of contaminants (including radionuclides) when the corresponding solutes sorb onto them. (Note that in Buck et al. (2003) only radioactive pseudocolloids are referred to as such, while non-radioactive ones are referred to as seepage/groundwater colloids).

In terms of the mathematical description of their radioactive and transport behavior, these colloids are classified as follows: 
Class I. In this class the entire non-aqueous component of the colloidal particle is radioactive. True colloids and waste-form colloids are Class I colloids (Figure 3). It is not generally known whether, in radioactive Class I colloids, decay-induced recoil is sufficiently strong to cause ejection of daughters and shrinkage of the colloidal particle, whether the colloidal size is maintained but its density is reduced after daughter ejection, or whether the daughters remain trapped within the colloidal structure. If there is ejection, then either the colloid density or the colloid size decreases (leading to larger diffusion).

Class II. In Class II, only a portion of the colloidal particle (usually a very small one) involves a contaminant (e.g., is radioactive). This class includes radioactive pseudocolloids (Figure 3), in which the actinide is irreversibly sorbed onto the colloid or incorporated into the colloidal structure (e.g., through ion exchange). In this case, the actinide remains confined onto the colloid and does not exchange mass with its surroundings (i.e., the liquid phase or adjacent colloids). If the time of actinide production is the same (a valid approximation, especially in radionuclides with long half lives), then the colloidal particle concentration in the liquid (mobile colloids) and solid phases (immobile colloids, filtered or strained), and the radionuclide concentration of each colloid, are independent of each other and can be computed separately. Radioactive decay does not change the dimensions of Class II colloids because, even in the event of daughter ejection due to recoil, the actinide mass represents a very small portion of the total colloid mass, and the colloidal structure is maintained. Compared to the transport of Class I colloids of the same size with decaying sources, Class II colloids result in lower radioactivity concentrations (because actinides are a small portion of the total mass), but their relative concentrations (with respect to that at the release points) are the same.

Class III. Class III includes radioactive pseudocolloids in which actinides are reversibly sorbed onto the underlying natural colloid (Figure 3). As in Class II colloids, only a small portion of the colloidal particle is radioactive. In this case, the actinide components are not confined onto the colloid, but can exchange mass with their surroundings (i.e., the liquid phase or adjacent colloids). For the same reasons discussed in Class II colloids, radioactive decay does not change the dimensions of Class III colloids. However, determination of the radioactivity concentration is much more complex in Class III colloids than in Class I and II colloids (the transport equations of which can be linearized), because the corresponding equations are nonlinear.

Class IV. A fourth class of colloids, Class IV colloids, includes all nonradioactive colloids and can belong to any colloid type (true colloids or pseudocolloids).

\subsubsection{Colloid Generation and Stability}

The formation of mobile colloidal suspensions in the subsurface is attributed to a number of mechanisms:

1. matrix dissolution caused by changes in $\mathrm{pH}$ or redox conditions;

2. supersaturation with respect to the inorganic species; 

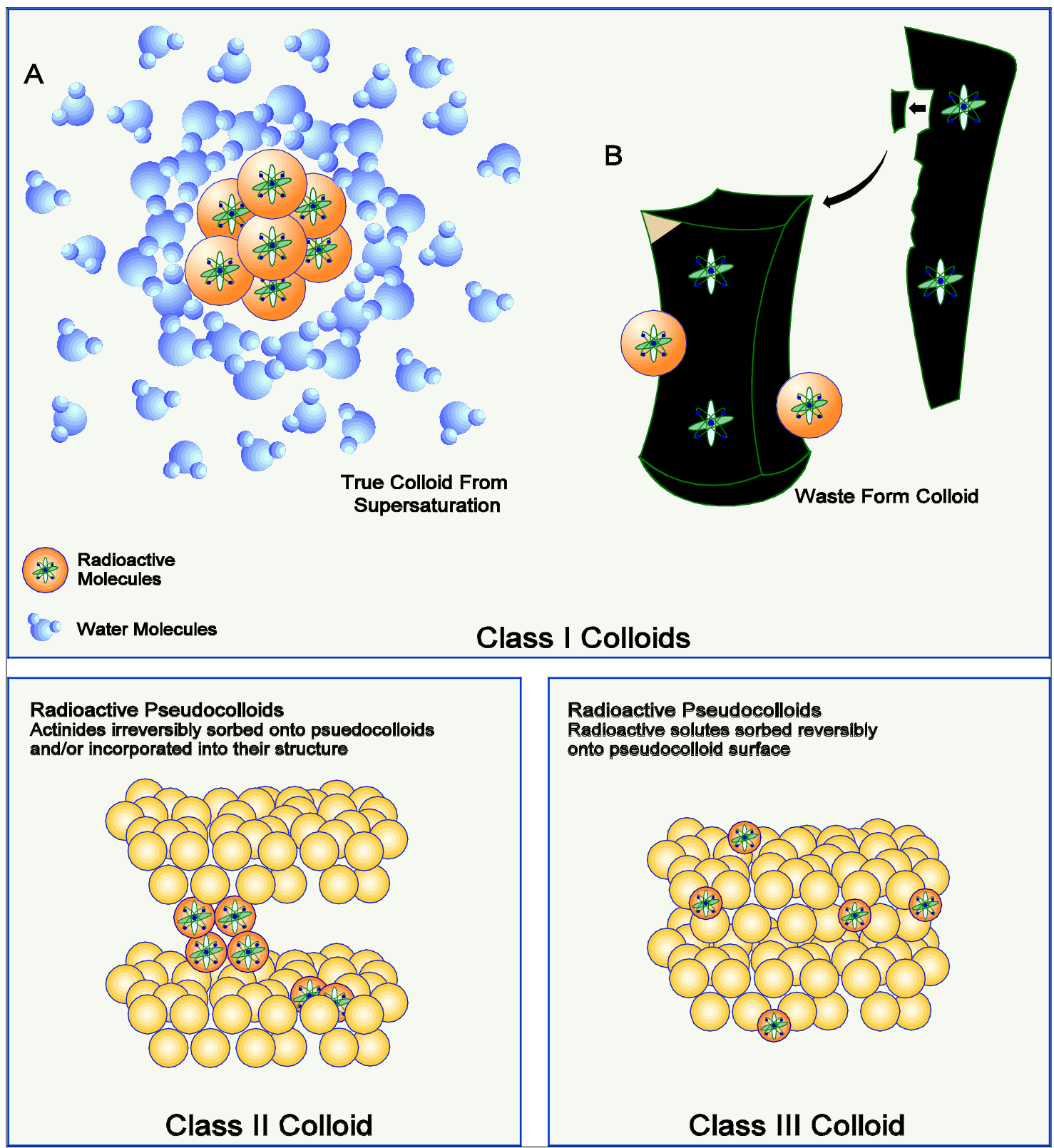

\section{Radioactive Pseudocollolds \\ Radloactive solutes sorbed reversibly \\ onto pseudocolloid surface}

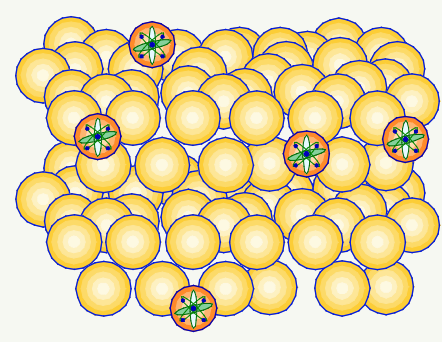

Class III Colloid

Figure 3: A conceptual image of the colloid classes I-III is presented along with their origins. 
3. disruption of the mineral matrix by large changes in the flow regimes resulting from injection, pumping, or large episodic rainfall infiltrations;

4. release and movement of viruses and bacteria; and

5. formation of micelles from the agglomeration of humic acids (Abdel-Salam and Chrysikopoulos, 1995).

6. Buddemeier and Hunt (1988) indicate that submicrometer colloids can easily be released from mineral and glass surfaces that are chemically, hydrodynamically, or mechanically stressed.

Colloid stabilization/destabilization includes steric stabilization by mechanisms such as organic coating of inorganic colloids, and the effects of $\mathrm{pH}$ and ionic strength on coagulation and precipitation. The stability of colloid suspension is very sensitive to changes in ionic strength (EPRI, 1999).

\subsubsection{Colloid Deposition}

Colloid deposition (physical-chemical filtration) during saturated flow through a porous medium is commonly assumed to occur in two steps: (1) transport of colloids to matrix surfaces by Brownian diffusion, interception, or gravitational sedimentation (i.e., colloid-matrix collision) and (2) attachment of colloids to matrix surfaces. The attachment efficiency (i.e., the fraction of collisions resulting in attachment) is strongly influenced by interparticle forces between colloids and matrix surfaces, such as van der Waals and electric double-layer interactions, steric stabilization, and hydrodynamic forces (Kretzschmar et al., 1995). Kretzschmar et al. (1997) demonstrated that colloid deposition generally follows a first-order kinetic-rate law and experimentally determined the corresponding collision efficiencies.

\subsubsection{Colloid-Contaminant-Matrix Interactions}

Colloid attachment to the host rock is strongly dependent on electrostatic interactions. Once attached, colloid detachment (declogging) is generally slow to irreversible (Ibaraki and Sudicky, 1995). Sorption of radionuclides on colloids is controlled by a range of chemical processes such as ion exchange, surface complexation, and organic complexation (Ding et al., 2006; EPRI, 1999).

If sorption of radioactive ions onto colloids is assumed to follow an equilibrium isotherm, radionuclides are stripped very quickly from the colloids when these enter a clean (radionuclide-free) part of the porous medium. This approach was incapable of explaining the long transport distances observed in field experiments (Van de Weerd and Leijnse, 1997). In the study, the problem was addressed by assuming (a) kinetic sorption of radionuclides onto the humic colloids and (b) kinetic colloid deposition (Van de Weerd and Leijnse, 1997). The sorption of dissolved ionic Pu(IV) onto hematite, goethite, montmorillonite, and silica colloids in both natural and carbonate-rich synthetic waters was reported to be fast (Meijer, 2001). Under equilibrium conditions, the colloid distribution coefficient $K_{d}$ values of $\mathrm{Pu}(\mathrm{V})$ was about $100 \mathrm{~mL} / \mathrm{g}$ for hematite and montmorillonite 
colloids. These very large values indicate that iron oxide and clay colloids can significantly enhance the transport of ${ }^{239} \mathrm{Pu}$. Very large $K_{d}$ values of actinide sorption are also confirmed in Buck et al. (2003). The values are so large that they can support an approximation of treating such radioactive Class III colloids as Class II colloids.

\subsubsection{Colloid Straining Filtration}

Colloid straining filtration is a physical retardation process. Wan and Tokunaga (1997) distinguish two types of straining: conventional straining (if the colloid is larger than the pore throat diameter or the fracture aperture) and film straining (if the colloid is larger than the thickness of the adsorbed water film coating the grains of the rock).

Wan and Tokunaga (1997) developed a conceptual model to describe colloid transport in unsaturated media as a function of water saturation $S_{w}$. If the rock $S_{w}$ exceeds a critical saturation value $S_{c}$, colloids move through the system entirely within the aqueous phase. For $S_{w}<S_{c}$, colloids can only move in the thin film of water that lines the grain boundaries, and colloid transport through the water film depends on two parameters: the ratio of the colloid size to the film thickness, and the flow velocity. Temporal variations in $S_{w}$ in the subsurface profile and in the infiltration rate can lead to strongly nonlinear colloid mobility in the vicinity of $S_{c}$.

McGraw and Kaplan (1997) investigated the effect of colloid size (from 52 to $1900 \mathrm{~nm}$ ) and $S_{w}$ (from 6 to $100 \%$ ) on colloid transport through unsaturated media in Hanford sediments. They showed a very strong dependence of filtration on the colloid size under unsaturated conditions. At a volumetric water content of $6 \%$, (the expected water content in the Hanford vadose zone), colloid removal increased exponentially with colloid size. The decrease in colloid mobility at low volumetric water contents was attributed to resistance caused by friction (as the colloids were dragged along the sand grains). Colloid retardation increased as the ratio between the water film thickness and colloid diameter decrease.

Straining filtration is determined by the ratio $R d=d g / d p$, where $d_{g}$ is the diameter of the grains of the porous medium and $d_{p}$ is the suspended particle diameter. Based on the experimental data of Sakthivadivel (1969), $R_{d}=10$ leads to cake filtration, $10<R_{d} \leq 20$ corresponds to substantial straining filtration (permeability reductions by a factor of 7-15 and particles occupying $0.3 \phi$ ), and $R_{d}>20$ results in limited straining (only $2-5 \%$ of $\phi$ occupied by particles, and permeability reductions by $10-50 \%$ ). Herzig et al. (1970) indicated that little straining was expected when $R_{d}$ $>12$, and calculated that when $R_{d}=50$, only $0.053 \%$ of $\phi$ would be occupied by particles.

\subsubsection{Other Processes}

Colloid Diffusion. Colloids diffuse slower than dissolved species because of their larger size. For the largest colloids, diffusion is approximately three orders of magnitude slower than that for molecular species (Nuttall et al., 1991). For example, the diffusion coefficient $D_{0}$ of a $0.1 \mu \mathrm{m}$ colloid at $20^{\circ} \mathrm{C}$ is $4.29 \times 10^{-12} \mathrm{~m}^{2} / \mathrm{sec}$, while the $D_{0}$ of $\mathrm{Br}^{-}$is $2.08 \times 10^{-9} \mathrm{~m}^{2} / \mathrm{sec}$ (Cussler, 1997). 
Pore Exclusion. Using mercury porosimetry, Roberts and Lin (1997) determined that the average pore diameters of welded and densely welded tuff samples ranged between 19.7 and 53.1. These extremely small pores are certain to exclude a significant proportion of colloids. It is possible for colloids to accumulate on the fracture walls and thus clog the matrix pores open to the fracture. This can lead to reduction in the matrix permeability and in the colloid diffusion into the matrix. Pore exclusion is not expected to be significant in the fractures, and colloids can travel cover significant distances in the fractures (especially given the limited diffusion into the matrix).

Role of Air-Water Interface. The affinity of colloids for the air-water interface depends on their hydrophobicity and electrostatic charge. Hydrophilic colloids, such as mineral fragments, have a low affinity for the interface, in contrast to hydrophobic colloids (such as organic colloids and microbes). This affinity increases with the positive charge on the colloids (EPRI, 1999). The flow and saturation conditions in the subsurface determine whether this enhances or retards transport. Note that the potential impact of the air-water interfaces on colloid transport has not been quantified yet.

\subsection{Process Model Equations}

\subsubsection{General Mass Balance Equation}

The conservation of mass for a tracer is given by (Equation (2.12))

$$
\frac{\partial \phi \sum_{\alpha} s_{\alpha} \rho_{\alpha} Y_{i, \alpha}}{\partial t}=\nabla \cdot \boldsymbol{J}_{i}+\boldsymbol{Q}_{i}
$$

where $Y_{i}$ mass fraction of tracer $\mathrm{i}$ (solute or colloid), $\phi$ is the porosity, $s_{\alpha}$ is the saturation of phase $\alpha, \rho$ is the density of phase $\alpha, \boldsymbol{J}_{i}$ the total mass flux of tracer $i$ and $Q_{i}$ the mass rate of the source/sink of tracer $i$.

\subsubsection{Accumulation Terms}

The total accumulation of tracer $i, Y_{i}$, (solute or colloid) in a porous or fractured medium (PFM) is given by:

$$
Y_{i}= \begin{cases}Y_{i, \alpha}+Y_{i, \Theta}+Y_{i, C} & \text { when } i \text { is a solute } \\ Y_{i, \alpha}+Y_{i, \xi} & \text { when } i \text { is a colloid }\end{cases}
$$

where $Y_{i, \alpha}$ is the mass fraction in phase $\alpha, Y_{i, \Theta}$ is the mass of tracer $i$ adsorbed onto the PFM grains or surface, $Y_{i, C}$ is the mass of tracer $i$ adsorbed onto pseudocolloidal particles, $Y_{i, \xi}$ is the mass of filtered colloidal tracer $i$.

Pseudocolloids are clearly differentiated from "true" colloids - i.e., those generated from contaminants when their concentrations exceed the solubility limit (Saltelli and Bidoglio, 1984) - and their transport behavior includes both transport of these colloids, as well as that of solute contaminants sorbed onto them (colloid-assisted transport). 
Accumulation of Filtered Colloids. Accumulation of filtered colloids is given by:

$$
Y_{i, \xi}=\rho_{C, i} \xi_{i}
$$

where $\rho_{C, i}$ is the density of colloidal tracer $i, \xi_{i}$ is the filtered concentration of colloids expressed as the volume of colloids per volume of the PFM.

For equilibrium filtration

$$
\xi_{i}=K_{\xi} \rho Y_{i}
$$

where $K_{\xi}$ is a distribution coefficient. Colloid filtration is more accurately described by a linear kinetic model (Corapcioglu et al., 1987), which can take the following form:

$$
\frac{\partial \xi_{i}}{\partial t}=k^{+} Y_{i}-k^{-} \xi_{i}
$$

where $k^{+}$and $k^{-}\left[s^{-1}\right]$ are the kinetic forward and reverse colloid deposition rates (clogging and declogging coefficients), respectively, which are specific to each colloid and rock type. The term $k^{-}$is commonly assumed to be zero (Bowen and Epstein, 1979), but there is insufficient evidence to support this assumption. An alternative is to set $k^{-}$equal to a fraction of $k^{+}$. Because the kinetic coefficient $k^{+}$is a linear function of the flow velocity, Equation (6.6), dependence of $k^{-}$on velocity appears to be conceptually sound.

From De Marsily (1986) and Ibaraki and Sudicky (1995), the following expression for the $k^{+}$ coefficient can be derived:

$$
k^{+}=\epsilon_{f} f_{v} q G_{B},
$$

where $\epsilon_{f}$ is the filter coefficient of the porous medium, $f_{v}$ is a velocity adjustment factor, $q$ is the Darcy velocity, and $G_{B}$ is a dynamic blocking function that describes the variation of the porosity and specific surface with $\xi_{i}$ (James and Chrysikopoulos, 1999). The factor $f(1 \leq f \leq 1.5)$ accounts for the velocity of the colloidal particle flow being larger than that of water (Ibaraki and Sudicky, 1995). This larger particle flow results from the relatively large size of the colloids, which tends to concentrate them in the middle of the pores where the water velocity is larger than the bulk average velocity. The factor $f_{v}$ tends to increase with decreasing ionic strength, but cannot exceed 1.5 because colloids cannot move faster than the maximum water velocity, which occurs at the middle of the pores and is equal to 1.5 times the average pore velocity (Ibaraki and Sudicky, 1995).

The filter coefficient $\epsilon$ can be computed from Harvey and Garabedian (1991) as

$$
\epsilon=1.5 \frac{1-\phi}{d_{m}} \alpha_{C} \eta_{C}
$$

where $d_{m}$ is the particle size of the medium grains or the fracture aperture $[L], \alpha_{C}$ is the collision efficiency factor, $\eta_{C}$ is the single collector efficiency that is given by

$$
\eta_{C}=0.9\left(\frac{k_{B} T}{\mu_{w} d_{c} d_{m} q}\right)^{2 / 3}+1.5\left(\frac{d_{c}}{d_{m}}\right)^{2}+\left(\rho_{C, i}-\rho\right) \frac{g d_{c}^{2}}{18 \mu_{w} q}
$$

where $k_{B}$ is the Boltzmann constant, $d_{c}$ is the colloid diameter $[m], T$ is the absolute temperature, $\mu_{w}$ is the water viscosity, and all other terms remain as previously defined. 
For deep filtration (i.e., in the case of very dilute colloidal suspensions), there is no interaction among the colloidal particles and no effects on the medium porosity $\phi$ and permeability, i.e., $\phi$ is constant, and $G=1$. Note that it is possible to have combined filtration, in which two different types of filtration (e.g., equilibrium and kinetic, or two kinetic filtrations with different $k^{+}$and $k^{-}$) occur simultaneously.

Accumulation of Solutes In Colloid-Assisted Transport. The mass of a tracer $i$ sorbed onto a set of pseudocolloids $j=1, \ldots, N_{C}$ is given by:

$$
Y_{\Theta, i}=\sum_{j=1}^{N_{C}}\left(\rho_{C, j} \xi_{j}+\rho Y_{j}\right) \Theta_{i, j}
$$

where $\Theta_{i, j}$ is the sorbed mass of solute $i$ per unit mass of the pseudocolloid $j\left[k g k g^{-1}\right.$, and $N_{C}$ is the total number of pseudocolloid species involved in the transport of the solute. The first term in the sum inside the parenthesis of Equation (6.9) describes the filtered (deposited) colloid concentration, and the second the concentration of the suspended colloids in the liquid phase. Note that Equation (6.9) applies to pseudocolloids only (Class III), as opposed to true colloids, onto which contaminants are not considered to sorb.

For equilibrium physical sorption,

$$
\Theta_{i}= \begin{cases}K_{d} \rho Y_{i} & \text { for linear equilibrium sorption, } \\ K_{F} \rho_{i}^{\beta_{F}} & \text { for Freundlich equilibrium sorption, } \\ \frac{K_{1} \rho Y_{i}}{1+K_{2} \rho Y_{i}} & \text { for Langmuir equilibrium sorption, }\end{cases}
$$

where $K_{d}\left[k^{-1} m^{3}\right], K_{F}\left[k g^{-\beta_{F}} m^{3 \beta_{F}}\right], K_{1}\left[k^{-1} m^{3}\right]$, and $K_{2}\left[k^{-1} m^{3}\right]$ are sorption parameters specific to each solute and rock type. Of particular interest is the parameter $K_{d}$, called the distribution coefficient, which is the constant slope of the linear equilibrium adsorption isotherm of a solute in relation to the medium. If a kinetic isotherm is followed, then sorption is described by:

$$
\frac{d \Theta_{i}}{d t}= \begin{cases}k_{l}\left(K_{d} \rho Y_{i}-\delta_{\Theta} \Theta_{i}\right) & \text { for linear kinetic sorption, } \\ k_{F}\left(K_{F}\left(\rho Y_{i}\right)^{\beta_{F}}-\Theta_{i}\right) & \text { for Freundlich kinetic sorption, } \\ k_{L}\left(\frac{K_{1} \rho Y_{i}}{1+K_{2} \rho Y_{i}}-\Theta_{i}\right) & \text { for Langmuir kinetic sorption, }\end{cases}
$$

where $\delta_{\Theta}$ is a Dirac delta function

$$
\delta_{\Theta}= \begin{cases}1 & \text { for reversible linear kinetics physical sorption } \\ 0 & \text { for irreversible linear kinetic physical sorption }\end{cases}
$$

and $k_{l}, k_{F}$ and $k_{L}$ are the kinetic constants for linear, Freundlich and Langmuir sorption, respectively $\left[s^{-1}\right]$. 


\subsubsection{Flux Terms}

The flux of tracer $i$ has contributions from advective, diffusive, and dispersive transport processes, and is given by

$$
\boldsymbol{J}_{i}=-\phi s_{A} \rho_{A} \boldsymbol{q} Y_{i}+\phi s_{A} \rho_{A} \boldsymbol{D}_{i}^{H} \nabla Y_{i}
$$

where $\boldsymbol{q}$ is the Darcy velocity vector of the aqueous phase, and $\boldsymbol{D}_{i}^{H}$ is the dispersion tensor of tracer $i$, a second order symmetric tensor with a principal axis aligned with the Darcy flow vector. Omitting the $i$ subscript, the dispersion tensor is described by the equations:

$$
\begin{gathered}
\boldsymbol{D}=D^{T} \boldsymbol{I}+\frac{D^{L}-D^{T}}{q^{2}} \boldsymbol{q q} \\
D^{L}=\phi s_{A} \tau D_{0}+\alpha_{L} q \\
D^{T}=\phi s_{A} \tau D_{0}+\alpha_{T} q
\end{gathered}
$$

where $\boldsymbol{I}$ is the unit vector, $\tau$ the tortuosity is coefficient of the pore paths $\left[\mathrm{m} \mathrm{m}^{-1}\right] ; D_{0}$ is the molecular diffusion coefficient of tracer $i$ in water; $\alpha_{L}$ and $\alpha_{T}$ are the longitudinal and transverse dispersivities, respectively $[m] ; \boldsymbol{q}$ is the Darcy velocity vector and $q$ is the magnitude of the velocity. Equations (6.13) through (6.16) apply to solutes, but need the following modifications to render them suitable for colloidal transport. More specifically:

1. The flux $\boldsymbol{J}_{\boldsymbol{A}}$ and the Darcy velocities $\boldsymbol{q}$ are multiplied by the factor $\phi$ (see Section 6.2.2).

2. The dispersivities $\alpha_{L}$ and $\alpha_{T}$ are generally different from those for solutes (Ibaraki and Sudicky, 1995) and may be a function of the colloidal particle size.

3. The term $D_{0}$ is the colloidal diffusion coefficient in water $\left[m^{2} s^{-1}\right]$ and is described by the Stokes-Einstein equation, according to Bird et al. (1960), as

$$
D_{0}=\frac{k_{B} T}{3 \pi \mu_{w} d_{p}},
$$

where $k_{B}$ is the Boltzmann constant $\left(1.38 \times 10^{-} 23 \mathrm{JK}^{-1}\right.$ in SI units), $T$ is the absolute water temperature, $\mu_{w}$ is the dynamic viscosity of water, and $d_{p}$ is the colloid diameter $[\mathrm{m}]$.

4. The fluxes in Equation (6.13) are multiplied by the colloid accessibility factors $f_{c}\left(0 \leq f_{c} \leq\right.$ 1 ) at the interface of different media. The $f_{c}$ factor describes the portion of the colloidal concentration in a medium that is allowed to enter an adjacent medium of different characteristics, and quantifies pore-size exclusion (straining).

In the treatment of the general 3-D dispersion tensor, velocities are averaged by using the projected area weighting method (Wu et al., 1996), in which a velocity component $q_{j}(j \equiv x, y, z)$ of the vector $\boldsymbol{q}$ is determined by vectorial summation of the components of all local connection vectors in the same direction, weighted by the projected area in that direction. This approach allows the solution of the transport problem in irregularly shaped grids, in which the velocities normal to the interface areas are not aligned with the principal axes. 


\subsubsection{Data Needs}

The flow problem that defines the transport problem has specific data needs that are addressed elsewhere, and will not be further discussed here. Data needs related to the colloid transport problem include the transport properties of (a) the porous/fractured media, (b) of the colloids and (c) of the solutes described in the earlier sections (including colloidal size range, accessibility factors, velocity factors, pore size distribution for straining evaluation, filtration parameters, sorption type and sorption properties of solute species onto colloids in cases of colloid-assisted solute transport, etc.). It is possible that some of these data needs can be covered from earlier laboratory studies, and from data in the literature. If no previous data exist, the use of analogs and extensive sensitivity analysis of the system behavior are a reasonable approach.

\subsubsection{Boundary Conditions, Sources and Sinks}

For colloid transport studies, the needed boundary conditions include (a) those of the hydrological system and (b) those of the transport problem. The former are addressed by the solution of the flow problem and will not be further discussed here. The latter are usually quite simple because they either describe releases, or indicate lack of contaminants at the outer boundaries (or even inner) of the simulated domain. There is significant uncertainty related to source/sink terms, as these are not always known or well constrained. Lack of sufficiently accurate information on the subject will probably necessitate significant sensitivity analysis of the impact of sources and sinks on colloidal transport.

\subsubsection{General and Other Considerations}

The general analysis presented in the earlier sections is general is scope, and can be applied without any limitations if the needed transport data are available. If, as is expected, the colloid-transport capability will be developed in a flexible manner that will allow user-defined levels of complexity, coupling and non-linearity, then no serious limitations of application are expected. However, it is important to note that, for colloid-assisted solute transport, the code will have to be coupled with solute transport capabilities (probably developed by other entities), or to include its own internal colloid-assisted transport capability.

Multi-scale Versions. It is expected that multi-scale methods (e.g., multi-porosity, multi-permeability, multiple interacting continua, etc.) will be necessary in some of the studies, especially when describing colloid transport through fractured media. The use of such methods is not expected to pose any fundamental difficulties, as the general colloid transport equations apply without any modification. However, such applications will necessitate knowledge of potential straining/filtration at the subdomain interfaces, as well as knowledge of the transport properties and characteristics of each subdomain. 


\section{Thermal Processes}

\subsection{Overview}

Heat flow and thermal conduction is an important aspect of many geochemical systems affecting chemical processes through changes in equilibrium and kinetic rate constants. Non-isothermal conditions can also result in buoyancy driven flow resulting in convection cells and causing fingering phenomena due to differences in density.

\subsection{Process Model Equations}

One form of the governing equation for energy conservation in a porous medium with porosity $\phi$ is given by

$$
\frac{\partial}{\partial t}\left[\phi \sum_{\alpha} s_{\alpha} \rho_{\alpha} U_{\alpha}+(1-\phi) \rho_{r} c_{r} T\right]+\nabla \cdot\left[\sum_{\alpha} \boldsymbol{q}_{\alpha} \rho_{\alpha} H_{\alpha}-\kappa \nabla T\right]=Q_{e}
$$

In this equation $T$ refers to temperature and the subscript $\alpha$ represents a fluid phase with Darcy velocity $\boldsymbol{q}_{\alpha}$, density $\rho_{a}$, saturation $s_{\alpha}$, internal energy $U_{\alpha}$, and enthalpy $H_{\alpha}$. The coefficient $\kappa$ denotes the thermal conductivity of the medium and $c_{r}$ and $\rho_{r}$ refer to the specific heat and density of the porous medium. The quantity $Q_{e}$ denotes a source/sink term. Internal energy and enthalpy are related by the equation

$$
U_{\alpha}=H_{\alpha}-\frac{p_{\alpha}}{\rho_{\alpha}}
$$

Thermal conductivity if often described by the phenomenological relation given by Somerton et al. (1974)

$$
\kappa=\kappa_{\mathrm{dry}}+\sqrt{s_{l}}\left(\kappa_{\mathrm{sat}}-\kappa_{\mathrm{dry}}\right),
$$

where $\kappa_{\text {dry }}$ and $\kappa_{\text {sat }}$ are dry and fully saturated rock thermal conductivities, and $s_{l}$ denotes the saturation state of $\mathrm{H}_{2} \mathrm{O}$.

\subsection{Data Needs}

Equations of state for fluid density, internal energy and/or enthalpy are needed in addition to heat capacity and thermal conductivity of the porous medium. Often the fluid properties for a complex mixture is unknown and the pure phase end member properties are used.

\subsection{Boundary Conditions, Sources and Sinks}

Boundary conditions may take the form of specified temperature or heat flux including zero temperature gradient. Initial conditions include specifying the temperature over the computational domain such as a constant value or derived from the geothermal gradient. 


\subsection{Coupling Considerations}

As heat flow is coupled to the Darcy flux, the heat equation itself is coupled to the flow equation as well as reactive transport equations through heat generated by chemical reactions. Conversely the flow and reactive transport equations are coupled to the heat equation through the temperature dependence of fluid properties such as density, viscosity, internal energy and enthalpy, and equilibrium thermodynamic and kinetic rate constants. 


\section{Geomechanical Processes}

\subsection{Overview}

We define geomechanical models in the broad sense of models that describe coupled processes that act to deform, or change the mechanical properties of the sediment, soil, or rock. Geomechanics can play a very important role in the fluid flow of large groundwater basins. Changes in earth stresses, from either natural (e.g. earthquakes) or anthropogenic (e.g. pumping) can affect porosity and permeability in aquifers. Similarly, in some EM applications various processes may cause significant changes in the stress field of the aquifer. For example, remediation treatments may inject large volumes of water at temperatures that are significantly different from the background temperature. In these cases a formal treatment of mechanics models may be required.

In addition to the mathematical/empirical description of geomechanical processes using constitutive equations (e.g. Hookes Law), we also can include the underlying mechanistic equations that may lead to a specific stress-strain relation. These may include coupled mechanical-chemical processes (e.g., swelling, pressure solution, subcritical crack growth) mediated by transport and stress. It also may include dissolution-precipitation leading to cementation (strengthening) or reactions leading to weakening (e.g., many silicate to clay weathering reactions).

This broad class of geomechanical models is targeted for development in later years, however, it is important to provide background information that may impact the current requirements and design documents. Thus, the following discussion is intended to provide a high-level overview of concepts, and to summarize key elements in the mathematical formulation.

\subsection{Assumptions and Applicability}

While some shallow aquifer applications may require soil plasticity to be considered for slope stability considerations, it is assumed that linear elasticity, Hookes law, will be sufficient for most EM applications. In addition, the speed of sound for the rock or soil matrix in a typical aquifer will be much faster than the sound speed in the fluid. Thus the force balance equations that represent subsurface stresses are essentially steady state. Additionally, the velocity of the rock deformation will be assumed instantaneous relative to the fluid velocity and not considered further.

\subsection{THM Formulation}

The momentum conservation equation for static geomechanical process is

$$
\boldsymbol{\nabla} \cdot \boldsymbol{\sigma}+\rho \boldsymbol{g}=0,
$$

where $\boldsymbol{\sigma}$ is the total stress tensor and $\boldsymbol{g}$ the gravitational acceleration vector. To describe the interaction between the flow and deformation, the concept of effective stress has been introduced by K.Terzaghi in 1923. This concept was further generalized by Maurice Biot in a number of papers (Biot, 1941, 1955, 1973; Biot and Willis, 1957). Extending the Terzaghi's effective stress to the unsaturated state, we obtain

$$
\boldsymbol{\sigma}=\boldsymbol{\sigma}^{\prime}-\alpha_{B} p \boldsymbol{I}
$$


where $p$ is the average pore pressure, $\boldsymbol{\sigma}^{\prime}$ the effective stress tensor, and $\alpha_{B}$ the Biot coefficient. Introducing the displacement vector $\boldsymbol{d}$, recalling the stress-strain relation, and taking into consideration the thermal expansion effect, the effective stress tensor can be written as

$$
\boldsymbol{\sigma}^{\prime}=\boldsymbol{C}: \boldsymbol{\epsilon}-K \beta_{T} T \boldsymbol{I}, \quad \boldsymbol{\epsilon}=\frac{1}{2}\left(\boldsymbol{\nabla} \boldsymbol{d}+(\boldsymbol{\nabla} \boldsymbol{d})^{T}\right) \equiv D(\boldsymbol{d}),
$$

where $\boldsymbol{C}$ is the fourth-order tangential elastic modulus tensor, $\boldsymbol{\epsilon}$ the strain tensor, $T$ the temperature, $K$ the drained bulk modulus of the medium, and $\beta_{T}$ the volumetric thermal expansion coefficient.

Note that the model for the effective stress can be extended, for example, by incorporating swelling due to moisture. Collecting the above, the momentum equation becomes

$$
\boldsymbol{\nabla} \cdot(\boldsymbol{C}: D(\boldsymbol{d}))-\boldsymbol{\nabla}\left(\alpha_{B} p+K \beta_{T} T\right)+\rho \boldsymbol{g}=0
$$

Thus, the flow equations affect the stresses through the temperature and pressure gradient terms in the above equation. Additional coupling of the flow and force balance equations occurs though the porosity and permeability terms in the flow equations. The displacements $\boldsymbol{d}$ calculated with the force balance equations are used to calculate volume strains and porosity changes and these changes are used to update permeabilities with some conceptual model.

\subsection{Single-Phase Biot Model}

The Biot model for a single phase flow is derived here under the following assumptions: (a) temperature of the reservoir is constant, (b) there is no mass exchange between the rock phase and the fluid phase, (c) the rock is elastic material, and (d) the fluid is incompressible. Two fundamental physical processes are the mass conservation and momentum balance for both fluid and rock phases.

The constitutive equation for the effective stress is

$$
\boldsymbol{C}: \boldsymbol{\epsilon}=\frac{E_{Y}}{1+\nu_{p}}\left(\boldsymbol{\epsilon}+\frac{\nu_{p}}{1-2 \nu_{p}} \operatorname{trace}(\boldsymbol{\epsilon}) \boldsymbol{I}\right),
$$

where $E_{Y}$ is Young's modulus of elasticity and $\nu_{p}$ Poisson's ratio. The governing equation for the rock deformation become:

$$
-\nabla \cdot\left[\mu_{L}\left(\boldsymbol{\nabla d}+(\boldsymbol{\nabla} \boldsymbol{d})^{T}\right)\right]-\nabla\left(\lambda_{L} \boldsymbol{\nabla} \cdot \boldsymbol{d}\right)+\nabla\left(\alpha_{B} p\right)=0,
$$

where $\mu_{L}$ and $\lambda_{L}$ are the Lamé constants:

$$
\lambda_{L}=\frac{E_{Y} \nu_{p}}{\left(1+\nu_{p}\right)\left(1-2 \nu_{p}\right)} \text { and } \mu_{L}=\frac{E_{Y}}{2\left(1+\nu_{p}\right)} .
$$

Note that $\mu_{L}$ is sometimes called the shear modulus of the rock.

Generally speaking, $\alpha_{B}$ could be a function of the fluid pressure and rock deformation. However, for the simplified model, it is reasonable to assume that $\alpha_{B}$ is the physical constant (Biot, 1941). 
To derive the second governing equation, we shall follow (Biot, 1941). It has been shown there that the fundamental relation describing the increment $\theta$ of fluid volume per unit volume of soil may be written as

$$
\theta=\alpha_{1} \boldsymbol{\nabla} \cdot \boldsymbol{d}+\alpha_{2} p,
$$

where $\alpha_{1}$ measures the ratio of the fluid volume squeezed out to the volume change of the soil if the latter is compressed while allowing the fluid to escape and $\alpha_{2}$ is a measure of the amount of fluid which can be forced into the soil under pressure while the volume of the soil is kept constant.

Since the fluid is incompressible, the mass conservation law states that the rate of fluid content in an element of soil must be equal to the volume of fluid entering per second through the surface of the element plus a flow rate $Q$ from an external source or sink:

$$
\frac{\partial \theta}{\partial t}=-\nabla \cdot \boldsymbol{q}+Q
$$

or

$$
\frac{\partial\left(\alpha_{1} \operatorname{div} \boldsymbol{d}\right)}{\partial t}+\frac{\partial\left(\alpha_{2} p\right)}{\partial t}+\nabla \cdot \boldsymbol{q}=Q
$$

The fluid flow equations are solved together with the force balance equation (8.2).

\subsection{Boundary Conditions}

Boundary conditions for the force balance equations consist of fixed displacements $\boldsymbol{d}$ at aquifer boundaries or a variety of stress conditions that replicate known (measured or inferred) earth stresses. 


\section{Source Terms}

\subsection{Cementitious Source Terms}

Cement waste forms and barriers are among the most important of the source terms that need to be considered in a comprehensive Environmental Management simulation tool. Engineered barriers including cementitious barriers are used at sites disposing or contaminated with low-level radioactive waste to enhance performance of the natural environment with respect to controlling the potential spread of contaminants. Drivers for using cementitious barriers include: high radionuclide inventory, radionuclide characteristics (e.g., long half-live, high mobility due to chemical form / speciation, waste matrix properties, shallow water table, and humid climate that provides water for leaching the waste).

The ASCEM integrated modeling and simulation effort will develop several possibilities for dealing with the cementitious source terms in the context of the ASCEM modeling and simulation tool:

1. Make use of the simulation tools developed by the Cementitious Barriers Partnership (CBP) to represent the cementitious source terms, including the release of contaminants and the collateral effects of cement chemistry (e.g., high $\mathrm{pH}$ ) on groundwater chemistry, or

2. Build on the conceptual models developed by the Cementitious Barriers Partnership to develop complementary capabilities using the software tools in the HPC.

With the first option, the understanding is that the code or codes developed by the Cementitious Barriers Partnership will be used as a source term, although possibilities for feedbacks between the processes modeled by the ASCEM software (e.g., flow) and those modeled by the CBP code (e.g., cement degradation) will be limited under this scenario. The Cementitious Barriers Partnership will be providing a detailed set of process models and their mathematical formulation for later versions of this document. Many of the equations for an ASCEM HPC treatment of the cement degradation and release processes are to be found throughout this document. Subsequent versions of this document will describe the coupled set of equations that need to be considered.

\subsection{Glass Waste Forms}

A mathematical formulation for glass waste form corrosion over laboratory and geological periods of time is under development as part of the Nuclear Energy Advanced Modeling and Simulation (NEAMS) effort within the Department of Energy. The goal of this effort is to develop one or more rate laws for glass corrosion that can be upscaled from the molecular to microscopic continuum to macroscopic continuum scales. The upscaled rate law will be used in the ASCEM HPC code. However, it is useful to discuss some of the key issues and processes associated with glass corrosion and these are presented in the following sections. 


\subsubsection{Short and Long-Term Corrosion Rates}

A large amount of information on the glass-water reaction collected over the past 25 years has been summarized in the "glass compendium" (Cunnane, 1994) and numerous reviews (Barkatt et al., 1986; Bourcier, 1991, 1994; Bunker et al., 1988; Casey and Bunker, 1990; Hench et al., 1986; Icenhower et al., 2004; McGrail et al., 1997; Strachan and Croak, 2000; Vernaz and Dussossoy, 1992; Vernaz et al., 2001; Werme et al., 1990). Based on these reviews, the glass dissolution reaction can be divided into four regimes or stages (Figure 1) that occur as the reaction proceeds (e.g., Stage I, II, III, and IV).

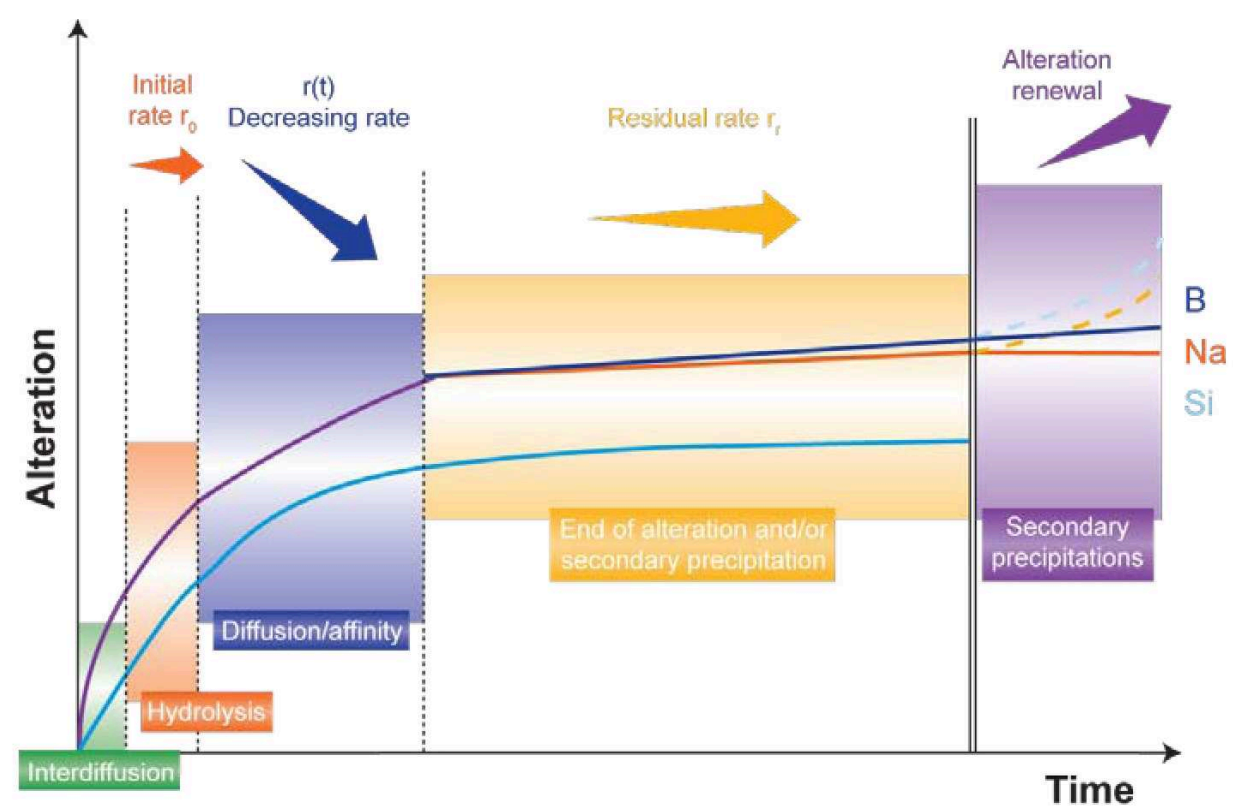

Figure 4: General schematic of the stages of glass-water reaction

Stage I, commonly referred to as the initial rate, consists of the following processes: upon initial contact by water, alkali cations are extracted by interdiffusion, which is considered a diffusioncontrolled reaction such that hydrogen or hydronium ions from the solution exchange for alkali ions contained in the glass. The process of interdiffusion is followed by two simultaneous reactions; hydration and dissolution of the glass network. In dilute solutions, the TST-based model successfully accounts for silicate dissolution in terms of temperature, $\mathrm{pH}$, and reactive surface area. As the concentration of dissolved components increases and the build-up of these components approach the formation of a thermodynamically unstable phase (hydrated surface layer), Stage II is reached.

During Stage II the matrix dissolution rate becomes dependent on the solution saturation state (concentration of elements in solution). Therefore, the process of ion exchange reaches a relatively constant rate in accordance with a diffusion-controlled process as a hydrated surface layer (e.g., gel layer) develops on the surface of the glass over time. The hydrated surface layer forms when relatively insoluble glass components (i.e., Al, Fe, and $\mathrm{Si}$ ) accumulate in the bulk solution and condense at the glass-water interface. Unlike the rate of ion-exchange, the dissolution rate of the 
glass network decreases because of the common ion effect (i.e., as the solution becomes more concentrated in glass components). The difference in chemical potential between the glass and aqueous phase decreases, which decreases the dissolution rate. This decrease in the rate of matrix dissolution is partially due to the effect $\mathrm{H}_{4} \mathrm{SiO}_{4}(\mathrm{aq})$ has on the dissolution rate and the formation of the hydrated surface layer (Abraitis et al., 2000; Pierce et al., 2004). In other words, as the activity of $\mathrm{H}_{4} \mathrm{SiO}_{4}(\mathrm{aq})$ increases in the aqueous solution the rate of glass dissolution decreases. It is important to note that in the case of glass, the dissolution rate cannot become zero because silicate glasses are thermodynamically unstable in water.

During Stage III the solution becomes saturated and secondary minerals begin to form, the alteration phase is often a simple clay mineral, such as a smectite or chlorite. The precipitation kinetics associated with these phases can be complex, but in general the rate of secondary phase growth increases in response to the increase in magnitude of supersaturation (Nagy, 1995; Nagy and Lasaga, 1993). Depending on the type of alteration phase, the glass-water reaction can increase from the residual rate and return to the forward rate (e.g., Stage IV - alteration rate renewal). This type of behavior has been observed in accelerated weathering experiments and may be associated with the $\mathrm{Al}:$ Fe ratio of the glass formulation (Jantzen et al., 2008).

\subsubsection{Glass Passivation}

It has been hypothesized that alteration "protective gel" layers are formed during the glass dissolution that act as a diffusion barrier for the glass constituents, in particular silicon.

In Van Iseghem, Gin, Grambow, McGrail, Strachan, and Wicks (Van Iseghem et al.), the alteration layer is defined as all glass altered by a dissolution reaction, where three types of sublayers are distinguished (Figure 2). The diffusion layer is the layer closest to the pristine glass. In this (thin) layer, high diffusion gradients of alkalis, hydrogen and boron exist. Otherwise the composition of this layer is similar to the pristine glass Zavoshy et al. (1985). If the solution flows sufficiently slow, the layer closest to the solution contains phases which have precipitated from solution. This layer is called the precipitated layer. The newly formed phases are called secondary phases. The layer between the diffusion layer and the precipitated layer is called the gel (layer). Here, the abundance and ordering of secondary phases increases with distance from the glass (Zavoshy et al., 1985). Most of the secondary phases have nucleated within the layers (Zavoshy et al., 1985) suggesting a local equilibrium caused by the reaction of the remaining glass components with the pore solution.

A recent paper by Jantzen et al. (2010) reviews the thermodynamic and structural approaches to the prediction of glass durability. Mechanistic modeling of glass durability including the slowing of the dissolution rate due to affinity and/or surface layer effects was first modeled by Grambow and Müller (2001) and is referred to as the GM2001 model. The GM2001 model combines the effect of glass hydration by water diffusion with ion exchange and affinity-controlled glass network corrosion. The slowing of dissolution due to the effect of a growing surface gel layer is represented by a mass transfer resistance for silica by this layer. At the interface between the glass and the gel layer, a different "gel layer" is assumed to be hydrated glass that allows the diffusion of $\mathrm{H} 2 \mathrm{O}$ in and boron and alkali atoms out of the glass. A 2003 modification of the GM2001 model, known as the GM2003 model (Van Iseghem, Gin, Grambow, McGrail, Strachan, and Wicks, Van Iseghem et al.) treats silica dissolution and silica diffusion through the gel separately from water diffusion 
and boundary conditions are specified at the gel/diffusion layer and the gel/solution interfaces. Introducing a boron/alkali diffusion coefficient, the BRAG model describes boron/alkali diffusion in the diffusion layer as well (Aertsens, 2007). Recently, the Glass Reactivity with Allowance for the Alteration Layer (GRAAL) model (Frugier et al., 2009, 2008) has been proposed, which is dependent on the composition and the passivating nature of the gel layer, called the passivating reactive interphase (PRI). The GRAAL model considers that water diffusion in the passivating reaction zone (the gel formed under saturation conditions) is a rate-limiting step in the overall glass dissolution kinetics. Moreover, this passivation zone is a soluble phase whose stability is directly dependent on the nature of the secondary phases likely to precipitate and on the solution renewal conditions.

An alternate approach to modeling the effect of glass alteration layers has been used at the Hanford Site (Pierce et al., 2004). Alteration products were modeled as a series of amorphous solids. Using the boron release data from the PCT experiments with LAWA44 glass, a reaction progress value was calculated as a function of test duration.

\subsubsection{Radionuclide Release}

Before the ILAW can be disposed, DOE must conduct a PA for IDF that describes the long-term impacts of the disposal facility on public health and environmental resources. One of the inputs to the PA is estimates of radionuclide release rates from the engineered portion of the disposal facility (source term). These estimates are expected to be based on chemical reactions that occur in the near-field and are controlled by the dissolution of the vitrified matrix. Therefore to provide credible estimates, a mechanistic understanding of the basic physical and geochemical processes that control glass dissolution and hence, radionuclide release, must be understood and incorporated into models in order to effectively simulate the glass-water reaction over the period of regulatory concern (approximately 10000 years). Neglecting glass composition, the dissolution rate is a function of temperature, $\mathrm{pH}$, and solution composition of the fluid contacting the glass. The temperature of the IDF is a known constant, $15^{\circ} \mathrm{C}$. However, both $\mathrm{pH}$ and composition of the fluid contacting the glass are variables that are affected by flow rate, reactions with other engineered materials, gas-water equilibria, secondary phase precipitation, alkali-ion exchange, and by dissolution of the glass itself. Consequently, glass dissolution rates will vary both in time and as a function of position in the disposal system. There is no physical constant such as a "leach rate" or radionuclide release rate parameter that can be assigned to the glass waste form in such a dynamic system.

A model based on empirical release behavior of the glass cannot provide feedback regarding the impacts of design options on the disposal system performance. Therefore, the source-term analysis requires the use of a reactive chemical transport modeling framework that takes into account the coupled effects of fluid flow and glass-water reactions on the chemistry of fluids percolating through the disposal facility. The fluid chemistry is coupled with kinetic rate equations that describe the response of the glass corrosion rate to changes in fluid composition in the disposal facility or repository, all computed as a function of time and space. These kinetic rate equations assume

1. the dependence of dissolution and precipitation rates on departure from equilibrium are based on arguments and assumptions of Transition State Theory (TST) and, 


\section{Process Model Mathematical Formulation Requirements}

2. the driving force for the transformation of unstable to stable silicate materials is governed principally by the magnitude of displacement from thermodynamic equilibrium.

To predict the long-term fate of glass in the subsurface over the period of regulatory concern, a mathematical model that describes glass reactivity is needed. Over the last few decades, a general rate equation has been fashioned to describe the dissolution of glass (and more ordered materials) into aqueous solution. The overall dissolution equation for glass may be quite complex, for example:

$$
\begin{aligned}
& \text { LAWABP } 1+4.42 \times 10^{-1} \mathrm{H}^{+}+1.89 \times 10^{-1} \mathrm{H}_{2} \mathrm{O} \rightarrow 1.36 \times 10^{-1} \mathrm{AlO}_{2}^{-} \\
&+1.84 \times 10^{-1} \mathrm{~B}(\mathrm{OH})_{3}(\mathrm{aq})+1.13 \times 10^{-2} \mathrm{Cl}^{-}+1.82 \times 10^{-4} \mathrm{CrO}_{4}^{2-} \\
&+1.46 \times 10^{-3} \mathrm{~F}^{-}+2.16 \times 10^{-2} \mathrm{Fe}(\mathrm{OH})_{3}(\mathrm{aq})+1.54 \times 10^{-7} \mathrm{IO}_{3}^{-} \\
&+3.23 \times 10^{-2} \mathrm{~K}^{+}+8.48 \times 10^{-3} \mathrm{La}^{3+}+1.71 \times 10^{-2} \mathrm{Mg}^{2+}+4.46 \times 10^{-1} \mathrm{Na}^{+} \\
&+7.79 \times 10^{-4} \mathrm{HPO}_{4}^{2-}+3.52 \times 10^{-8} \mathrm{PuO}_{2}\left(\mathrm{CO}_{3}\right)_{3}^{4-}+8.63 \times 10^{-4} \mathrm{SO}_{4}^{2-} \\
&+1.77 \times 10^{-8} \mathrm{SeO}_{4}^{2-}+4.82 \times 10^{-1} \mathrm{SiO}_{2}(\mathrm{aq})+6.59 \times 10^{-7} \mathrm{TcO}_{4}^{-} \\
&\left.+2.15 \times 10^{-2} \mathrm{Ti}^{-} \mathrm{OH}\right)_{4}(\mathrm{aq})+9.81 \times 10^{-7} \mathrm{UO}_{2}(\mathrm{OH})_{2}(\mathrm{aq}) \\
&+2.20 \times 10^{-2} \mathrm{Zn}^{2+}+2.94 \times 10^{-2} \mathrm{Zr}(\mathrm{OH})_{4}(\mathrm{aq}) .
\end{aligned}
$$

However, as described below, the equation is based upon the TST of chemical kinetics, in which the overall reaction rate is governed by the slowest elementary reaction. Elementary reactions have simple stoichiometry and can be combined as an overall reaction. In many cases, the elementary reactions can only be inferred. As an example of the elementary reaction, consider the dissolution of $\mathrm{SiO}_{2}$ polymorphs to form silicic acid:

$$
\mathrm{SiO}_{2}(s)+2 \mathrm{H}_{2} \mathrm{O} \leftrightarrow \mathrm{SiO}_{2} \cdot 2 \mathrm{H}_{2} \mathrm{O}^{\ddagger} \rightarrow \mathrm{H}_{4} \mathrm{SiO}_{4}(a q),
$$

in which $\mathrm{SiO}_{2} \cdot 2 \mathrm{H}_{2} \mathrm{O}^{\ddagger}$ represents an activated complex. Note that the reactants and the activated complex in Equation (9.2) are linked by a double-headed arrow symbolizing a reversible reaction. Equation (9.2) also illustrates that the TST formulation assumes the decay of the activated complex is an irreversible reaction.

Previous studies have established that the corrosion rate of silicate waste glasses is a complex process that depends strongly on temperature, $\mathrm{pH}$, and the chemical composition of the aqueous solution contacting the glass. When the aqueous solution is dilute, the glass dissolves at a characteristic forward rate that depends only on glass composition, temperature, and solution $\mathrm{pH}$ (McGrail et al., 1997). In static systems, or where the rates of mass transport by fluid flow are slow, dissolution releases glass components into the aqueous solution, and the concentration of these elements in the contacting fluid increases. The buildup of these dissolved components leads to slower glass corrosion rates as the contacting solution becomes more concentrated. As solution concentrations of dissolved elements continue to increase, solubility limits with respect to secondary phase(s) are reached, and these phases may begin to precipitate. Because silicate glasses are meta-stable solids, thermodynamics dictates that the glass will continue to dissolve or transform into more stable alteration phases. The key factor controlling long-term durability of waste glasses is the rate at which this processes proceeds.

The rate law that appears to best describe this overall dissolution behavior developed by Aagaard 
and Helgeson (1982) and applied to glass by Grambow (1985) is presented as follows:

$$
r_{i}=k_{o}^{+} v_{i} a_{H^{+}}^{\eta} \exp \left(\frac{E_{a}}{R T}\right)\left[1-\left(\frac{Q}{K_{g}}\right)^{\sigma}\right] \prod_{j} a_{j} .
$$

The chief virtue of Equation (9.3) is that it can be directly input into reaction-transport codes for predicting the long-term dissolution behavior of glass. Another benefit of Equation (9.3) is that it is solidly based on the TST of chemical kinetics, in which a series of reaction rates are governed by the slowest elementary reaction. Therefore, it is simply necessary to ascertain the "rate limiting" step in dissolution, rather than attempt to fully understand all of the possible reactions and kinetic pathways that can occur during the reaction of glass with aqueous solution. Because this ratelimiting step is an "elementary reaction," the stoichiometry of the reaction is typically simple and can easily be modeled.

In addition, test results with LAWABP1 glass (and most other ILAW glasses) show that it is susceptible to a secondary reaction mechanism, alkali ion exchange, which can have a significant impact on solution $\mathrm{pH}$. This reaction results in the selective extraction of $\mathrm{Na}$ via a reaction

$$
\mathrm{LAWABP} 1 \mathrm{Na}+\mathrm{H}^{+} \rightarrow \mathrm{LAWABP} 1 \mathrm{H}+\mathrm{Na}^{+},
$$

where LAWABP1Na represents the unreacted glass containing $\mathrm{Na}$ and LAWABP1H represents a hydrated glass where the $\mathrm{Na}$ has been replaced with an equimolar amount of hydrogen.

\subsection{High-Level Radioactive Waste Tanks as Source Terms}

Historical releases of high-level radioactive waste from underground storage tanks at U.S. Department of Energy (DOE) sites were highly basic and high ionic strength, often with strong chelating agents present. Source terms for these release will require liquid release rates as well as the composition and speciation of the in-tank waste. Very few analyses of tank waste were performed; consequently, the compostion of the wastes is typically based on reconstructions of process waste streams and tank storage procedures. Physical and chemical characterization of tank sludge, solids, and interstitial liquids can be critical to the determination of the liquid components in a tank release. Prediction of the chemical behavior of the tank waste requires high-ionic strength thermodynamic models to accurately speciate the chemical components under the relatively extreme electrolyte, radioactivity, and temperature conditions. These predictive thermodynamic models are discussed in the section on Activity Corrections, (see Section 5.2).

In the simplest case, the tank waste source term would be concentrations of chemical species released at a liquid flow rate. Thermal effects from radiolytic heating could induce temperaturedependent fluid properties, reaction thermodynamics, and equations of state. Near-field analyses could include multiphase flow in the vadose zone immediately surrounding the tank. In the extreme case with self-boiling tanks, a heat pipe effect could set up where liquid water would evaporate, be driven away as water vapor before condensing at distance. The evaporation would result in hypersaline conditions with increasing liquid density and the precipitation of salts, which cause pore fluids to move toward the tank and lower the vapor pressure. 
In subsequent versions of this document, the complete set of coupled equations for treatment of the complex tank source terms will be presented. These conceptual and mathematical models, however, are currently still under development. 


\section{A Nomenclature}

\section{A.1 Variables}

The International System of Units (SI) are used as the basic units in Table 5 and Table 6. It is recommended that the internal implementation use a single consistent system of units. Any appropriate units can be used for the input database and output with appropriate conversion factors. Table 2, Table 3 and Table 7 contain additional variables specific to the Pitzer model, Section 5.2.5.

In the text, bold variables, $\boldsymbol{J}$, indicate vector or tensor quantities, while normal variables, $J$, indicate scalars.

Table 5: List of Latin variables. Unitless variables are indicated by -

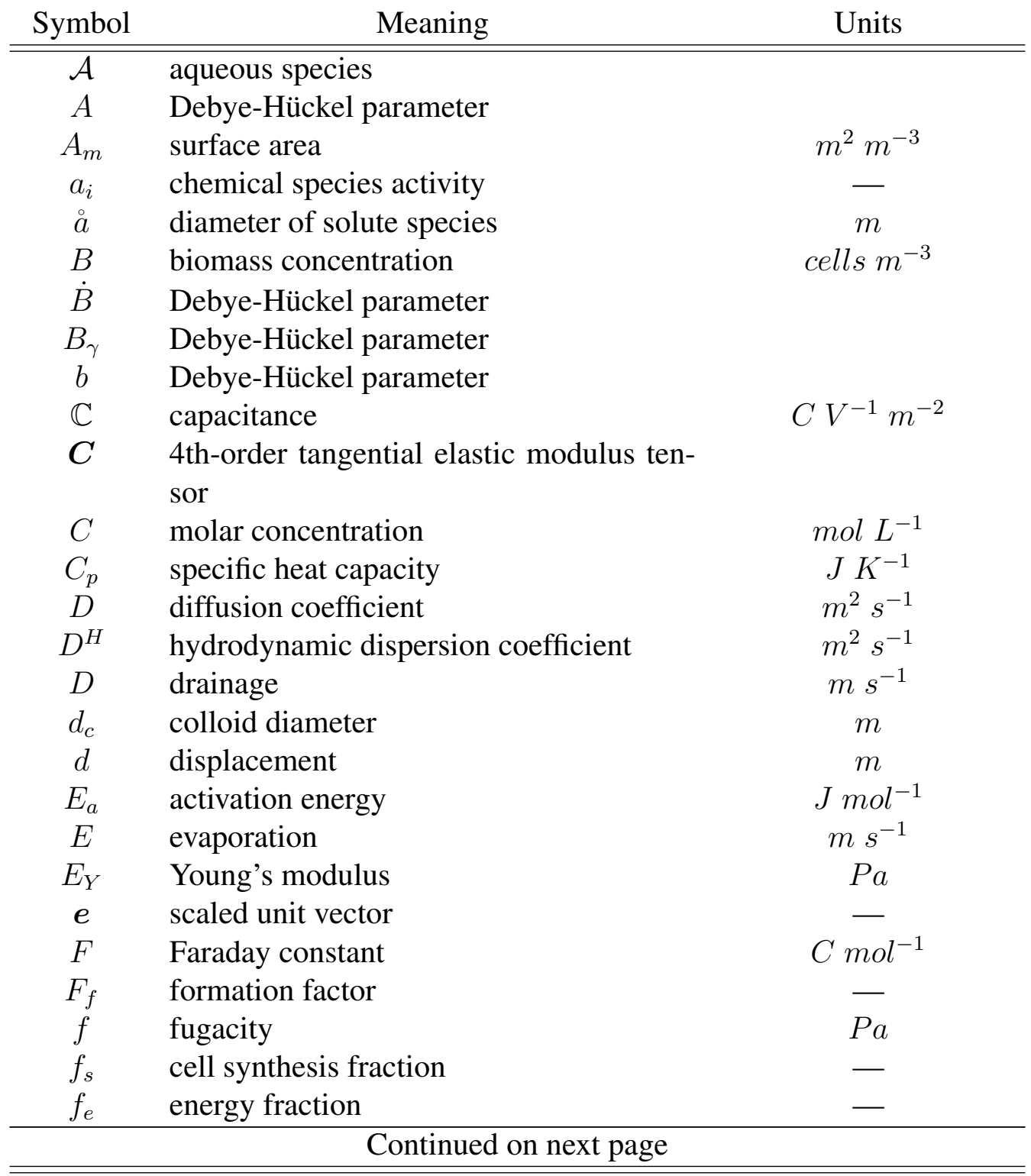


Table 5 - Continued

\begin{tabular}{|c|c|c|}
\hline Symbol & Meaning & Units \\
\hline$\overline{f_{v}}$ & velocity adjustment factor & - \\
\hline$f_{c}$ & colloid accessibility factor & - \\
\hline$G$ & Gibbs Energy & $J$ \\
\hline$G_{B}$ & dynamic blocking function & \\
\hline$G D$ & ground water recharge & $m s^{-1}$ \\
\hline$g$ & gravitational acceleration & $m s^{-2}$ \\
\hline$H$ & enthalpy & $J$ \\
\hline$h$ & hydraulic head & $m$ \\
\hline$h_{\alpha}$ & partial molar phase ethalpy & $J \mathrm{~mol}^{-1}$ \\
\hline$I$ & unit vector & \\
\hline$I^{i, j}$ & reaction rate & mol $m^{-3} s^{-1}$ \\
\hline$I$ & irrigation & $m s^{-1}$ \\
\hline$I$ & identity matrix & - \\
\hline $\bar{I}$ & ionic strength & mol $k g_{H_{2} O}^{-1}$ \\
\hline$i$ & current density & $A m^{-2}$ \\
\hline$J$ & flux & $k g m^{-2} s^{-1}$ \\
\hline$K$ & hydraulic conductivity & $m s^{-1}$ \\
\hline$K_{e q}, K_{m}$ & equilibrium constant & - \\
\hline$K_{i}$ & half saturation constant & mol $k g_{\mathrm{H}_{2} \mathrm{O}}^{-1}$ \\
\hline$K_{d}$ & distribution coefficient & $L^{3} k^{-1}$ \\
\hline$k$ & permeability & $m^{2}$ \\
\hline$k^{+}$ & forward rate constant & $s^{-1}$ \\
\hline$k^{-}$ & reverse rate constant & $s^{-1}$ \\
\hline$k_{B}$ & Boltzmann constant & $J K^{-1}$ \\
\hline$L_{i, j}$ & phenomenological coefficient & \\
\hline$L, L_{e}$ & path length & $m$ \\
\hline $\mathcal{M}$ & mineral species & - \\
\hline$M$ & mass & $\mathrm{kg}, \mathrm{mol}$ \\
\hline$M^{+}$ & competing cation & \\
\hline$m$ & molality & \\
\hline$N_{c}$ & number of components & - \\
\hline$N_{p}$ & number of phases & - \\
\hline$n$ & number of moles & mol \\
\hline$P$ & natural precipitation & $m s^{-1}$ \\
\hline$p$ & pressure & $P a$ \\
\hline$p_{c}$ & capillary pressure & $P a$ \\
\hline$Q$ & mass source or sink & $k g m^{-3} s^{-1}$ \\
\hline$Q_{T}$ & energy source/sink & $J m^{-3} s^{-1}$ \\
\hline$Q$ & ion activity product & - \\
\hline$q(i)$ & concentration of adsorbed cation & $\operatorname{mol} g^{-1}$ \\
\hline$q$ & Darcy flux & $m^{3} m^{-2} s^{-1}$ or $m s^{-1}$ \\
\hline
\end{tabular}




\begin{tabular}{|c|c|c|}
\hline Symbol & Meaning & Units \\
\hline $\bar{R}$ & Gas constant & $\overline{J J K^{-1} \mathrm{~mol}^{-1}}$ \\
\hline$R_{\text {over }}$ & overland flow & $m s^{-1}$ \\
\hline$R_{j}$ & rate of production of primary species & mol $m^{-3} s^{-1}$ \\
\hline$R_{j}$ & reaction rate & $\operatorname{mol~} m^{-3} s^{-1}$ \\
\hline$R_{H}$ & energy source term & $J m^{-3} s^{-1}$ \\
\hline$R_{f}$ & Retardation effect & - \\
\hline$r$ & radius & $m$ \\
\hline$S$ & entropy & $J K^{-1}$ \\
\hline$s$ & saturation & - \\
\hline$\Delta L_{f}$ & fracture spacing & $m$ \\
\hline$T$ & temperature & Kelvin \\
\hline$T_{t}$ & transpiration & $m s^{-1}$ \\
\hline$t$ & time & $s$ \\
\hline$t_{j}$ & transference number & - \\
\hline$U$ & internal energy & $J$ \\
\hline$u$ & internal energy & $J$ \\
\hline$u_{j}$ & species mobility & $m^{2}$ Volt $^{-1} s^{-1}$ \\
\hline$V$ & volume & $m^{3}$ \\
\hline$V_{m}$ & volume fraction & $m^{3} m^{-3}$ \\
\hline $\bar{V}$ & molar volume & $m^{3} \mathrm{~mol}^{-1}$ \\
\hline$v$ & fluid velocity & $m s^{-1}$ \\
\hline$W$ & formula or molecular weight & $\mathrm{kg} \mathrm{mol}^{-1}$ \\
\hline$W_{s t}$ & soil water storage & $m s^{-1}$ \\
\hline$X_{e x}$ & exchange site & mol $k g_{\mathrm{H}_{2} \mathrm{O}}^{-1}$ \\
\hline$X$ & mole fraction & - \\
\hline$Y$ & mass fraction & - \\
\hline$z_{i}$ & species charge & \\
\hline
\end{tabular}


Table 6: List of Greek variables. Unitless variables are indicated by -

\begin{tabular}{|c|c|c|}
\hline Symbol & Meaning & Units \\
\hline$\alpha_{L}$ & longitudinal dispersivity & $m$ \\
\hline$\alpha_{T}$ & transverse dispersivity & $m$ \\
\hline$\alpha_{B}$ & Biot coefficient & - \\
\hline$\beta$ & charge equivalent fraction & \\
\hline$\beta_{F}$ & Freundlich exponent & - \\
\hline$\beta_{T}$ & volumetric thermal expansion coefficient & \\
\hline$\gamma$ & activity coefficient & - \\
\hline$\Delta$ & film thickness & $m$ \\
\hline$\delta$ & aperture & $m$ \\
\hline$\epsilon$ & strain & - \\
\hline$\epsilon_{R}$ & relative dielectric constant & \\
\hline$\epsilon_{f}$ & filter coefficient & \\
\hline$\epsilon_{0}$ & permittivity of free space & $C^{2} J^{-1} m^{-1}$ \\
\hline$\eta$ & viscosity & $P a s^{-1}$ \\
\hline$\eta_{C}$ & single collector efficiency & \\
\hline$\Theta$ & angle & radians \\
\hline$\Theta_{i, j}$ & sorbent per unit mass pseudocolloid & $k g k g-1$ \\
\hline$\kappa$ & inverse of double layer thickness & $m^{-1}$ \\
\hline$\kappa_{e}$ & electrical conductivity & \\
\hline$\kappa_{T}$ & thermal conductivity & $W K^{-1} m^{-1}$ \\
\hline$\lambda$ & phase mobility & \\
\hline$\lambda_{w}$ & rational activity coefficient & - \\
\hline$\lambda_{L}$ & Lamé constant & \\
\hline$\lambda$ & axis of symmetry & - \\
\hline$\mu$ & dynamic viscosity & $\operatorname{Pas}$ \\
\hline$\mu_{i}$ & chemical potential & $J m^{-1}$ \\
\hline$\mu_{L}$ & Lamé constant, shear modulus & \\
\hline$\nu_{i j}$ & stoichiometric reaction coefficient & - \\
\hline$\nu_{p}$ & Poisson's ratio & - \\
\hline$\xi$ & filtered concentration of colloids & $m^{3} m^{-3}$ \\
\hline$\pi$ & mathematical constant & - \\
\hline$\rho$ & mass density & $k g m^{-3}$ \\
\hline$\rho^{\prime}$ & molar density & mol $m^{-3}$ \\
\hline$\sigma$ & stress & $P a$ \\
\hline$\sigma_{\alpha}$ & partial molar entropy & $J K^{-1} \mathrm{~mol}^{-1}$ \\
\hline$\sigma_{c d}$ & surface charge density & $C m^{-2}$ \\
\hline$\tau$ & tortuosity & - \\
\hline$\Upsilon$ & cell yield & cells mol $_{\text {substrate }}^{-1}$ \\
\hline$v$ & rate of substrate utilization & $s^{-1}$ \\
\hline \multicolumn{3}{|c|}{ Continued on next page } \\
\hline
\end{tabular}




\begin{tabular}{|c|c|c|}
\hline Symbol & $\begin{array}{l}\text { Table } 6 \text { - Continued } \\
\text { Meaning }\end{array}$ & Units \\
\hline$\Phi$ & electrical potential & Volts or $J C^{-1}$ \\
\hline$\phi$ & porosity & - \\
\hline$\varphi$ & osmotic coefficient & \\
\hline$\chi$ & average stoichiometric coefficient & \\
\hline$\Psi$ & total concentration of primary species & molal \\
\hline$\psi$ & surface potential & $V$ \\
\hline$\Omega$ & total flux of primary species & mol $m^{-2} s^{-1}$ \\
\hline$\nabla$ & vector differential operator & - \\
\hline
\end{tabular}

Table 7: List of variables used in the Pitzer, UNIQUAC and. NEA SIT models, Section 5.2

\begin{tabular}{cll} 
Symbol & \multicolumn{1}{c}{ Meaning } & Units \\
\hline \hline$\alpha$ & Pitzer parameter \\
$\beta$ & Pitzer interaction coefficient \\
$\epsilon$ & NEA SIT second-order interaction coeffi- \\
& cient \\
$\theta$ & Pitzer interaction coefficient \\
& UNIQUAC parameter \\
$\lambda$ & Pitzer interaction coefficient \\
$\mu$ & Pitzer interaction coefficient \\
$\sigma$ & Pitzer interaction coefficient \\
$\psi$ & UNIQUAC parameter \\
& Pitzer interaction coefficient \\
$A$ & Pitzer parameter \\
& Pitzer temperature function \\
$a$ & Pitzer temperature constants \\
$b$ & Pitzer parameter \\
$f$ & rational activity coefficient, UNIQUAC & - \\
$J$ & Pitzer Chebyshev polynomial \\
$q$ & UNIQUAC surface area parameter \\
$r$ & UNIQUAC volume parameter \\
$u$ & UNIQUAC pair interaction parameter \\
$z$ & UNIQUAC coordination number \\
\hline \hline
\end{tabular}




\section{REFERENCES}

Aagaard, P. and H. Helgeson (1982). Thermodynamic and kinetic constraints on reaction rates among minerals and aqueous solutions; I, Theoretical considerations. American journal of Science 282(3), 237.

Abdel-Salam, A. and C. V. Chrysikopoulos (1995). Modeling of colloid and colloid-facilitated contaminant transport in a two-dimensional fracture with spatially variable aperture. Transport in porous media 20(3), 197-221.

Abraitis, P., F. Livens, J. Monteith, J. Small, D. Trivedi, D. Vaughan, and R. Wogelius (2000). The kinetics and mechanisms of simulated British Magnox waste glass dissolution as a function of $\mathrm{pH}$, silicic acid activity and time in low temperature aqueous systems. Applied Geochemistry 15(9), 1399-1416.

Aertsens, M. (2007). The BRAG and GM2003 models for glass dissolution. Scientific Basis for Nuclear Waste Management XXX 985, 117-122.

Appelo, C. and D. Postma (1993). Geochemistry, groundwater, and pollution. Balkema, Rotterdam.

Aris, R. (1965). Prolegomena to the rational analysis of systems of chemical reactions. Archive for rational mechanics and analysis 19(2), 81-99.

Arnold, T., T. Zorn, H. Zänker, G. Bernhard, and H. Nitsche (2001). Sorption behavior of U (VI) on phyllite: experiments and modeling. Journal of contaminant hydrology 47(2-4), 219-231.

Bäder, F. G. (1978). Analysis of double-substrate limited growth. Biotechnology and Bioengineering 20(2), 183-202.

Banfield, J., G. Tyson, E. Allen, and R. Whitaker (2005). The search for a molecular-level understanding of the processes that underpin the Earth's biogeochemical cycles. Reviews in mineralogy and geochemistry 59(1), 1.

Barenblatt, G., I. Zheltov, and I. Kochina (1960). Basic Concepts in the Theory of Homogeneous Liquids in Fissured Rocks. J. Appl. Math. Mech 24, 1286-1303.

Barkatt, A., B. Gibson, P. Macedo, C. Montrose, W. Sousanpour, A. Barkatt, M. Boroomand, V. Rogers, and M. Penafiel (1986). Mechanisms of defense waste glass dissolution. Nuclear Technology 73(2), 140-164.

Barnett, M., P. Jardine, and S. Brooks (2002). U (VI) adsorption to heterogeneous subsurface media: Application of a surface complexation model. Environ. Sci. Technol 36(5), 937-942.

Bates, R. G. (1964). Determination of $p H$. Wiley and sons, New York.

Bear, J. (1972a). Dynamics of fluid in porous media.

Bear, J. (1972b). Dynamics of fluid in porous media. 
Berner, R. (1977). Stoichiometric models for nutrient regeneration in anoxic sediments. Limnology and Oceanography 22(5), 781-786.

Biot, M. (1941). General theory of three-dimensional consolidation. J. Appl. Phys. 12, 155-164.

Biot, M. (1955). Theory of elasticity and consolidation for porous anisotropic solid. J. Appl. Phys. 26, 182-185.

Biot, M. (1973). Nonlinear and semilinear rheology of porous solids. J. Geophy. Res. 73, 49244937.

Biot, M. and D. Willis (1957). The elastic coefficients of the theory of consolidation. J. Appl. Mech. 24, 594-601.

Bird, R. B., W. E. Stewart, and E. N. Lightfoot (1960). Transport Phenomena. New York: John Wiley \& Sons.

Bond, D., J. Davis, and J. Zachara (2008). Uranium(VI) release from contaminated vadose zone sediments: Estimation of potential contributions from dissolution and desorption. In M. Barnett and D. Kent (Eds.), Adsorption of metals by geomedia II: variables, mechanisms, and model applications, pp. 153. Elsevier Science Ltd.

Bourcier, W. (1991). Overview of chemical modeling of nuclear waste glass dissolution. In Fall meeting of the Materials Research Society (MRS), Boston, MA (United States), 24 Nov-1 Dec 1990.

Bourcier, W. (1994). Critical review of glass performance modeling. Technical report, ANL-94/17, Argonne National Lab., IL (United States).

Bourg, I., G. Sposito, and A. Bourg (2008). Modeling the diffusion of Na+ in compacted watersaturated Na-bentonite as a function of pore water ionic strength. Applied Geochemistry 23(12), $3635-3641$.

Bouwer, H. (1991). Simple derivation of the retardation equation and application to preferential flow and macrodispersion. Ground Water 29(1), 41-46.

Bowen, B. D. and N. Epstein (1979). Fine particle deposition in smooth parallel-plate channels. Journal of colloid and interface science 72(1), 81-97.

Bowen, R. M. (1968). On the stoichiometry of chemically reacting materials. Archive for Rational Mechanics and Analysis 29(2), 114-124.

Brantferger, K. M. (1991). Development of a thermodynamically consistent, fully implicit, compositional, equation-of-state, steamflood simulator. Ph. D. thesis, The University of Texas at Austin.

Brantferger, K. M., G. A. Pope, and K. Sepehrnoori (1991). Development of a thermodynamically consistent, fully implicit, equation-of-state, compositional steamflood simulator. SPE 21253, 471-480. 
Brooks, R. and A. Corey (1964). Hydraulic properties of porous media.

Buck, E., R. Aguilar, S. Alcorn, and J. Cunnane (2003). Waste form and in-drift colloidsassociated radionuclide concentrations: Abstraction and summary. Technical Report MDLEBS-PA-000004 REV 00, Bechtel SAIC Company, Las Vegas, Nevada.

Buddemeier, R. W. and J. R. Hunt (1988). Transport of colloidal contaminants in groundwater: radionuclide migration at the Nevada Test Site. Applied Geochemistry 3(5), 535-548.

Bunker, B., D. Tallant, and T. Headley (1988). Structure of leached sodium borosilicate glass. Physics and Chemistry of Glasses 29(3), 106-20.

Burdine, N. (1953). Relative permeability calculations from pore-size distribution data. Trans. AIME 198(1), 71-78.

Casey, W. and B. Bunker (1990). Leaching of mineral and glass surfaces during dissolution. Reviews in Mineralogy and Geochemistry 23(1), 397.

Cernik, M., M. Borkovec, and J. Westall (1996). Affinity distribution description of competitive ion binding to heterogeneous materials. Langmuir 12(25), 6127-6137.

Clegg, S. L. and P. Brimblecombe (1990). Solubility of Volatile Electrolytes in Multicomponent Solutions with Atmospheric Applications. In Melchoir, D. C. and Bassett, R. L., editors, Chemical Modelling of Aqueous Systems II, Volume 416, pp. 58-73. American Chemical Society, Washington D. C.

Corapcioglu, M. Y., N. M. Abboud, and A. Haridas (1987). Governing equations for particle transport in porous media. In J. Bear and M. Y. Corapcioglu (Eds.), Proceedings of the NATO Advanced Study Institute on Fundamentals of Transport Phenomena in Porous Media", Newark, Delaware, USA, July 14-23, 1985, pp. 209-342.

Cunnane, J. C. (1994). High-level waste borosilicate glass: A compendium of corrosion characteristics.

Curtis, G., J. Davis, and D. Naftz (2006). Simulation of reactive transport of uranium (VI) in groundwater with variable chemical conditions. Water Resour. Res 42(4).

Curtis, G., P. Fox, M. Kohler, and J. Davis (2004). Comparison of in situ uranium $K_{d}$ values with a laboratory determined surface complexation model. Applied Geochemistry 19(10), 1643-1653.

Cussler, E. L. (1997). Diffusion: Mass transfer in fluid systems. New York: Cambridge University Press.

Dale, A., P. Regnier, N. Knab, B. Joergensen, and P. Van Cappellen (2008). Anaerobic oxidation of methane (AOM) in marine sediments from the Skagerrak (Denmark): II. Reaction-transport modeling. Geochimica et Cosmochimica Acta 72(12), 2880-2894.

Dale, A., P. Regnier, and P. Van Cappellen (2006). Bioenergetic controls on anaerobic oxidation of methane (AOM) in coastal marine sediments: A theoretical analysis. American Journal of Science 306(4), 246. 
Davies, C. W. (1962). Ion Association. Butterworths, London.

Davis, J., J. Coston, D. Kent, and C. Fuller (1998). Application of the surface complexation concept to complex mineral assemblages. Environ. Sci. Technol 32(19), 2820-2828.

Davis, J. and D. Kent (1990). Surface complexation modeling in aqueous geochemistry. In M. F. Hochella and A. F. White (Eds.), Mineral-Water Interface Geochemistry, Volume 23, pp. 177260. Mineral Soc America.

Davis, J., D. Meece, M. Kohler, and G. Curtis (2004). Approaches to surface complexation modeling of Uranium (VI) adsorption on aquifer sediments1. Geochimica et Cosmochimica Acta 68(18), 3621-3641.

De Marsily, G. (1986). Quantitative Hydrogeology: Groundwater Hydrology for Engineers. San Diego, California: Academic.

Denbigh, K. (1981). The principles of chemical equilibrium: with applications in chemistry and chemical engineering. Cambridge Univ Pr.

Ding, M., P. W. Reimus, W. Lukens, S. Chipera, and C. Scism (2006). Sorption characteristics of radionuclides on clays in yucca mountain alluvium. In IHLRWM 2006, April 30 - May 4 2006, Las Vegas, Nevada.

Dzombak, D. and F. Morel (1990). Surface complexation modeling: Hydrous ferric oxide. WileyInterscience.

EPRI (1999). Colloids in Saturated and Partially-Saturated Porous Media, Approaches to the Treatment of Colloids in Yucca Mountain Total System Performance Assessment. Technical Report EPRI TR-112135, Electric Power Research Institute, Palo Alto, California.

Felmy, A. R. and J. H. Weare (1986). The prediction of borate mineral equilibrium in natural waters: Application to searles lake, california. Geochimica et Cosmochimica Acta 50, 27712783.

Freeze, R. and J. Cherry (1979). Groundwater. Prentice Hall, Englewood Cliffs, New Jersey.

Frugier, P., T. Chave, S. Gin, and J. Lartigue (2009). Application of the GRAAL model to leaching experiments with SON68 nuclear glass in initially pure water. Journal of Nuclear Materials.

Frugier, P., S. Gin, Y. Minet, T. Chave, B. Bonin, N. Godon, J. Lartigue, P. Jollivet, A. Ayral, L. De Windt, et al. (2008). SON68 nuclear glass dissolution kinetics: Current state of knowledge and basis of the new GRAAL model. Journal of Nuclear Materials 380(1-3), 8-21.

Gonçalvès, J., P. Rousseau-Gueutin, and A. Revil (2007, December). Introducing interacting diffuse layers in tlm calculations: A reappraisal of the influence of the pore size on the swelling pressure and the osmotic efficiency of compacted bentonites. Journal of Colloid and Interface Science 316(1), 92-99.

Grambow, B. (1985). A general rate equation for nuclear waste glass corrosion. In Mat. Res. Soc. Symp. Proc, Volume 44, pp. 15-27. 
Grambow, B. and R. Müller (2001). First-order dissolution rate law and the role of surface layers in glass performance assessment. Journal of Nuclear Materials 298(1-2), 112-124.

Greenber, J. P. and N. Moller (1989). The prediction of mineral solubilities in natural waters: A chemical equilibrium model for the Na-K-Ca-H-Cl-SO $\mathrm{SO}_{4}-\mathrm{H}_{2} \mathrm{O}$ system to high concentration from 0 to $250^{\circ}$ c. Geochimica et Cosmochimica Acta 53, 2503-2518.

Harvey, R. W. and S. P. Garabedian (1991). Use of colloid filtration theory in modeling movement of bacteria through a contaminated sandy aquifer. Environmental science \& technology 25(1), $178-185$.

Harvie, C. E. (1981). Theoretical Investigations in geochemistry and atom scattering. Ph. D. thesis, University of California, San Diego, CA.

Harvie, C. E., N. Moller, and J. H. Weare (1984). The prediction of mineral solubilities in natural waters: the $\mathrm{Na}-\mathrm{K}-\mathrm{Mg}-\mathrm{Ca}-\mathrm{H}-\mathrm{Cl}-\mathrm{SO}_{4}-\mathrm{OH}-\mathrm{HCO}_{3}-\mathrm{CO}_{3}-\mathrm{CO}_{2}-\mathrm{H}_{2} \mathrm{O}$ system to high ionic strengths at $25^{\circ}$ c. Geochimica et Cosmochimica Acta 48, 723-751.

Hellmann, R. and D. Tisserand (2006). Dissolution kinetics as a function of the Gibbs free energy of reaction: An experimental study based on albite feldspar. Geochimica et Cosmochimica Acta 70(2), 364-383.

Hench, L., D. Clark, and A. Harker (1986). Review. Nuclear Waste Solids. J. Mater. Sci. 21(5), 1457-1478.

Herzig, J. P., D. M. Leclerc, and P. Goff (1970). Flow of suspensions through porous media: Application to deep filtration. Industrial \& Engineering Chemistry 62(5), 8-35.

Hoehler, T. (2004). Biological energy requirements as quantitative boundary conditions for life in the subsurface. Geobiology 2(4), 205-215.

Hooyman, G. J. (1961). On thermodynamic coupling of chemical reactions. Proceedings of the National Academy of Sciences of the United States of America 47(8), 1169.

Hyun, S., P. Fox, J. Davis, K. Campbell, K. Hayes, and P. Long (2009). Surface complexation modeling of U (VI) adsorption by aquifer sediments from a former mill tailings site at Rifle, Colorado. Environmental Science \& Technology, 5465-5478.

Ibaraki, M. and E. A. Sudicky (1995). Colloid-facilitated contaminant transport in discretely fractured porous media 1. Numerical formulation and sensitivity analysis. Water Resources Research 31(12), 2945-2960.

Icenhower, J., S. Samson, A. Luttge, and B. McGrail (2004). Towards a consistent rate law: glass corrosion kinetics near saturation. Geological Society London Special Publications 236(1), 579.

James, S. C. and C. V. Chrysikopoulos (1999). Transport of polydisperse colloid suspensions in a single fracture. Water Resources Research 35(3), 707-718.

Jantzen, C., K. Brown, and J. Pickett (2010). Durable Glass for Thousands of Years. International Journal of Applied Glass Science 1(1), 38-62. 
Jantzen, C., D. Kaplan, N. Bibler, D. Peeler, and M. John Plodinec (2008). Performance of a buried radioactive high level waste (HLW) glass after 24 years. Journal of Nuclear Materials 378(3), 244-256.

Jin, Q. and C. Bethke (2005). Predicting the rate of microbial respiration in geochemical environments. Geochimica et Cosmochimica Acta 69(5), 1133-1143.

Kang, Q., P. Lichtner, and D. Zhang (2006). Lattice Boltzmann pore-scale model for multicomponent reactive transport in porous media. J. Geophys. Res 111.

Kent, D., R. Abrams, J. Davis, J. Coston, and D. LeBlanc (2000). Modeling the influence of variable $\mathrm{pH}$ on the transport of zinc in a contaminated aquifer using semiempirical surface complexation models. Water resources research 36(12), 3411-3425.

Kent, D., J. Davis, J. Joye, and G. Curtis (2008). Influence of variable chemical conditions on EDTA-enhanced transport of metal ions in mildly acidic groundwater. Environmental Pollution 153(1), 44-52.

Kent, D., J. Wilkie, and J. Davis (2007). Modeling the movement of a pH perturbation and its impact on adsorbed zinc and phosphate in a wastewater-contaminated aquifer. Water Resources Research 43(7), W07440.

Kersting, A. B., D. W. Efurd, D. L. Finnegan, D. J. Rokop, D. K. Smith, and J. L. Thompson (1999). Migration of plutonium in ground water at the Nevada Test Site. Nature 397(6714), $56-59$.

Kirkner, D. J. and H. Reeves (1988). Multicomponent mass transport with homogeneous and heterogeneous chemical reactions: The effect of the chemistry on the choice of numerical algorithm, part 1. Theory. Water Resources Research 24, 1719-1729.

Kohler, M., G. Curtis, D. Kent, and J. Davis (1996). Experimental investigation and modeling of uranium (VI) transport under variable chemical conditions. Water Resources Research 32(12), 3539-3551.

Kohler, M., G. Curtis, D. Meece, and J. Davis (2004). Methods for estimating adsorbed uranium (VI) and distribution coefficients of contaminated sediments. Environ. Sci. Technol 38(1), 240247.

Kretzschmar, R., K. Barmettler, D. Grolimund, Y. D. Yan, M. Borkovec, and H. Sticher (1997). Experimental determination of colloid deposition rates and collision efficiencies in natural porous media. Water Resources Research 33(5), 1129-1137.

Kretzschmar, R., W. P. Robarge, and A. Amoozegar (1995). Influence of natural organic matter on colloid transport through saprolite. Water Resources Research 31(3), 435-445.

Kumar, A. (1986). Prediction of densities of concentrated brines by pitzer theory. Journal of Chemical and Engineering Data 31, 19-20. 
Land, C. (1968). Calculation of imbibition relative permeability for two-and three-phase flow from rock properties. Old SPE Journal 8(2), 149-156.

Landry, C., C. Koretsky, T. Lund, M. Schaller, and S. Das (2009). Surface complexation modeling of Co (II) adsorption on mixtures of hydrous ferric oxide, quartz and kaolinite. Geochimica et Cosmochimica Acta 73(13), 3723-3737.

Langmuir, D. (1997). Aqueous Environmental Geochemistry. Prentice Hall, New Jersey.

Lasaga, A. (1998). Kinetic theory in the earth sciences. Princeton Univ Pr.

Lasaga, A. C. (1981). Rate laws of chemical reactions. In A. C. Lasaga and R. J. Kirkpatrick (Eds.), Kinetics of Geochemical Processes, Reviews in Mineralogy and Geochemistry, pp. 135169. Mineral Soc America.

Lasaga, A. C. (1984). Chemical kinetics of water-rock interactions. J. Geophys. Res 89(B6), 4009-4025.

Leroy, P., A. Revil, S. Altmann, and C. Tournassat (2007). Modeling the composition of the pore water in a clay-rock geological formation (Callovo-Oxfordian, France). Geochimica et Cosmochimica Acta 71(5), 1087-1097.

Li, L., C. Steefel, M. Kowalsky, A. Englert, and S. Hubbard (2009). Effects of physical and geochemical heterogeneities on mineral transformation and biomass accumulation during biostimulation experiments at Rifle, Colorado. Journal of Contaminant Hydrology.

Li, L., C. Steefel, and L. Yang (2008). Scale dependence of mineral dissolution rates within single pores and fractures. Geochimica et Cosmochimica Acta 72(2), 360-377.

Lichtner, P., S. Kelkar, and B. Robinson (2002). New form of dispersion tensor for axisymmetric porous media with implementation in particle tracking. Water Resources Research 38(8), 21-1 $-21-16$.

Lichtner, P. C. (1985). Continuum model for simultaneous chemical reactions and mass transport in hydrothermal systems. Geochimica et Cosmochimica Acta 49(3), 779-800.

Lorah, M. and L. Olsen (1999). Natural attenuation of chlorinated volatile organic compounds in a freshwater tidal wetland: Field evidence of anaerobic biodegradation. Water Resources Research 35(12).

Luckner, L., M. Van Genuchten, and D. Nielsen (1989). A consistent set of parametric models for the two-phase flow of immiscible fluids in the subsurface. Water Resources Research 25(10).

Maher, K., C. Steefel, A. White, and D. Stonestrom (2009). The role of reaction affinity and secondary minerals in regulating chemical weathering rates at the Santa Cruz Soil Chronosequence, California. Geochimica et Cosmochimica Acta 73(10), 2804-2831.

McCarthy, J. F. and J. M. Zachara (1989). Subsurface transport of contaminants. Environmental Science \& Technology 23(5), 496-502. 
McGrail, B., W. Ebert, A. Bakel, and D. Peeler (1997). Measurement of kinetic rate law parameters on a $\mathrm{Na}-\mathrm{Ca}-\mathrm{Al}$ borosilicate glass for low-activity waste. Journal of Nuclear Materials 249(23), 175-189.

McGraw, M. A. and D. I. Kaplan (1997). Colloid suspension stability and transport through unsaturated porous media. Technical Report PNNL-11565, Pacific Northwest National Laboratory, Richland, Washington.

Meijer, A. (2001). Unsaturated Zone and Saturated Zone Transport Properties (U0100). Technical Report AANL-NBS-HS-000019 REV 00 ICN 02, Bechtel SAIC Company, Las Vegas, Nevada.

Michelsen, M. and J. Mollerup (2004). Thermodynamic Models: Fundamentals \& Computational Aspects. Holte, Denmark: Tie-Line Publications.

Michelsen, M. L. (1999). State function based flash specifications. Fluid Phase Equilibria 158, 617-626.

Millington, R. and J. Quirk (1961). Permeability of porous solids. Transactions of the Farada Society 57, 1200-1207.

Moldrup, P., T. Olesen, P. Schjonning, T. Yamaguchi, and D. Rolston (2000). Predicting the gas diffusion coefficient in undisturbed soil from soil water characteristics. Soil Science Society of America Journal 64(1), 94.

Monnin, C. (1989). An ion interaction model for volumetric properties of natural waters: Density of the solution and partial molal volumes of electrolytes to higher concentrations at $25^{\circ} \mathrm{c}$. Geochimica et Cosmochimica Acta 53, 1177-1188.

Nagy, K. (1995). Dissolution and precipitation kinetics of sheet silicates. Reviews in Mineralogy 31, 173-234.

Nagy, K. and A. Lasaga (1993). Simultaneous precipitation kinetics of kaolinite and gibbsite at 80 $\mathrm{C}$ and $\mathrm{pH}$ 3. Geochimica et Cosmochimica Acta 57(17), 4329-4335.

Navarre-Sitchler, A., C. Steefel, L. Yang, L. Tomutsa, and S. Brantley (2009). Evolution of porosity and diffusivity associated with chemical weathering of a basalt clast. Journal of Geophysical Research-Earth Surface 114(F2), F02016.

Neuman, S. (1990). Universal scaling of hydraulic conductivities and dispersivities in geologic media. Water Resources Research 26(8), 1749-1758.

Newman, J. and K. Thomas-Alyea (2004). Electrochemical systems. Wiley-Interscience.

Nuttall, H. E., R. Jain, and Y. Fertelli (1991). Radiocolloid transport in saturated and unsaturated fractures. In High Level Radioactive Waste Management, Proceedings of the Second Annual International Conference, Las Vegas, Nevada, April 28-May 3, 1991, La Grange Park, Illinois, pp. 189-196. American Nuclear Society.

Onsager, L. (1931). Reciprocal Relations in Irreversible Processes. I. Physical Review 37(4), 405-426. 
Pabalan, R. and K. Pitzer (1987). Thermodynamics of concentrated electrolyte mixtures and the prediction of mineral solubilities to high temperatures for mixtures in the system $\mathrm{Na}-\mathrm{K}-\mathrm{Mg}-\mathrm{Cl}-$ SO4-OH-H2O. Geochimica et Cosmochimica Acta 51(9), 2429-2443.

Pape, H., C. Clauser, and J. Iffland (1999). Permeability prediction for reservoir sandstones and basement rocks based on fractal pore space geometry. Geophysics 64(5), 1447-1460.

Payne, T., J. Davis, M. Ochs, M. Olin, and C. Tweed (2004). Uranium adsorption on weathered schist-intercomparison of modelling approaches. Radiochimica Acta/International journal for chemical aspects of nuclear science and technology 92(9-11/2004), 651-661.

Pierce, E., B. McGrail, E. Rodriguez, H. Schaef, P. Saripalli, R. Serne, K. Krupka, P. Martin, S. Baum, K. Geiszler, et al. (2004). Waste Form Release Data Package for the 2005 Integrated Disposal Facility Performance Assessment. PNNL-14805, Pacific Northwest National Laboratory.

Pitzer, K. S. (1973). Thermodynamics of electrolytes - i. theoretical basis and general equations. Journal of Physical Chemistry 77, 268-277.

Pitzer, K. S. (1975). Thermodynamics of electrolytes - v. effects of higher-order electrostatic terms. Journal of Solution Chemistry 4, 249-265.

Pitzer, K. S. (1991). Ion interaction approach: Theory and Data Correlation in activity coefficients in Electrolyte Solutions. CRC Press, Boca Raton, Florida.

Pitzer, K. S. and J. M. Simonson (1986). Thermodynamis of multicomponent, miscible, ionic systems: theory and equations. Journal of Physical Chemistry 90, 3005-3009.

Plummer, L. N. and D. L. Parkhurst (1990). Application of the Pitzer Equations to the PHREEQE Geochemical Model. In Melchoir, D. C. and Bassett, R. L., editors, Chemical Modelling of Aqueous Systems II, Volume 416, pp. 58-73. American Chemical Society, Washington D. C.

Plummer, L. N., D. L. Parkhurst, G. W. Fleming, and S. A. Dunkle (1988). A computer program incorporating pitzer's equations for calculation of geochemical reactions in brines: United states geological survey water-resources investigations report. Technical Report 88-4153, USGS.

Prausnitz, J., T. Sherwood, and R. Reid (1977). The properties of gases and liquids. McGraw-Hill New York.

Prigogine, I. (1968). Introduction to Thermodynamics of Irreversible Processes. John Wiley \& Sons, Inc.

Pruess, K. and T. Narasimhan (1985). A practical method for modeling fluid and heat flow in fractured porous media. Soc. Pet. Eng. J 25(1), 14-26.

Reed, M. H. (1982). Calculation of multicomponent chemical equilibria and reaction processes in systems involving minerals, gases and an aqueous phase. Geochimica et Cosmochimica Acta 46(4), 513-528. 
Rittmann, B. and P. McCarty (2001). Environmental biotechnology: principles and applications. McGraw-Hill New York.

Roberts, J. J. and W. Lin (1997). Electrical properties of partially saturated Topopah Spring tuff: Water distribution as a function of saturation. Water resources research 33(4), 577-587.

Roden, E. (2008). Microbiological controls on geochemical kinetics. 1: Fundamentals and case study on microbial Fe (III) reduction. Kinetics of water-rock interactions. Springer, New York, NY 28, 335-415.

S. E. Drummond, J. (1981). Boiling and mixing of hydrothermal fluids: Chemical effects on mineral precipitation. Ph. D. thesis, The Pennsylvania State University, University Park, Pennsylvania.

Sakthivadivel, R. (1969). Clogging of a granular porous medium by sediment, Final Report. Technical Report Rep. HEL 15-7, Hydraulic Engineering Laboratory, College of Engineering, University of California, Berkeley, California.

Saltelli, A. and G. Bidoglio (1984). Americium filtration in glauconitic sand columns. NUCLEAR TECHNOL. 67(2), 245-254.

Schaap, M., F. Leij, and M. van Genuchten (2001). : a computer program for estimating soil hydraulic parameters with hierarchical pedotransfer functions. Journal of Hydrology 251(3-4), $163-176$.

Seitz, R., M. Freshley, B. Faybishenko, S. Hubbard, D. Watson, R. Roback, J. Blink, and A. Armstrong (2010). User suggestions for ascem requirements documents. Technical Report SRNLRP-2010-00856, Savannah River National Laboratory.

Slider, H. (1976). Practical Petroleum Reservoir Engineering Methods, An Energy Conservation Science. Petroleum Publishing Co., Tulsa, OK.

Somerton, W., A. El-Shaarani, and S. Mobarak (1974). High temperature behavior of rocks associated with geothermal type reservoirs. In SPE California Regional Meeting.

Spiteri, E. and R. Juanes (2006). Impact of relative permeability hysteresis on the numerical simulation of WAG injection. Journal of Petroleum Science and Engineering 50(2), 115-139.

Sposito, G. and D. Sparks (1981). The thermodynamics of soil solution. Oxford.

Steefel, C. and A. Lasaga (1994). A coupled model for transport of multiple chemical species and kinetic precipitation/dissolution reactions with applications to reactive flow in single phase hydrothermal systems. American Journal of Science 294(5), 529-592.

Steefel, C. and K. Maher (2009). Fluid-rock interaction: A reactive transport approach. Reviews in Mineralogy and Geochemistry 70(1), 485.

Steefel, C. and P. Van Cappellen (1990). A new kinetic approach to modeling water-rock interaction: The role of nucleation, precursors, and Ostwald ripening. Geochimica et Cosmochimica Acta 54(10), 2657-2677. 
Steefel, C. I. and K. T. B. MacQuarrie (1996). Approaches to modeling of reactive transport in porous media. In Reactive Transport in Porous Media, Reviews in Mineralogy and Geochemistry 34, pp. 83-125. Mineral Soc America.

Stone, H. (1973). Estimation of three phase relative permeability and residual oil data. J. Pet. Technol 12(4), 52-61.

Strachan, D. and T. Croak (2000). Compositional effects on long-term dissolution of borosilicate glass. Journal of Non-Crystalline Solids 272(1), 22-33.

Stumm, W. (1992). Chemistry of the solid-water interface: processes at the mineral-water and particle-water interface in natural systems. New York: John Wiley \& Sons.

Sumner, M. Handbook of soil science,. 2000.

Thomsen, K. (1997). Aqueous electrolytes: model parameters and process simulation.

Thomsen, K. (2005). Modeling electrolyte solutions with the extended universal quasichemical (UNIQUAC) model. Pure and applied chemistry 77(3), 531-542.

Van de Weerd, H. and A. Leijnse (1997). Assessment of the effect of kinetics on colloid facilitated radionuclide transport in porous media. Journal of contaminant hydrology 26(1-4), 245-256.

van Genuchten, M. (1980). A closed-form equation for predicting the hydraulic conductivity of unsaturated soils. Soil Sci. Soc. Am. J 44(5), 892-898.

Van Iseghem, P., S. Gin, B. Grambow, B. McGrail, D. Strachan, and G. Wicks. GLAMOR, A Critical Evaluation of the Dissolution Mechanisms of High Level Waste Glasses in Conditions of Relevance for Geological Disposal. Final report to the EC, in progress.

Van Zeggeren, F. and S. Storey (1970). The computation of chemical equilibria. Cambridge Univ Pr.

Vanselow, A. (1932). Equilibria of the base-exchange reactions of bentonites, permutites, soil colloids, and zeolites. Soil Science 33(2), 95.

Vaughan, P. (1989). Analysis of permeability reduction during flow of heated, aqueous fluid through westerly granite. In C. F. Tsang (Ed.), Coupled processes associated with nuclear waste repositories, pp. 529-539. Academic Press, New York.

Verma, A. and K. Pruess (1988). Thermohydrological conditions and silica redistribution near high-level nuclear wastes emplaced in saturated geological formations. J. Geophys. Res 93(B2).

Vernaz, E. and J. Dussossoy (1992). Current state of knowledge of nuclear waste glass corrosion mechanisms: the case of R7T7 glass. Applied Geochemistry 7, 13-22.

Vernaz, E., S. Gin, C. Jegou, and I. Ribet (2001). Present understanding of R7T7 glass alteration kinetics and their impact on long-term behavior modeling. Journal of Nuclear Materials 298(12), 27-36. 
Voegelin, A., V. Vulava, F. Kuhnen, and R. Kretzschmar (2000). Multicomponent transport of major cations predicted from binary adsorption experiments. Journal of contaminant hydrology 46(3-4), 319-338.

Wan, J. and T. K. Tokunaga (1997). Film straining of colloids in unsaturated porous media: Conceptual model and experimental testing. Environmental Science \& Technology 31(8), 24132420.

Ward, A. L. and G. W. Gee (1997). Performance evaluation of a field-scale surface barrier. Journal of Environmental Quality 26(3), 694.

Ward, A. L. and J. M. Keller (2005). Hydrology and vegetation data package for 200-uw-1 waste site engineered surface barrier design. Technical Report PNNL-15464, Pacific Northwest National Laboratory, Richland,WA.

Warren, J. and P. Root (1963). The behavior of naturally fractured reservoirs. Old SPE Journal 3(3), 245-255.

Werme, L., I. Bjorner, G. Bart, H. Zwicky, B. Grambow, W. Lutze, R. Ewing, and C. Magrabi (1990). Chemical corrosion of highly radioactive borosilicate nuclear waste glass under simulated repository conditions. Journal of Materials Research 5(5), 1130-46.

Wolery, T. J. (1969). Thermodynamics of hydrothermal systems at elevated temperatures and pressures. American Journal of Science 267, 792-804.

Wolery, T. J. (1992a). EQ3NR, A Computer Program for geochemical aqueous speciationsolubility calculations: Theoretical manual, Users Guide, and Related documentation (Version 7.0).

Wolery, T. J. (1992b). On the thermodynamic framework of solutions (with special refernce to aqueous electrolyte solutions. American Journal of Science 290, 296-320.

Wu, Y.-S., C. F. Ahlers, P. Fraser, A. Simmons, and K. Pruess (1996). Software qualification of selected TOUGH2 modules. Technical Report LBNL-39490, Lawrence Berkeley National Laboratory, Berkeley, California.

Yabusaki, S., Y. Fang, and S. Waichler (2008). Building conceptual models of field-scale uranium reactive transport in a dynamic vadose zone-aquifer-river system. Water Resources Research 44(W12403).

Yang, L. and C. Steefel (2008). Kaolinite dissolution and precipitation kinetics at $22 \mathrm{C}$ and pH 4. Geochimica et Cosmochimica Acta 72(1), 99-116.

Zavoshy, S., P. Chambre, and T. Pigford (1985). Mass transfer in a geologic environment. Proceedings of the Materials Research Society, Scientific Basis for Nuclear Waste Management VIII, 311-322.

Zhang, D. (2001). Stochastic Methods for Flow in Porous Media Coping with Uncertainties. Academic Press, London. 
Zhang, F., J. Parker, S. Brooks, Y. Kim, G. Tang, P. Jardine, and D. Watson (2009). Comparison of Approaches to Calibrate a Surface Complexation Model for U (VI) Sorption to Weathered Saprolite. Transport in Porous Media 78(2), 185-197.

Zyvoloski, G., B. Robinson, Z. Dash, and L. Trease (1997). Summary of the models and methods for the FEHM application-a finite-element heat-and mass-transfer code. Technical report, LA13307-MS, Los Alamos National Lab., NM (US).

Zyvoloski, G., B. Robinson, and H. Viswanathan (2008). Generalized dual porosity: A numerical method for representing spatially variable sub-grid scale processes. Advances in Water Resources 31(3), 535-544. 


\section{DISCLAIMER}

This document was prepared as an account of work sponsored by the United States Government. While this document is believed to contain correct information, neither the United States Government nor any agency thereof, nor The Regents of the University of California, nor any of their employees, makes any warranty, express or implied, or assumes any legal responsibility for the accuracy, completeness, or usefulness of any information, apparatus, product, or process disclosed, or represents that its use would not infringe privately owned rights. Reference herein to any specific commercial product, process, or service by its trade name, trademark, manufacturer, or otherwise, does not necessarily constitute or imply its endorsement, recommendation, or favoring by the United States Government or any agency thereof, or The Regents of the University of California. The views and opinions of authors expressed herein do not necessarily state or reflect those of the United States Government or any agency thereof or The Regents of the University of California.

Ernest Orlando Lawrence Berkeley National Laboratory is an equal opportunity employer. 